\title{
Estimating Production Functions with Control Functions When Capital Is Measured With Error
}

\author{
Kyoo il Kim* \\ University of Minnesota, Twin Cities \\ Amil Petrin \\ Suyong Song \\ University of Minnesota, Twin Cities and NBER University of Wisconsin, Milwaukee
}

December 2013

\begin{abstract}
We revisit the production function estimators of Olley and Pakes (1996) and Levinsohn and Petrin (2003). They use control functions to address the simultaneous determination of inputs and productivity. Both assume that input demand is a monotonic function of productivity holding capital constant and then invert this function to condition on productivity during estimation. If the observed capital variable is measured with error, investment will not generally be monotonic in the productivity shock holding observed capital constant. We develop consistent estimators of production function parameters in the face of this measurement error. Our identification and estimation results combine the nonlinear measurement error literature with Wooldridge (2005)'s joint estimation method to construct a proxy for productivity that addresses simultaneity. Our approach directly extends to the case where other inputs like intermediates or labor are observed with error.

Keywords: Production Function, Unobserved Productivity, Measurement Error, Nonparametric Estimation, Control Variate
\end{abstract}

JEL Classification: C14, C18, D24

\footnotetext{
${ }^{*}$ We thank Jinyong Hahn, Bruce Hansen, Yingyao Hu, Arthur Lewbel, Rosa Matzkin, and other seminar participants at UCLA, NSF-NBER CEME conference at Cornell University, 2013 North American Summer Meeting of Econometric Society at USC, IO conference at University of Tokyo, European Meeting of Econometric Society at Gothenburg, Midwest Econometrics Group at Bloomington for many helpful comments. Suyong Song gratefully acknowledges research grant from Yoshio Niho Excellence Fund. All errors are our own. Contacts: Kyoo il Kim at kyookim@umn.edu; Tel. 612-625-6793, Amil Petrin at petrin@umn.edu, and Suyong Song at songs@uwm.edu; Tel. 414-229-4283.
} 


\section{Introduction}

Production function estimates are a critical input into understanding the sources of economic growth. A major challenge in estimating production function parameters is that output and inputs are simultaneously determined (Marschak and Andrews (1944)). This simultaneity problem results in inputs being correlated with the productivity shock, leading to biased and inconsistent estimates of the parameters.

Historically researchers have used firm-specific fixed effects in panel data to address this problem, relying only on within firm variation in inputs over time to identify the parameters. In their review of the literature Grilliches and Mairesse (1998) remark that fixed effect estimators frequently have led researchers to find point estimates for the capital coefficient that are very low and often not significantly different from zero. They attribute these findings to capital being measured with a significant amount of error coupled with the fact that capital varies very little within firms over the time in a standard panel data set. The fixed effect effectively dispenses with all of the "signal," leading to a significant amount of attenuation bias in the capital coefficient.

A major contribution of Olley and Pakes (1996; hereafter OP) is their development of an estimation method that is both robust to the simultaneity problem and that does not dispense with all of the between-firm variation in capital. They assume that investment is a function of the capital and the productivity shock, the two state variables. Pakes (1996) provides conditions under which - for any given level of capital - investment is monotonically increasing in the productivity shock. By inverting the investment function they recover the productivity shock, on which they then condition during estimation. Unlike fixed effects the approach has the added advantage that the productivity shock is allowed to vary over time. Since its publication many researchers have confirmed that the OP approach generally leads to higher and more reasonable point estimates for capital than the fixed effect estimator.

One issue that has arisen for the OP estimator is that the inversion is only valid when investment is positive. Levinsohn and Petrin (2003; hereafter LP) note that in panel data many firms report zero investment, which can force researchers to drop a large fraction of their observations from the estimation procedure. ${ }^{1}$ They show that it is straightforward to extend the OP logic to intermediate inputs, writing intermediate demand as a function of the productivity shock and capital, and then inverting the intermediate demand function to proxy for the productivity shock.

While both methods add back a significant amount of variation in capital, neither method addresses the issue of measurement error in capital. Capital enters directly as a regressor in the production function and it is an argument in the control function used to condition out the productivity shock. The latter case is more difficult to address econometrically because capital enters the control function in an unknown non-linear way, making standard instrumental variable

\footnotetext{
${ }^{1}$ In the Chilean data they use investment is missing for between $30 \%$ and $70 \%$ of their observations depending upon which industry was under consideration. The fact that investment is lumpy in firm-level data also may suggest that investment does not fully respond to the productivity shock even when investment is positive.
} 
approaches inconsistent.

In this paper we show how to use recent insights from Hu and Schennach (2008) on nonclassical measurement error to allow for measurement error in capital and other inputs in the LP setting. To achieve identification we recast the conditional moment restrictions used in LP as integrals over conditional density functions of the true inputs. Using lagged input levels as instruments, Hu and Schennach's method can be used to estimate these conditional density functions when capital and possibly other inputs are measured with error. Once we have estimates of the conditional density functions of the true inputs we can integrate over them to calculate the LP conditional moment restrictions for any candidate parameter value. Our two-step estimator first uses sieve maximum likelihood (hereafter ML) estimation to estimate the conditional density functions. In the second step we use Ai and Chen (2003) to develop a sieve minimum distance (hereafter MD) estimator, integrating the conditional moment restrictions over the measurement-error-corrected conditional density estimates. ${ }^{2}$

The closest work to our paper is Huang and $\mathrm{Hu}$ (2011). They extend the LP setting to the case when intermediate inputs are measured with an additive error. We extend their approach to settings where both the intermediate input and capital can be contaminated by additive or non-additive measurement error.

The remainder of this paper is organized as follows. In Section 2 we review the OP and LP estimators and provide a non-technical overview of our estimation approach. In Section 3 we use the framework from Wooldridge (2005) to develop the general framework for our approach, including the case when both capital and intermediates are measured with error. Section 4 discusses identification and estimation and Sections 5 and 6 provide details on consistency, convergence rates, and asymptotic normality. Section 7 concludes and the technical details are gathered in the Appendix.

\section{Allowing for Measurement Error}

We review the OP and LP methodologies within the Wooldridge (2005) framework and show where measurement error in capital causes problems. We then show how to use recent results from the nonclassical measurement error literature to correct the problem.

\subsection{OP/LP Methodology}

The production function is written with the log of output as a function of the log of inputs and shocks

$$
y_{t}=\beta_{l} l_{t}+\beta_{k} k_{t}+\beta_{m} m_{t}+\omega_{t}+\epsilon_{t}
$$

where $l_{t}$ denotes labor, $k_{t}$ denotes capital, and $m_{t}$ denotes the intermediate input (such as materials or energy). $\omega_{t}$ is the productivity shock, a state variable observed by the firm but unobserved to

\footnotetext{
${ }^{2}$ This step extends $\mathrm{Ai}$ and Chen (2003) to a setting with unobserved endogenous variables.
} 
the econometrician and assumed to be a first-order Markov. $\omega_{t}$ is the source of the simultaneity problem as freely variable inputs $l_{t}$ and $m_{t}$ respond to it. $k_{t}$ is a state variable and is allowed to be correlated with $E\left[\omega_{t} \mid \omega_{t-1}\right]$, but it is assumed that $\xi_{t}=\omega_{t}-E\left[\omega_{t} \mid \omega_{t-1}\right]$, the innovation in the productivity shock, is uncorrelated with $k_{t}$. $\epsilon_{t}$ denotes an i.i.d. shock that is assumed to be uncorrelated with all of the inputs.

LP write intermediate input demand as a function of the state variables

$$
m_{t}=\mathbf{m}_{t}\left(\omega_{t}, k_{t}\right)
$$

and provide weak conditions under which $\mathbf{m}_{t}(\cdot, \cdot)$ is strictly monotonic in $\omega_{t}$ holding $k_{t}$ constant. The intermediate demand function can then be inverted to obtain the control function for $\omega_{t}$ as a function of observed $m_{t}$ and $k_{t}$, written as $\omega_{t}=h_{t}\left(m_{t}, k_{t}\right) .{ }^{3}$ Wooldridge (2005) uses a single index restriction to approximate unobserved productivity, so in the LP setting one has

$$
\omega_{t}=h_{t}\left(m_{t}, k_{t}\right)=\mathbf{c}\left(m_{t}, k_{t}\right)^{\prime} \beta_{\omega}
$$

where $\mathbf{c}\left(m_{t}, k_{t}\right)$ is a known vector function of $\left(m_{t}, k_{t}\right)$ chosen by researchers. He also writes the nonparametric conditional mean function $E\left[\omega_{t} \mid \omega_{t-1}\right]$ as

$$
E\left[\omega_{t} \mid \omega_{t-1}\right]=q\left(\mathbf{c}\left(m_{t-1}, k_{t-1}\right)^{\prime} \beta_{\omega}\right)
$$

for some unknown function $q(\cdot) .{ }^{4}$

Rewriting the production function as

$$
y_{t}=\beta_{l} l_{t}+\beta_{k} k_{t}+\beta_{m} m_{t}+E\left[\omega_{t} \mid \omega_{t-1}\right]+\xi_{t}+\epsilon_{t}
$$

yields

$$
\left[\xi_{t}+\epsilon_{t}\right](\theta)=y_{t}-\beta_{l} l_{t}-\beta_{k} k_{t}-\beta_{m} m_{t}-q\left(\mathbf{c}\left(m_{t-1}, k_{t-1}\right)^{\prime} \beta_{\omega}\right)
$$

with $\beta=\left(\beta_{l}, \beta_{k}, \beta_{m}, \beta_{\omega}\right), \theta=(\beta, q)$. Let the set of conditioning variables be $x_{t}=\left(k_{t}, k_{t-1}, m_{t-1}, l_{t-1}\right)$ and let $\theta_{0}$ denote the true parameter value. Wooldridge shows that the conditional moment restriction

$$
g\left(x_{t} ; \theta\right) \equiv E\left[\left[\xi_{t}+\epsilon_{t}\right](\theta) \mid x_{t}\right] \text { and } g\left(x_{t} ; \theta_{0}\right)=0
$$

is sufficient for identification of $\left(\beta_{l}, \beta_{k}, \beta_{m}\right)$ and $E\left[\omega_{t} \mid \omega_{t-1}\right] .{ }^{5}$ It is also robust to the Ackerberg,

\footnotetext{
${ }^{3} \mathrm{OP}$ write investment as a function of the two state variables $i_{t}=\mathbf{i}_{t}\left(\omega_{t}, k_{t}\right)$ and Pakes (1996) provides conditions under which investment is strictly monotonic in $\omega_{t}$ holding $k_{t}$ constant. OP then invert this function to get the control function with arguments $i_{t}$ and $k_{t}$.

${ }^{4} \mathrm{OP}$ use $i_{t}$ and $i_{t-1}$ instead of $m_{t}$ and $m_{t-1}$, respectively for $\omega_{t}$ and $E\left[\omega_{t} \mid \omega_{t-1}\right]$.

${ }^{5}$ In Section 3 we show how to identify $q$ and $\beta_{\omega}$ using an additional moment condition from LP.
} 
Caves, and Frazer (2006) criticism of LP (and OP). In equation (1) a function of $m_{t-1}$ and $k_{t-1}$ conditions out $E\left[\omega_{t} \mid \omega_{t-1}\right]$. $\xi_{t}$ is not correlated with $k_{t}$, so $k_{t}$ can serve as an instrument for itself. Lagged labor $l_{t-1}$ and lagged materials $m_{t-1}$ serve as instruments for $l_{t}$ and $m_{t} .{ }^{6}$ It is also clear from this moment why measurement error in $k_{t}$ is problematic for the LP (and OP) estimators as both current and lagged values of capital are used in the estimation, and the lagged values enter the estimation problem in a nonlinear way.

\subsection{Solution Overview}

Our approach is based on rewriting the estimation problem in terms of the conditional densities upon which it is based. For $g\left(x_{t} ; \theta\right)$ we have:

$$
\begin{aligned}
g\left(x_{t} ; \theta\right) \equiv & \int y_{t} f_{y_{t} \mid x_{t}} d y_{t}-\beta_{l} \int l_{t} f_{l_{t} \mid x_{t}} d l_{t}-\beta_{k} k_{t} \\
& -\beta_{m} \int m_{t} f_{m_{t} \mid x_{t}} d m_{t}-q\left(\mathbf{c}\left(m_{t-1}, k_{t-1}\right)^{\prime} \beta_{\omega}\right)
\end{aligned}
$$

and for the population objective function we have e.g.,

$$
\begin{aligned}
Q(\theta) & =E\left[g\left(x_{t} ; \theta\right)^{2}\right] \\
& =\int g\left(x_{t} ; \theta\right)^{2} f_{k_{t}, k_{t-1} \mid m_{t-1}, l_{t-1}} f_{m_{t-1}, l_{t-1}} d x_{t}
\end{aligned}
$$

(using Bayes Rule). Written in this way the OP/LP estimation problem can be viewed as one that requires estimates of the conditional densities $f_{y_{t} \mid x_{t}}, f_{l_{t} \mid x_{t}}$, and $f_{m_{t} \mid x_{t}}$ to estimate $g\left(x_{t} ; \theta\right)$ and then estimates of $f_{k_{t}, k_{t-1} \mid m_{t-1}, l_{t-1}}$ and $f_{m_{t-1}, l_{t-1}}$ to integrate to get the sample analog of $Q(\theta)$. Letting capital that is measured with error be denoted as $k_{t}^{*}$, and $x_{t}^{*}=\left(k_{t}^{*}, k_{t-1}^{*}, m_{t-1}, l_{t-1}\right)$, our main challenge is recovering the true densities given that the observed densities are $f_{y_{t} \mid x_{t}^{*}}, f_{l_{t} \mid x_{t}^{*}}$, and $f_{m_{t} \mid x_{t}^{*}}$ and $f_{k_{t}^{*}, k_{t-1}^{*} \mid m_{t-1}, l_{t-1}}\left(f_{m_{t-1}, l_{t-1}}\right.$ is directly observable from the data).

If we use $\left(k_{t-2}^{*}, k_{t-3}^{*}\right)$ as instruments for the current and lagged mismeasured capital measurements $\left(k_{t}^{*}, k_{t-1}^{*}\right)$, the true conditional densities can be recovered using recent developments in the nonclassical measurement error literature. In the data we observe the conditional density functions $f_{r_{t}, k_{t}^{*}, k_{t-1}^{*} \mid k_{t-2}^{*}, k_{t-3}^{*}, \mathbf{s}_{t}}$ for either (i) $r_{t}=y_{t}$ and $\mathbf{s}_{t}=\left(m_{t}, l_{t}, m_{t-1}, l_{t-1}\right)$ or (ii) $r_{t} \in\left\{l_{t}, m_{t}\right\}$ and $\mathbf{s}_{t}=\left(m_{t-1}, l_{t-1}\right)$. We can express these observed densities $f_{r_{t}, k_{t}^{*}, k_{t-1}^{*} \mid k_{t-2}^{*}, k_{t-3}^{*}, \mathbf{s}_{t}}$ without loss of generality as

\footnotetext{
${ }^{6}$ As long as $E\left[m_{t} \mid k_{t}, k_{t-1}, m_{t-1}, l_{t-1}\right]$ is not a function of $\left(m_{t-1}, k_{t-1}\right)$ only we do not need an additional excluded instrument for $m_{t}$ beyond $m_{t-1}$. Adding further lagged variables like $m_{t-2}$ as an excluded instrument for $m_{t}$ can improve efficiency.
} 


$$
\begin{aligned}
& f_{r_{t}, k_{t}^{*}, k_{t-1}^{*} \mid k_{t-2}^{*}, k_{t-3}^{*}, \mathbf{s}_{t}} \\
= & \int f_{r_{t}, k_{t}, k_{t-1}, k_{t}^{*}, k_{t-1}^{*} \mid k_{t-2}^{*}, k_{t-3}^{*}, \mathbf{s}_{t}} d k_{t} d k_{t-1} \\
= & \int f_{r_{t} \mid k_{t}, k_{t-1}, k_{t}^{*}, k_{t-1}^{*}, k_{t-2}^{*}, k_{t-3}^{*}, \mathbf{s}_{t}} f_{k_{t}, k_{t-1}, k_{t}^{*}, k_{t-1}^{*} \mid k_{t-2}^{*}, k_{t-3}^{*}, \mathbf{s}_{t}} d k_{t} d k_{t-1} \\
= & \int f_{r_{t} \mid k_{t}, k_{t-1}, k_{t}^{*}, k_{t-1}^{*}, k_{t-2}^{*}, k_{t-3}^{*}, \mathbf{s}_{t}} f_{k_{t}^{*}, k_{t-1}^{*} \mid k_{t}, k_{t-1}, k_{t-2}^{*}, k_{t-3}^{*}, \mathbf{s}_{t}} f_{k_{t}, k_{t-1} \mid k_{t-2}^{*}, k_{t-3}^{*}, \mathbf{s}_{t}} d k_{t} d k_{t-1} .
\end{aligned}
$$

where the first equality follows from the properties of densities and the second and third equalities follow from Bayes Rule.

Our key identification assumption is that current and lagged mismeasured capital inputs do not provide additional information on output and other inputs beyond what the true capital inputs do. This implies that the first term under the integral in the last line of equation (3) can be written as

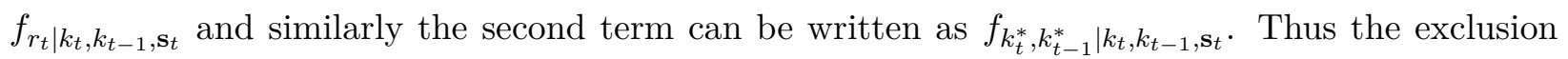
restriction allows us to re-express the last line of equation (3) as

$$
f_{r_{t}, k_{t}^{*}, k_{t-1}^{*} \mid k_{t-2}^{*}, k_{t-3}^{*}, \mathbf{s}_{t}}=\int f_{r_{t} \mid k_{t}, k_{t-1}, \mathbf{s}_{t}} f_{k_{t}^{*}, k_{t-1}^{*} \mid k_{t}, k_{t-1}, \mathbf{s}_{t}} f_{k_{t}, k_{t-1} \mid k_{t-2}^{*}, k_{t-3}^{*}, \mathbf{s}_{t}} d k_{t} d k_{t-1}
$$

Under weak regularity conditions and a standard rank condition for nonparametric instrumental variables we show in Section 4 that this equation has unique solutions for $f_{r_{t} \mid k_{t}, k_{t-1}, \mathbf{s}_{t}}, f_{k_{t}^{*}, k_{t-1}^{*} \mid k_{t}, k_{t-1}, \mathbf{s}_{t}}$,

and $f_{k_{t}, k_{t-1} \mid k_{t-2}^{*}, k_{t-3}^{*}, \mathbf{s}_{t}}$ following similar arguments to Hu and Schennach (2008). Given uniqueness a ML estimator can be used in a first stage to recover the conditional densities and a second stage uses these estimated conditional densities to construct the sample analog of the objective function (2), which is then used to recover estimates of $\theta_{0}$. The estimator extends directly to cases with measurement error in both capital and other inputs.

\section{A Generalized Approach in the Wooldridge (2005) Setting}

We first develop the method for the case when only capital is measured with error. We then generalize the setup to allow for measurement error in other inputs and capital.

\subsection{Measurement Error in Capital Only}

Let $\beta=\left(\beta_{l}, \beta_{k}, \beta_{m}, \beta_{\omega}\right), \theta=(\beta, q)$ and $\left(\beta_{0}, \theta_{0}\right)$ denote the true values. Following Wooldridge we define the residual functions as

$$
\begin{aligned}
\epsilon_{t}(\beta) \equiv \rho_{1}\left(\mathbf{y}_{t} ; \beta\right) & =y_{t}-\beta_{l} l_{t}-\beta_{k} k_{t}-\beta_{m} m_{t}-\mathbf{c}\left(m_{t}, k_{t}\right)^{\prime} \beta_{\omega} \\
\xi_{t}+\epsilon_{t}(\theta) \equiv \rho_{2}\left(\mathbf{y}_{t, t-1} ; \theta\right) & =y_{t}-\beta_{l} l_{t}-\beta_{k} k_{t}-\beta_{m} m_{t}-q\left(\mathbf{c}\left(m_{t-1}, k_{t-1}\right)^{\prime} \beta_{\omega}\right)
\end{aligned}
$$


where $\mathbf{y}_{t} \equiv\left(y_{t}, l_{t}, k_{t}, m_{t}\right)$ and $\mathbf{y}_{t, t-1} \equiv\left(\mathbf{y}_{t}, k_{t-1}, m_{t-1}\right)$. The first residual function corresponds to the i.i.d. shock and the second function corresponds to the composite error.

Let $\tilde{\mathbf{x}}_{t}=\left(k_{t}, k_{t-1}, m_{t-1}, l_{t-1}\right)$ and let $\mathbf{x}_{t}=\left(\tilde{\mathbf{x}}_{t}, l_{t}, m_{t}\right) \cdot{ }^{7}$ Then the conditional moment restrictions take the form

$$
\begin{aligned}
g_{1}\left(\mathbf{x}_{t} ; \beta\right) & \equiv E\left[\rho_{1}\left(\mathbf{y}_{t} ; \beta\right) \mid \mathbf{x}_{t}\right] \text { and } g_{1}\left(\mathbf{x}_{t} ; \beta_{0}\right)=0 \\
g_{2}\left(\tilde{\mathbf{x}}_{t} ; \theta\right) & \equiv E\left[\rho_{2}\left(\mathbf{y}_{t, t-1} ; \theta\right) \mid \tilde{\mathbf{x}}_{t}\right] \text { and } g_{2}\left(\tilde{\mathbf{x}}_{t} ; \theta_{0}\right)=0
\end{aligned}
$$

As noted by Wooldridge the second moment condition is sufficient for identification production function parameters.

Letting

$$
g\left(\mathbf{x}_{t} ; \theta\right)=\left(\begin{array}{c}
g_{1}\left(\mathbf{x}_{t} ; \beta\right) \\
g_{2}\left(\tilde{\mathbf{x}}_{t} ; \theta\right)
\end{array}\right)
$$

and defining $A=A\left(\mathbf{x}_{t}\right)$ to be some positive-definite weight matrix, the population objective function is given as

$$
\begin{aligned}
Q(\theta) & =E\left[g\left(\mathbf{x}_{t} ; \theta\right)^{\prime} A g\left(\mathbf{x}_{t} ; \theta\right)\right] \\
& =\int g\left(\mathbf{x}_{t} ; \theta\right)^{\prime} A g\left(\mathbf{x}_{t} ; \theta\right) f_{\mathbf{x}_{t}} d \mathbf{x}_{t} \\
& =\int g\left(\mathbf{x}_{t} ; \theta\right)^{\prime} A g\left(\mathbf{x}_{t} ; \theta\right) f_{k_{t}, k_{t-1} \mid m_{t}, l_{t}, m_{t-1}, l_{t-1}} f_{m_{t}, l_{t}, m_{t-1}, l_{t-1}} d \mathbf{x}_{t}
\end{aligned}
$$

Let $\mathcal{H}_{n}=\left\{q: q=p^{\kappa_{2 n}}\left(\mathbf{c}\left(m_{t-1}, k_{t-1}\right)^{\prime} \beta_{\omega}\right)^{\prime} \pi_{q}\right\}$ denote a sieve space to approximate the space of functions $q$ where $p^{\kappa_{2 n}}(\cdot)$ denotes approximating basis functions with length equal to $\kappa_{2 n}$ (e.g. polynomial approximations or spline approximations). In the first stage we recover estimates of $f_{y_{t} \mid k_{t}, k_{t-1}, m_{t}, l_{t}, m_{t-1}, l_{t-1}}, f_{y_{t} \mid k_{t}, k_{t-1}, m_{t-1}, l_{t-1}}, f_{l_{t} \mid k_{t}, k_{t-1}, m_{t-1}, l_{t-1}}$, and $f_{m_{t} \mid k_{t}, k_{t-1}, m_{t-1}, l_{t-1}}$. We also estimate $f_{k_{t}, k_{t-1} \mid m_{t}, l_{t}, m_{t-1}, l_{t-1}}$ in the first stage, which we need to integrate out the unobserved variables $\left(k_{t}, k_{t-1}\right)$ in sample objective function analog to (4). For any parameter value $\theta$ we use the first four densities to evaluate the conditional moment functions $\hat{g}\left(\mathbf{x}_{t} ; \theta\right)$ as

$$
\begin{aligned}
\hat{g}_{1}\left(\mathbf{x}_{t} ; \beta\right)= & \int y_{t} \hat{f}_{y_{t} \mid k_{t}, k_{t-1}, m_{t}, l_{t}, m_{t-1}, l_{t-1}} d y_{t}-\beta_{l} l_{t}-\beta_{k} k_{t}-\beta_{m} m_{t}-\mathbf{c}\left(m_{t}, k_{t}\right)^{\prime} \beta_{\omega} \\
\hat{g}_{2}\left(\tilde{\mathbf{x}}_{t} ; \theta\right)= & \int y_{t} \hat{f}_{y_{t} \mid k_{t}, k_{t-1}, m_{t-1}, l_{t-1}} d y_{t}-\beta_{l} \int l_{t} \hat{f}_{l_{t} \mid k_{t}, k_{t-1}, m_{t-1}, l_{t-1}} d l_{t}-\beta_{k} k_{t} \\
& -\beta_{m} \int m_{t} \hat{f}_{m_{t} \mid k_{t}, k_{t-1}, m_{t-1}, l_{t-1}} d m_{t}-q\left(\mathbf{c}\left(m_{t-1}, k_{t-1}\right)^{\prime} \beta_{\omega}\right) .
\end{aligned}
$$

\footnotetext{
${ }^{7}$ Under the assumptions maintained in Wooldridge's setup higher-order lagged values of inputs are also available as instruments.
} 
The sieve MD estimator is given as

$$
\hat{\theta}_{n}=\operatorname{argmin}_{\theta=(\beta, q), q \in \mathcal{H}_{n}} \widehat{Q}_{n}(\theta)
$$

where the sample objective function is

$$
\widehat{Q}_{n}(\theta)=\frac{1}{n} \sum_{i=1}^{n} \int \hat{g}\left(\mathbf{x}_{t i} ; \theta\right)^{\prime} \hat{A} \hat{g}\left(\mathbf{x}_{t i} ; \theta\right) \hat{f}_{k_{t}, k_{t-1} \mid m_{t, i}, l_{t, i}, m_{t-1, i}, l_{t-1, i}} d k_{t} d k_{t-1}
$$

with $\hat{A}$ denoting a consistent estimator of $A$ (e.g. Ai and Chen 2003).

\subsection{Measurement Error in Capital and Intermediate Inputs}

Our method can be extended to allow both capital and intermediate inputs to be measured with errors. Consider the production function

$$
y_{t}=\beta_{l} l_{t}+\beta_{k} k_{t}+\beta_{m} m_{t}+\omega_{t}+\epsilon_{t}
$$

where $\left(k_{t}, m_{t}\right)$ are not directly observable, but instead mismeasured inputs $\left(k_{t}^{*}, m_{t}^{*}\right)$ are observed. The population objective function is rewritten as

$$
Q(\theta)=\int g\left(\mathbf{x}_{t} ; \theta\right)^{\prime} A g\left(\mathbf{x}_{t} ; \theta\right) f_{k_{t}, k_{t-1}, m_{t}, m_{t-1} \mid l_{t}, l_{t-1}} f_{l_{t}, l_{t-1}} d \mathbf{x}_{t}
$$

where $f_{l_{t}, l_{t-1}}$ is observable from the data. The sieve MD estimator is defined as

$$
\hat{\theta}_{n}=\operatorname{argmin}_{\theta=(\beta, q), q \in \mathcal{H}_{n}} \widehat{Q}_{n}(\theta)
$$

where the sample objective function is given by

$$
\widehat{Q}_{n}(\theta)=\frac{1}{n} \sum_{i=1}^{n} \int \hat{g}\left(\mathbf{x}_{t i} ; \theta\right)^{\prime} \hat{A} \hat{g}\left(\mathbf{x}_{t i} ; \theta\right) \hat{f}_{k_{t}, k_{t-1}, m_{t}, m_{t-1} \mid l_{t, i}, l_{t-1, i}} d k_{t} d k_{t-1} d m_{t} d m_{t-1}
$$

By similar arguments to the results in Section 4 below we can identify the conditional density functions associated with latent true inputs, $\left(k_{t}, k_{t-1}, m_{t}, m_{t-1}\right)$ to construct the moment functions, given mismeasured inputs $\left(k_{t}^{*}, k_{t-1}^{*}, m_{t}^{*}, m_{t-1}^{*}\right)$. The identification problem in this case is only about adding more instruments because of the unobserved true intermediate inputs. The set of additional instruments required other than $\left(k_{t-2}^{*}, k_{t-3}^{*}\right)$ would be lags of mismeasured intermediate input $\left(m_{t-2}^{*}, m_{t-3}^{*}\right)$ or lags of other (possibly mismeasured) intermediate inputs.

To construct the moment functions we need to identify $f_{y_{t} \mid k_{t}, k_{t-1}, m_{t}, l_{t}, m_{t-1}, l_{t-1}}$ in $g_{1}\left(\mathbf{x}_{t} ; \beta\right)$, $f_{y_{t} \mid k_{t}, k_{t-1}, m_{t-1}, l_{t-1}}, f_{l_{t} \mid k_{t}, k_{t-1}, m_{t-1}, l_{t-1}}$, and $f_{m_{t} \mid k_{t}, k_{t-1}, m_{t-1}, l_{t-1}}$ in $g_{2}\left(\tilde{\mathbf{x}}_{t} ; \theta\right)$. With the true intermediate input $m_{t}$ being not observed, the only added complication is to identify the density $f_{m_{t} \mid k_{t}, k_{t-1}, m_{t-1}, l_{t-1}}$ because both $m_{t}$ and $m_{t-1}$ are not observed, which means both the regressor and 
the regressand are unobserved in a regression context. But given identification of $f_{k_{t}, k_{t-1}, m_{t}, m_{t-1} \mid l_{t-1}}$, by Bayes Rule, it is trivial to obtain

$$
f_{m_{t} \mid k_{t}, k_{t-1}, m_{t-1}, l_{t-1}}=\frac{f_{k_{t}, k_{t-1}, m_{t}, m_{t-1} \mid l_{t-1}}}{f_{k_{t}, k_{t-1}, m_{t-1} \mid l_{t-1}}}=\frac{f_{k_{t}, k_{t-1}, m_{t}, m_{t-1} \mid l_{t-1}}}{\int f_{k_{t}, k_{t-1}, m_{t}, m_{t-1} \mid l_{t-1}} d m_{t}} .
$$

The conditional density functions, $f_{k_{t}, k_{t-1}, m_{t}, m_{t-1} \mid l_{t}, l_{t-1}}$ in $Q(\theta)$ and $f_{k_{t}, k_{t-1}, m_{t}, m_{t-1} \mid l_{t-1}}$ in (6) associated with latent true inputs, $\left(k_{t}, k_{t-1}, m_{t}, m_{t-1}\right)$, can be also identified by similar arguments to the results in Section 4. Given identification results we then estimate $\hat{g}\left(\mathbf{x}_{t} ; \theta\right)$ and $\hat{f}_{k_{t}, k_{t-1}, m_{t}, m_{t-1} \mid l_{t}, l_{t-1}}$ in $\widehat{Q}_{n}(\theta)$ where $\left(k_{t}, k_{t-1}, m_{t}, m_{t-1}\right)$ are unobserved, but instead $\left(k_{t}^{*}, k_{t-1}^{*}, m_{t}^{*}, m_{t-1}^{*}\right)$ are observed using (e.g.) lagged values $\left(k_{t-2}^{*}, k_{t-3}^{*}, m_{t-2}^{*}, m_{t-3}^{*}\right)$ as instruments.

\section{Identification and Estimation}

In this section we develop identification and estimation of conditional density functions that appear in the moment functions.

\subsection{Identification of Density Functions}

In order to implement the sieve MD estimation (5) we need to obtain consistent estimators of $f_{y_{t} \mid k_{t}, k_{t-1}, m_{t}, l_{t}, m_{t-1}, l_{t-1}}, f_{y_{t} \mid k_{t}, k_{t-1}, m_{t-1}, l_{t-1}}, f_{l_{t} \mid k_{t}, k_{t-1}, m_{t-1}, l_{t-1}}, f_{m_{t} \mid k_{t}, k_{t-1}, m_{t-1}, l_{t-1}}$, and $f_{k_{t}, k_{t-1} \mid m_{t}, l_{t}, m_{t-1}, l_{t-1}}$. Let either (i) $r_{t}=y_{t}$ and $\mathbf{s}_{t}=\left(m_{t}, l_{t}, m_{t-1}, l_{t-1}\right)$ or (ii) $r_{t} \in\left\{l_{t}, m_{t}\right\}$ and $\mathbf{s}_{t}=\left(m_{t-1}, l_{t-1}\right)$, depending on problems of interest.

\subsubsection{Identification of $f_{r_{t} \mid k_{t}, k_{t-1}, \mathbf{s}_{t}}$}

We make the following assumptions for identification and discussions follow. ${ }^{8}$

\section{Assumption 4.1.}

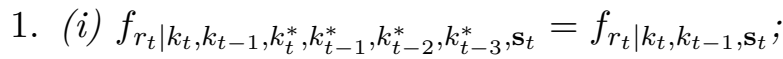

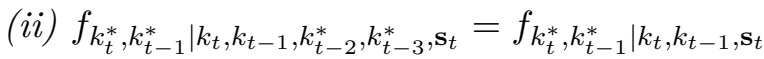

2. The operators $L_{k_{t}^{*}, k_{t-1}^{*} \mid k_{t-2}^{*}, k_{t-3}^{*}, \mathbf{s}_{t}}$ and $L_{k_{t}^{*}, k_{t-1}^{*} \mid k_{t}, k_{t-1}, \mathbf{s}_{t}}$ (defined in the Appendix A) are oneto-one

3. For any $\mathbf{s}_{t} \in \operatorname{Supp}\left(\mathbf{s}_{t}\right)$ and any $\left(\tilde{k}_{t}, \tilde{k}_{t-1}\right) \neq\left(\bar{k}_{t}, \bar{k}_{t-1}\right) \in \operatorname{Supp}\left(k_{t}, k_{t-1}\right)$, the set

$$
\left\{r_{t}: f_{r_{t} \mid k_{t}, k_{t-1}, \mathbf{s}_{t}}\left(r_{t} \mid \tilde{k}_{t}, \tilde{k}_{t-1}, \mathbf{s}_{t}\right) \neq f_{r_{t} \mid k_{t}, k_{t-1}, \mathbf{s}_{t}}\left(r_{t} \mid \bar{k}_{t}, \bar{k}_{t-1}, \mathbf{s}_{t}\right)\right\}
$$

\footnotetext{
${ }^{8}$ We focus on a decomposition-based approach as in Hu and Schennach (2008). It is worth noting that our identification strategy to recover production function parameters is not only restricted to their approach. Other nonlinear methods to recover true densities from observed data (e.g., repeated measurement or auxiliary data approach) can be also used for this purpose.
} 
has positive probability

4. For any given $\mathbf{s}_{t} \in S u p p\left(\mathbf{s}_{t}\right)$, there exists a known functional $M$ such that for all $\left(k_{t}, k_{t-1}\right) \in$ $\operatorname{Supp}\left(k_{t}, k_{t-1}\right)$

$$
M\left[f_{k_{t}^{*}, k_{t-1}^{*} \mid k_{t}, k_{t-1}, \mathbf{s}_{t}}\left(\cdot \mid k_{t}, k_{t-1}, \mathbf{s}_{t}\right)\right]=\left(k_{t}, k_{t-1}\right) .
$$

Assumption 4.1 .1 (i) is an exclusion restriction that $\left(k_{t}^{*}, k_{t-1}^{*}, k_{t-2}^{*}, k_{t-3}^{*}\right)$ do not provide additional information on $r_{t}$ beyond what is known conditional on $\left(k_{t}, k_{t-1}, \mathbf{s}_{t}\right)$. Assumption 4.1.1 (ii) is a similar exclusion restriction that $\left(k_{t-2}^{*}, k_{t-3}^{*}\right)$ do not provide additional information on $\left(k_{t}^{*}, k_{t-1}^{*}\right)$ beyond what is known conditional on $\left(k_{t}, k_{t-1}, \mathbf{s}_{t}\right)$. Assumption 4.1.2 is a nonparametric rank condition of the instruments and is important for the invertibility of the integral operators we employ for our identification proof. This identification condition appears in several different forms in the literature. For example it has been phrased as singular value decompositions with nonzero singular values (Darolles, Fan, Florens, and Renault 2011), nonsingularity (Hall and Horowitz 2005, Horowitz 2006), or completeness condition (or bounded completeness) (Newey and Powell 2003, Blundell, Chen, and Kristensen 2007, Andrews 2011).

Assumption 4.1.3 states the conditional density functions of the output and other inputs are distinguishable at two distinct values of capital. Assumption 4.1.3 ensures the uniqueness of the decomposition of an integral operator associated with $f_{\cdot \mid k_{t}, k_{t-1}, \mathbf{s}_{t}}$. This condition is violated only if the distribution of $r_{t}$ conditional on $\left(k_{t}, k_{t-1}, \mathbf{s}_{t}\right)$ is identical at different values of $\left(k_{t}, k_{t-1}\right)$. For output this condition holds as long as $\beta_{k}>0$.

Assumption 4.1.4 places restrictions on some measure of the location of a density, denoted by $M[\cdot]$ such as the mean, mode, and quantiles of the distribution. For example the classical measurement error imposes $E\left[k_{t}^{*} \mid k_{t}\right]=k_{t}$ while the nonclassical measurement error extends to the case e.g. median $\left(k_{t}^{*} \mid k_{t}\right)=k_{t}$, which ensures the uniqueness of the decomposition of an integral operator associated with $f_{\cdot \mid k_{t}, k_{t-1}, \mathbf{s}_{t}}$.

Assumption 4.1 is sufficient for identification of the density functions $f_{r_{t} \mid k_{t}, k_{t-1}, \mathbf{s}_{t}}, f_{k_{t}^{*}, k_{t-1}^{*} \mid k_{t}, k_{t-1}, \mathbf{s}_{t}}$, and $f_{k_{t}, k_{t-1} \mid k_{t-2}^{*}, k_{t-3}^{*}, \mathbf{s}_{t}}$.

Theorem 4.2. Suppose Assumption 4.1 holds. Then the observations of $\left(r_{t}, k_{t}^{*}, k_{t-1}^{*}, k_{t-2}^{*}, k_{t-3}^{*}, \mathbf{s}_{t}\right)$ uniquely identify unobserved densities

$$
f_{r_{t} \mid k_{t}, k_{t-1}, \mathbf{s}_{t}}, f_{k_{t}^{*}, k_{t-1}^{*} \mid k_{t}, k_{t-1}, \mathbf{s}_{t}}, \text { and } f_{k_{t}, k_{t-1} \mid k_{t-2}^{*}, k_{t-3}^{*}, \mathbf{s}_{t}}
$$

\subsubsection{Identification of $f_{k_{t}, k_{t-1} \mid m_{t}, l_{t}, m_{t-1}, l_{t-1}}$ and $f_{y_{t} \mid k_{t}, k_{t-1}, m_{t-1}, l_{t-1}}$}

Next we show that other conditional density functions that appear in the moment functions and the objective function are obtained from both observables and identified objects from the previous section. Using the results in Theorem 4.2 we also identify $f_{k_{t}, k_{t-1} \mid m_{t}, l_{t}, m_{t-1}, l_{t-1}}$ as 


$$
f_{k_{t}, k_{t-1} \mid m_{t}, l_{t}, m_{t-1}, l_{t-1}}=\int f_{k_{t}, k_{t-1} \mid k_{t-2}^{*}, k_{t-3}^{*}, m_{t}, l_{t}, m_{t-1}, l_{t-1}} \frac{f_{k_{t-2}^{*}, k_{t-3}^{*}, m_{t}, l_{t}, m_{t-1}, l_{t-1}}}{f_{m_{t}, l_{t}, m_{t-1}, l_{t-1}}} d k_{t-2}^{*} d k_{t-3}^{*}
$$

because $f_{k_{t}, k_{t-1} \mid k_{t-2}^{*}, k_{t-3}^{*}, m_{t}, l_{t}, m_{t-1}, l_{t-1}}$ is identified from Theorem 4.2 and $f_{k_{t-2}^{*}, k_{t-3}^{*}, m_{t}, l_{t}, m_{t-1}, l_{t-1}}$ and $f_{m_{t}, l_{t}, m_{t-1}, l_{t-1}}$ are observed from the data. The following Corollary summarizes the result.

Corollary 4.3. Suppose Assumption 4.1 holds. Then we identify $f_{k_{t}, k_{t-1} \mid m_{t}, l_{t}, m_{t-1}, l_{t-1}}$ from the observations of $\left(y_{t}, k_{t}^{*}, k_{t-1}^{*}, k_{t-2}^{*}, k_{t-3}^{*}, m_{t}, l_{t}, m_{t-1}, l_{t-1}\right)$.

We next identify $f_{y_{t} \mid k_{t}, k_{t-1}, m_{t-1}, l_{t-1}}$. Note that

$$
f_{y_{t} \mid k_{t}, k_{t-1}, m_{t-1}, l_{t-1}}=\int f_{y_{t} \mid k_{t}, k_{t-1}, m_{t}, l_{t}, m_{t-1}, l_{t-1}} f_{m_{t}, l_{t} \mid k_{t}, k_{t-1}, m_{t-1}, l_{t-1}} d m_{t} d l_{t}
$$

and

$$
\begin{aligned}
f_{m_{t}, l_{t} \mid k_{t}, k_{t-1}, m_{t-1}, l_{t-1}} & =f_{m_{t}, l_{t}, k_{t}, k_{t-1} \mid m_{t-1}, l_{t-1}} \frac{f_{m_{t-1}, l_{t-1}}}{f_{k_{t}, k_{t-1}, m_{t-1}, l_{t-1}}} \\
& =f_{k_{t}, k_{t-1} \mid m_{t}, l_{t}, m_{t-1}, l_{t-1}} \frac{f_{m_{t}, l_{t}, m_{t-1}, l_{t-1}}}{f_{m_{t-1}, l_{t-1}}} \frac{f_{m_{t-1}, l_{t-1}}}{f_{k_{t}, k_{t-1}, m_{t-1}, l_{t-1}}} \\
& =f_{k_{t}, k_{t-1} \mid m_{t}, l_{t}, m_{t-1}, l_{t-1}} \frac{f_{m_{t}, l_{t}, m_{t-1}, l_{t-1}}}{f_{k_{t}, k_{t-1}, m_{t-1}, l_{t-1}}} .
\end{aligned}
$$

Then

$$
f_{y_{t} \mid k_{t}, k_{t-1}, m_{t-1}, l_{t-1}}=\int f_{y_{t} \mid k_{t}, k_{t-1}, m_{t}, l_{t}, m_{t-1}, l_{t-1}} f_{k_{t}, k_{t-1} \mid m_{t}, l_{t}, m_{t-1}, l_{t-1}} \frac{f_{m_{t}, l_{t}, m_{t-1}, l_{t-1}}}{f_{k_{t}, k_{t-1}, m_{t-1}, l_{t-1}}} d m_{t} d l_{t}
$$

Also note that

$$
\begin{aligned}
f_{k_{t}, k_{t-1}, m_{t-1}, l_{t-1}} & =\int f_{k_{t}, k_{t-1}, k_{t-2}^{*}, k_{t-3}^{*}, m_{t}, l_{t}, m_{t-1}, l_{t-1}} d k_{t-2}^{*} d k_{t-3}^{*} d m_{t} d l_{t} \\
& =\int f_{k_{t}, k_{t-1} \mid k_{t-2}^{*}, k_{t-3}^{*}, m_{t}, l_{t}, m_{t-1}, l_{t-1}} f_{k_{t-2}^{*}, k_{t-3}^{*}, m_{t}, l_{t}, m_{t-1}, l_{t-1}} d k_{t-2}^{*} d k_{t-3}^{*} d m_{t} d l_{t}
\end{aligned}
$$

Because (i) $f_{y_{t} \mid k_{t}, k_{t-1}, m_{t}, l_{t}, m_{t-1}, l_{t-1}}$ and $f_{k_{t}, k_{t-1} \mid k_{t-2}^{*}, k_{t-3}^{*}, m_{t}, l_{t}, m_{t-1}, l_{t-1}}$ are identified from Theorem 4.2 and (ii) $f_{k_{t}, k_{t-1} \mid m_{t}, l_{t}, m_{t-1}, l_{t-1}}$ is identified from Corollary 4.3 and because $f_{m_{t}, l_{t}, m_{t-1}, l_{t-1}}$ and $f_{k_{t-2}^{*}, k_{t-3}^{*}, m_{t}, l_{t}, m_{t-1}, l_{t-1}}$ are observed from the data (by a nonparametric density estimation), we conclude $f_{y_{t} \mid k_{t}, k_{t-1}, m_{t-1}, l_{t-1}}$ is also identified from (7) and (8). The following Corollary summarizes the result.

Corollary 4.4. Suppose Assumption 4.1 holds. Then we identify $f_{y_{t} \mid k_{t}, k_{t-1}, m_{t-1}, l_{t-1}}$ from the observations of $\left(y_{t}, k_{t}^{*}, k_{t-1}^{*}, k_{t-2}^{*}, k_{t-3}^{*}, m_{t}, l_{t}, m_{t-1}, l_{t-1}\right)$. 


\subsection{Estimation of Conditional Densities}

In the sieve MD estimation (5), we first estimate conditional densities that enter $g_{1}\left(\mathbf{x}_{t} ; \beta\right)$ and $g_{2}\left(\tilde{\mathbf{x}}_{t} ; \theta\right)$ for given $\theta \equiv(\beta, q)$ with $\beta \equiv\left(\beta_{l}, \beta_{k}, \beta_{m}, \beta_{\omega}\right)$ and the conditional density that is required to integrate out unobserved variables, $\left(k_{t}, k_{t-1}\right)$, in $Q(\theta)$. Then we estimate $\theta$ in the second stage. So in the first stage, we need to estimate conditional densities associated with unobserved variables, $\left(k_{t}, k_{t-1}\right)$, such as $f_{k_{t}, k_{t-1} \mid m_{t}, l_{t}, m_{t-1}, l_{t-1}}, f_{y_{t} \mid k_{t}, k_{t-1}, m_{t}, l_{t}, m_{t-1}, l_{t-1}}, f_{y_{t} \mid k_{t}, k_{t-1}, m_{t-1}, l_{t-1}}, f_{l_{t} \mid k_{t}, k_{t-1}, m_{t-1}, l_{t-1}}$, and $f_{m_{t} \mid k_{t}, k_{t-1}, m_{t-1}, l_{t-1}}$. The first conditional density is required to integrate out unobserved variables and the second one is needed to estimate $g_{1}\left(\mathbf{x}_{t} ; \beta\right)$. Last three conditional densities are needed to estimate $g_{2}\left(\tilde{\mathbf{x}}_{t} ; \theta\right)$. Recall that $\tilde{\mathbf{x}}_{t}=\left(k_{t}, k_{t-1}, m_{t-1}, l_{t-1}\right)$ and $\mathbf{x}_{t}=\left(\tilde{\mathbf{x}}_{t}, l_{t}, m_{t}\right)$. Also recall that when $r_{t}=y_{t}$, we take $\mathbf{s}_{t}=\left(m_{t}, l_{t}, m_{t-1}, l_{t-1}\right)$ and when $r_{t} \in\left\{l_{t}, m_{t}\right\}$, we take $\mathbf{s}_{t}=\left(m_{t-1}, l_{t-1}\right)$. Similarly, we let $\mathbf{z}_{t}=\left(k_{t}, k_{t-1}, \mathbf{s}_{t}\right)$ and $\mathbf{z}_{t}^{*}=\left(k_{t-2}^{*}, k_{t-3}^{*}, \mathbf{s}_{t}\right)$. We now discuss how to estimate those conditional densities.

\subsubsection{Estimation of $f_{r_{t} \mid k_{t}, k_{t-1}, \mathbf{s}_{t}}$}

Let

$$
\begin{aligned}
& f_{r_{t}, k_{t}^{*}, k_{t-1}^{*} \mid \mathbf{z}_{t}}\left(r_{t}, k_{t}, k_{t-1} \mid \mathbf{z}_{t} ; \alpha_{0}\right) \\
= & \int f_{r_{t} \mid \mathbf{z}_{t}}\left(r_{t} \mid \mathbf{z}_{t} ; \psi_{0}\right) f_{k_{t}^{*}, k_{t-1}^{*} \mid \mathbf{z}_{t}}\left(k_{t}^{*}, k_{t-1}^{*} \mid \mathbf{z}_{t}\right) f_{k_{t}, k_{t-1} \mid \mathbf{z}_{t}^{*}}\left(k_{t}, k_{t-1} \mid \mathbf{z}_{t}^{*}\right) d k_{t} d k_{t-1},
\end{aligned}
$$

where $\alpha_{0} \equiv\left(\psi_{0}, f_{1}, f_{2}\right) \in \mathcal{A} \equiv \Psi \times \mathcal{F}_{1} \times \mathcal{F}_{2}, \psi_{0} \equiv\left(\phi_{0}, \eta_{0}\right) \in \Psi \equiv \Phi \times \mathcal{M}, f_{1} \equiv f_{k_{t}^{*}, k_{t-1}^{*} \mid \mathbf{z}_{t}}\left(k_{t}^{*}, k_{t-1}^{*} \mid \mathbf{z}_{t}\right)$, and $f_{2} \equiv f_{k_{t}, k_{t-1} \mid \mathbf{z}_{t}^{*}}\left(k_{t}, k_{t-1} \mid \mathbf{z}_{t}^{*}\right)$. Here $\phi_{0}$ denotes a finite-dimensional parameter while $\eta_{0}$ denotes an infinite-dimensional parameter that determines the density $f_{r_{t} \mid \mathbf{z}_{t}}$. Therefore in this specification we use a semi-nonparametric density for $f_{r_{t} \mid \mathbf{z}_{t}}$ while we use nonparametric densities for $f_{k_{t}^{*}, k_{t-1}^{*} \mid \mathbf{z}_{t}}$

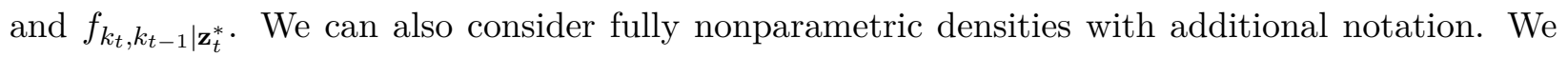
choose to use the current specification as (9) for flexibility.

From Theorem 4.2, the parameters can be recovered by solving the maximization problems:

$$
\begin{aligned}
\alpha_{0}= & \arg \max _{\alpha \equiv\left(\psi, f_{1}, f_{2}\right) \in \mathcal{A}} \\
& E\left[\ln \int f_{r_{t} \mid \mathbf{z}_{t}}\left(r_{t} \mid \mathbf{z}_{t} ; \psi_{0}\right) f_{1}\left(k_{t}^{*}, k_{t-1}^{*} \mid \mathbf{z}_{t}\right) f_{2}\left(k_{t}, k_{t-1} \mid \mathbf{z}_{t}^{*}\right) d k_{t} d k_{t-1}\right] .
\end{aligned}
$$

Note that $\Phi$ is finite-dimensional parameter space. But we need to impose some restrictions on the function spaces $\left(\mathcal{M}, \mathcal{F}_{1}, \mathcal{F}_{2}\right)$ for infinite-dimensional parameters. Let $\xi \in \mathcal{V} \subset \mathcal{R}^{d_{\xi}},\|\cdot\|_{E}$ denote the Euclidean norm, and

$$
\nabla^{\mathbf{a}} \mathrm{g}(\xi) \equiv \frac{\partial^{a_{1}+a_{2}+\cdots+a_{d_{\xi}}} g(\xi)}{\partial \xi_{1}^{a_{1}} \cdots \partial \xi_{d_{\xi}}^{a_{d_{\xi}}}}
$$

denote the $\sum_{i=1}^{d_{\xi}} a_{i}$-th derivative where $\mathbf{a}=\left(a_{1}, a_{2}, \ldots, a_{d_{\xi}}\right)^{\prime}$ is a vector of nonnegative integers. 
Let $\underline{\gamma}$ denote the largest integer satisfying $\underline{\gamma}<\gamma$. The Hölder space $\Lambda^{\gamma}(\mathcal{V})$ of order $\gamma>0$ is a space of functions $\mathrm{g}: \mathcal{V} \mapsto \mathcal{R}$ such that the first $\underline{\gamma}$ derivatives are bounded and the $\underline{\gamma}$-th derivative is Hölder continuous with the exponent $\gamma-\underline{\gamma} \in(0,1]$, i.e., for all $\xi, \xi^{\prime} \in \mathcal{V}$ and some constant $c$

$$
\max _{\sum_{i=1}^{d_{\xi}} a_{i}=\underline{\gamma}}\left|\nabla^{\mathbf{a}} \mathrm{g}(\xi)-\nabla^{\mathbf{a}} \mathrm{g}\left(\xi^{\prime}\right)\right| \leq c\left(\left\|\xi-\xi^{\prime}\right\|_{E}\right)^{\gamma-\underline{\gamma}}
$$

The space $\Lambda^{\gamma}(\mathcal{V})$ becomes a Banach space under the Hölder norm:

$$
\|\mathrm{g}\|_{\Lambda^{\gamma}}=\sup _{\xi}|\mathrm{g}(\xi)|+\max _{\sum_{i=1}^{d_{\xi}} a_{i}=\underline{\gamma} \xi \neq \xi^{\prime}} \sup _{\underline{\xi}} \frac{\left|\nabla^{\mathbf{a}} \mathrm{g}(\xi)-\nabla^{\mathbf{a}} \mathrm{g}\left(\xi^{\prime}\right)\right|}{\left(\left\|\xi-\xi^{\prime}\right\|_{E}\right)^{\gamma-\underline{\gamma}}}<\infty .
$$

A Hölder ball (of radius $c$ ) is defined as $\Lambda_{c}^{\gamma}(\mathcal{V}) \equiv\left\{\mathrm{g} \in \Lambda^{\gamma}(\mathcal{V}):\|\mathrm{g}\|_{\Lambda^{\gamma}} \leq c<\infty\right\}$. Let $\nu(\cdot$ ) be a positive continuous weight function on $\mathcal{V}$ where $\nu(\xi)=\left(1+\|\xi\|_{E}^{2}\right)^{-\varsigma / 2}, \varsigma>\gamma>0$. Denote $\Lambda_{c}^{\gamma, \nu}(\mathcal{V})$ as the weighted Hölder space with a weighted Hölder norm $\|\mathrm{g}\|_{\Lambda^{\gamma, \nu}} \equiv\|\tilde{\mathrm{g}}\|_{\Lambda^{\gamma}}$ for $\tilde{\mathrm{g}}(\xi) \equiv \mathrm{g}(\xi) \nu(\xi)$. Then a weighted Hölder ball is defined as $\Lambda_{c}^{\gamma, \nu}(\mathcal{V}) \equiv\left\{g \in \Lambda^{\gamma, \nu}(\mathcal{V}):\|g\|_{\Lambda^{\gamma, \nu}} \leq c<\infty\right\}$.

\section{Assumption 4.5.}

1. $\eta \in \Lambda_{c}^{\gamma_{1}, \nu}(\operatorname{Supp}(\cdot))$ where $\gamma_{1}>1$

2. $f_{1} \in \Lambda_{c}^{\gamma_{1}, \nu}\left(\operatorname{Supp}\left(k_{t}^{*}, k_{t-1}^{*}, \mathbf{z}_{t}\right)\right)$ where $\gamma_{1}>1$ and $\int f_{1}\left(k_{t}^{*}, k_{t-1}^{*} \mid \mathbf{z}_{t}\right) d k_{t}^{*} d k_{t-1}^{*}=1$ for all $\mathbf{z}_{t} \in$ $\operatorname{Supp}\left(\mathbf{z}_{t}\right)$

3. $f_{2} \in \Lambda_{c}^{\gamma_{1}, \nu}\left(\operatorname{Supp}\left(k_{t}, k_{t-1}, \mathbf{z}_{t}^{*}\right)\right)$ where $\gamma_{1}>1$ and $\int f_{2}\left(k_{t}, k_{t-1} \mid \mathbf{z}_{t}^{*}\right) d k_{t} d k_{t-1}=1$ for all $\mathbf{z}_{t}^{*} \in$ $\operatorname{Supp}\left(\mathbf{z}_{t}^{*}\right)$.

We define the spaces which satisfy relevant restrictions:

$$
\begin{aligned}
\mathcal{M} & =\{\eta(\cdot): \text { Assumption 4.5.1 holds }\} \\
\mathcal{F}_{1} & =\left\{f_{1}(\cdot, \cdot \mid \cdot): \text { Assumption 4.1.2, 4.1.4, and 4.5.2 hold }\right\}, \\
\mathcal{F}_{2} & =\left\{f_{2}(\cdot, \cdot \mid \cdot): \text { Assumption 4.1.2 and 4.5.3 hold }\right\} .
\end{aligned}
$$

Let $\left\{p_{j}, j=1,2, \ldots\right\}$ denote a sequence of known univariate basis functions (e.g., Fourier series, power series, spline, wavelets, etc.). For $i \in\{\eta, 1,2\}$, we define $p^{k_{n_{i}}}=\left(p_{1}, \ldots, p_{k_{n_{i}}}\right)$ as a tensorproduct linear sieve basis. For the consistent estimation of $\alpha_{0}$, we replace the parameter spaces with finite-dimensional compact sieve spaces:

$\mathcal{M}_{n}=\left\{\eta(\cdot)=p^{\kappa_{n \eta}}(\cdot)^{\prime} \pi_{\eta}\right.$ for all $\pi_{\eta}$ s.t. Assumption 4.5.1 holds $\}$,

$\mathcal{F}_{1 n}=\left\{f_{1}\left(k_{t}^{*}, k_{t-1}^{*} \mid \mathbf{z}_{t}\right)=p^{\kappa_{n_{1}}}\left(k_{t}^{*}, k_{t-1}^{*}, \mathbf{z}_{t}\right)^{\prime} \pi_{1}\right.$ for all $\pi_{1}$ s.t. Assumption 4.1.2, 4.1.4, and 4.5.2 hold $\}$, $\mathcal{F}_{2 n}=\left\{f_{2}\left(k_{t}, k_{t-1} \mid \mathbf{z}_{t}^{*}\right)=p^{\kappa_{n_{2}}}\left(k_{t}, k_{t-1}, \mathbf{z}_{t}^{*}\right)^{\prime} \pi_{2}\right.$ for all $\pi_{2}$ s.t. Assumption 4.1.2 and 4.5.3 hold $\}$. 
We then can replace the densities, $f_{1}$ and $f_{2}$ with functions in the sieve spaces:

$$
\begin{aligned}
f_{1}\left(k_{t}^{*}, k_{t-1}^{*} \mid \mathbf{z}_{t}\right) & =\sum_{j_{1}=0}^{j_{1 n}} \sum_{j_{2}=0}^{j_{2 n}} \sum_{j_{3}=0}^{j_{3 n}} \varphi_{j_{1} j_{2} j_{3}} p_{j_{1}}\left(k_{t}^{*}-k_{t-1}^{*}\right) p_{j_{2}}\left(k_{t-1}^{*}\right) p_{j_{3}}\left(\mathbf{z}_{t}\right), \\
f_{2}\left(k_{t}, k_{t-1} \mid \mathbf{z}_{t}^{*}\right) & =\sum_{j_{1}=0}^{j_{1 n}} \sum_{j_{2}=0}^{j_{2 n}} \sum_{j_{3}=0}^{j_{3 n}} \gamma_{j_{1} j_{2} j_{3}} p_{j_{1}}\left(k_{t}-k_{t-1}\right) p_{j_{2}}\left(k_{t-1}\right) p_{j_{3}}\left(\mathbf{z}_{t}^{*}\right) .
\end{aligned}
$$

Let the projection of the true parameter $\alpha_{0}$ onto the space $\mathcal{A}_{n}$ where $\mathcal{A}_{n}=\Psi_{n} \times \mathcal{F}_{1 n} \times \mathcal{F}_{2 n}$ with $\Psi_{n}=\Phi \times \mathcal{M}_{n}:$

$$
\begin{aligned}
\Pi_{n} \alpha & \equiv \alpha_{n} \\
& =\arg \max _{\alpha_{n} \equiv\left(\psi, f_{1}, f_{2}\right) \in \mathcal{A}_{n}} E\left[\ln \int f_{r_{t} \mid \mathbf{z}_{t}}\left(r_{t} \mid \mathbf{z}_{t} ; \psi\right) f_{1}\left(k_{t}^{*}, k_{t-1}^{*} \mid \mathbf{z}_{t}\right) f_{2}\left(k_{t}, k_{t-1} \mid \mathbf{z}_{t}^{*}\right) d k_{t} d k_{t-1}\right] .
\end{aligned}
$$

Then the proposed sieve ML estimators of $\alpha_{0}$ is defined as follows:

$$
\begin{aligned}
& \hat{\alpha}_{n} \equiv\left(\hat{\psi}_{n}, \hat{f}_{1 n}, \hat{f}_{2 n}\right) \\
= & \arg \max _{\left(\psi, f_{1}, f_{2}\right) \in \mathcal{A}_{n}} \frac{1}{n} \sum_{i=1}^{n} \ln \int f_{r_{t} \mid \mathbf{z}_{t}}\left(r_{t, i} \mid \mathbf{z}_{t, i} ; \psi\right) f_{1}\left(k_{t, i}^{*}, k_{t-1, i}^{*} \mid \mathbf{z}_{t, i}\right) f_{2}\left(k_{t}, k_{t-1} \mid \mathbf{z}_{t, i}^{*}\right) d k_{t} d k_{t-1}
\end{aligned}
$$

\subsubsection{Estimation of $f_{k_{t}, k_{t-1} \mid m_{t}, l_{t}, m_{t-1}, l_{t-1}}$ and $f_{y_{t} \mid k_{t}, k_{t-1}, m_{t-1}, l_{t-1}}$}

Based on Corollary 4.3, we propose the following estimator of $f_{k_{t}, k_{t-1} \mid m_{t}, l_{t}, m_{t-1}, l_{t-1}}$

$$
\hat{f}_{k_{t}, k_{t-1} \mid m_{t}, l_{t}, m_{t-1}, l_{t-1}}=\frac{\int \hat{f}_{k_{t}, k_{t-1} \mid k_{t-2}^{*}, k_{t-3}^{*}, m_{t}, l_{t}, m_{t-1}, l_{t-1}} \hat{f}_{k_{t-2}^{*}, k_{t-3}^{*}, m_{t}, l_{t}, m_{t-1}, l_{t-1}} d k_{t-2}^{*} d k_{t-3}^{*}}{\hat{f}_{m_{t}, l_{t}, m_{t-1}, l_{t-1}}}
$$

where $\hat{f}_{k_{t}, k_{t-1} \mid k_{t-2}^{*}, k_{t-3}^{*}, m_{t}, l_{t}, m_{t-1}, l_{t-1}}$ is $\hat{f}_{2}\left(k_{t}, k_{t-1} \mid \mathbf{z}_{t}^{*}\right)$ from the sieve ML estimation in the previous section. $\left(\hat{f}_{k_{t-2}^{*}}^{*}, k_{t-3}^{*}, m_{t}, l_{t}, m_{t-1}, l_{t-1}, \hat{f}_{m_{t}, l_{t}, m_{t-1}, l_{t-1}}\right)$ can be estimated by any consistent nonparametric density estimators of observables. Similarly, based on Corollary 4.4, we can estimate $f_{y_{t} \mid k_{t}, k_{t-1}, m_{t-1}, l_{t-1}}$ as

$$
\begin{aligned}
& \hat{f}_{y_{t} \mid k_{t}, k_{t-1}, m_{t-1}, l_{t-1}} \\
& =\frac{\int \hat{f}_{y_{t} \mid k_{t}, k_{t-1}, m_{t}, l_{t}, m_{t-1}, l_{t-1}} \hat{f}_{k_{t}, k_{t-1} \mid m_{t}, l_{t}, m_{t-1}, l_{t-1}} \hat{f}_{m_{t}, l_{t}, m_{t-1}, l_{t-1}} d m_{t} d l_{t}}{\int \hat{f}_{k_{t}, k_{t-1} \mid k_{t-2}^{*}, k_{t-3}^{*}, m_{t}, l_{t}, m_{t-1}, l_{t-1}} \hat{f}_{k_{t-2}^{*}, k_{t-3}^{*}, m_{t}, l_{t}, m_{t-1}, l_{t-1}} d k_{t-2}^{*} d k_{t-3}^{*} d m_{t} d l_{t}}
\end{aligned}
$$

where $\hat{f}_{y_{t} \mid k_{t}, k_{t-1}, m_{t}, l_{t}, m_{t-1}, l_{t-1}}$ is $\hat{f}_{r_{t} \mid \mathbf{z}_{t}}\left(r_{t} \mid \mathbf{z}_{t} ; \psi\right)$ for $r_{t}=y_{t}$ from the sieve ML estimation. To summarize, $\left(\hat{f}_{k_{t}, k_{t-1} \mid m_{t}, l_{t}, m_{t-1}, l_{t-1}}, \hat{f}_{y_{t} \mid k_{t}, k_{t-1}, m_{t-1}, l_{t-1}}\right)$ can be estimated by the sieve ML estimation obtained from (10) and consistent estimators of $\left(\hat{f}_{k_{t-2}^{*}, k_{t-3}^{*}, m_{t}, l_{t}, m_{t-1}, l_{t-1}}, \hat{f}_{m_{t}, l_{t}, m_{t-1}, l_{t-1}}\right)$ from nonparametric density estimation with observable data. 


\section{Consistency and Convergence rate}

Our estimation approach is a two-step method such that in the first stage we estimate conditional density functions associated with moment functions and in the second stage we estimate production function parameters. Our first stage to estimate conditional density functions follows $\mathrm{Hu}$ and Schennach (2008). Recall that $\alpha$ denote collections of different kinds of conditional density functions and parameters that determine the conditional density functions, which we need to estimate in the first stage. Also recall that $\beta=\left(\beta_{l}, \beta_{k}, \beta_{m}, \beta_{\omega}\right), \theta=(\beta, q)$ and $\left(\beta_{0}, \theta_{0}\right)$ denote the true values. First we obtain consistency of $\hat{\alpha}$ (and $\hat{\theta}$ ) under a strong metric, $\|\cdot\|_{s, \alpha}$ (and $\|\cdot\|_{s, \theta}$ ), and then establish that $\hat{\alpha}$ (and $\hat{\theta}$ ) converge to $\alpha_{0}$ (and $\theta_{0}$ ) at a rate faster than $n^{-1 / 4}$ under a weak metric, $\|\cdot\|_{\alpha}$ (and $\|\cdot\|_{\theta}$ ), which is necessary to obtain $\sqrt{n}$-asymptotic normality for $\hat{\beta}$. Our notations and results are built on Hu and Schennach (2008) and Ai and Chen (2003) (also see Chen 2007, Blundell, Chen, and Kristensen (2007), and Song 2012).

\subsection{Consistency}

Let $D_{t} \equiv\left(r_{t}, k_{t}^{*}, k_{t-1}^{*}, \mathbf{z}_{t}^{*}\right)$. Let the first smoothing parameter $\kappa_{1 n}=d_{\phi_{1}}+\kappa_{1 n_{\eta}}+\kappa_{1 n_{1}}+\kappa_{1 n_{2}}$ from the sieve approximation for $f_{1}$ and $f_{2}$ where $d_{\phi_{1}}=\operatorname{dim}\left(\Phi_{1}\right), \kappa_{1 n_{\eta}}=\operatorname{dim}\left(\mathcal{M}_{n}\right), \kappa_{1 n_{1}}=\operatorname{dim}\left(\mathcal{F}_{1 n}\right)$, and $\kappa_{1 n_{2}}=\operatorname{dim}\left(\mathcal{F}_{2 n}\right)$ and also let the second smoothing parameter $\kappa_{2 n}=\operatorname{dim}\left(\mathcal{H}_{n}\right)$ from the sieve approximation for $q(\cdot)$. Define $\|\theta\|_{s, \theta} \equiv\|\beta\|_{E}+\|q\|_{\infty, \nu},\|\alpha\|_{s, \alpha} \equiv\|\phi\|_{E}+\|\eta\|_{\infty, \nu}+\left\|f_{1}\right\|_{\infty, \nu}+\left\|f_{2}\right\|_{\infty, \nu}$, where $\|g\|_{\infty, \nu} \equiv \sup _{\xi}|g(\xi) \nu(\xi)|$ with a weight function $\nu(\xi)=\left(1+\|\xi\|_{E}^{2}\right)^{-\varsigma / 2}, \varsigma>\gamma_{1}>0$. Because

the supports of the mismeasured variables could be unbounded, we use the weighted sup-norm metric.

\section{Assumption 5.1.}

1. The data $\left\{D_{t i}=\left(r_{t, i}, k_{t, i}^{*}, k_{t-1, i}^{*}, \mathbf{z}_{t, i}^{*}\right): i=1, \ldots, n\right\}$ are $i . i . d$. for all $t=1, \ldots, T$

2. The density of $D_{t}, f_{D_{t}}$, satisfies $\int \nu\left(D_{t}\right)^{-2} f_{D_{t}}\left(D_{t}\right) d\left(D_{t}\right)<\infty$.

This assumption is about the nature of data. Next we assume $\alpha$ belong to a class of function that is well approximated by sieves.

\section{Assumption 5.2.}

1. There is a metric $\|\cdot\|_{s, \alpha}$ such that $\mathcal{A} \equiv \Psi \times \mathcal{F}_{1} \times \mathcal{F}_{2}$ is compact under $\|\cdot\|_{s, \alpha}$

2. For any $\alpha \in \mathcal{A}$, there exists $\Pi_{n} \alpha \in \mathcal{A}_{n} \equiv \Psi_{n} \times \mathcal{F}_{1 n} \times \mathcal{F}_{2 n}$ with $\Psi_{n} \equiv \Phi \times \mathcal{M}_{n}$ such that $\left\|\Pi_{n} \alpha-\alpha\right\|_{s, \alpha}=o(1)$.

We now impose an envelop condition and Hölder continuity on the log likelihood.

\section{Assumption 5.3.}


1. $E\left[\left|\ln f_{r_{t}, k_{t}^{*}, k_{t-1}^{*} \mid \mathbf{z}_{t}^{*}}\left(r_{t}, k_{t}^{*}, k_{t-1}^{*} \mid \mathbf{z}_{t}^{*}\right)\right|^{2}\right]$ is bounded

2. There exists a measurable function $h_{1}\left(D_{t}\right)$ with $E\left[\left|h_{1}\left(D_{t}\right)\right|^{2}\right]<\infty$ such that for any $\bar{\alpha}=$ $\left(\bar{\psi}, \bar{f}_{1}, \bar{f}_{2}\right)^{\prime} \in \mathcal{A}$,

$$
\left|\frac{f_{r_{t}, k_{t}^{*}, k_{t-1}^{*} \mid \mathbf{z}_{t}^{*}}^{|1|}\left(r_{t}, k_{t}^{*}, k_{t-1}^{*} \mid \mathbf{z}_{t}^{*} ; \bar{\alpha}, \nu\right)}{f_{r_{t}, k_{t}^{*}, k_{t-1}^{*} \mid \mathbf{z}_{t}^{*}}^{*}\left(r_{t}, k_{t}^{*}, k_{t-1}^{*} \mid \mathbf{z}_{t}^{*} ; \bar{\alpha}\right)}\right| \leq h_{1}\left(D_{t}\right),
$$

where the pathwise first derivative $f_{r_{t}, k_{t}^{*}, k_{t-1}^{*} \mid \mathbf{z}_{t}^{*}}\left(r_{t}, k_{t}^{*}, k_{t-1}^{*} \mid \mathbf{z}_{t}^{*} ; \bar{\alpha}, \nu\right)$ is defined in the proof of Theorem 5.10.1.

Assumption 5.4. $\kappa_{1 n} / n \rightarrow 0, \kappa_{2 n} / n \rightarrow 0$ and $\kappa_{1 n}, \kappa_{2 n} \rightarrow \infty$.

This is about rate conditions on the smoothing parameters for consistency. We next assume the parameter of interest $\theta$ is identified, which is satisfied under standard nonparametric rank conditions (e.g., completeness condition in Newey and Powell 2003).

Assumption 5.5. $\theta_{0} \in \Theta$ is the only $\theta \in \Theta$ satisfying $g\left(\mathbf{x}_{t} ; \theta\right)=0$.

We now assume a consistent estimator of the weighting function is available.

Assumption 5.6. 1. $\hat{A}\left(\mathbf{x}_{t}\right)=A\left(\mathbf{x}_{t}\right)+o_{p}(1)$ uniformly over $\mathbf{x}_{t} \in \mathcal{X}_{t} \equiv \operatorname{Supp}\left(\mathbf{x}_{t}\right) ; 2$. A( $\left.\mathbf{x}_{t}\right)$ is finite and strictly positive uniformly over $\mathbf{x}_{t} \in \mathcal{X}_{t}$.

Next we assume the moment function has bounded second moment and is continuous.

Assumption 5.7. 1. $E\left[\left\|g\left(\mathbf{x}_{t} ; \theta_{0}\right)\right\|^{2}\right]$ is bounded; 2. $g\left(\mathbf{x}_{t} ; \theta\right)$ is Hölder continuous in $\theta \in \Theta$.

We next restrict the parameter space, $\Theta$, and a metric, $\|\cdot\|_{s, \theta}$. We also define a sieve space used for approximating the parameter space.

\section{Assumption 5.8.}

1. There is a metric $\|\cdot\|_{s, \theta}$ such that $\Theta \equiv \mathcal{B} \times \mathcal{H}$ is compact under $\|\cdot\|_{s, \theta}$

2. For any $\theta \in \Theta$, there exists $\Pi_{n} \theta \in \Theta_{n} \equiv \mathcal{B} \times \mathcal{H}_{n}$ such that $\left\|\Pi_{n} \theta-\theta\right\|_{s, \theta}=o(1)$.

We also impose consistency of $\hat{f}_{k_{t-2}^{*}, k_{t-3}^{*}, m_{t}, l_{t}, m_{t-1}, l_{t-1}}$ and $\hat{f}_{m_{t}, l_{t}, m_{t-1}, l_{t-1}}$, which is well established in standard nonparametric density estimations (e.g. kernel or sieve estimation), required in steps to estimate $f_{k_{t}, k_{t-1} \mid m_{t}, l_{t}, m_{t-1}, l_{t-1}}$ and $f_{y_{t} \mid k_{t}, k_{t-1}, m_{t-1}, l_{t-1}}$.

\section{Assumption 5.9.}

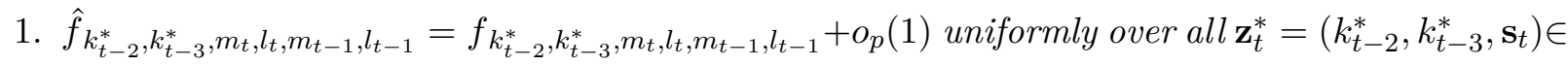
$\mathcal{Z}_{t}^{*} \equiv \operatorname{Supp}\left(\mathbf{z}_{t}^{*}\right)$ 
2. $\hat{f}_{m_{t}, l_{t}, m_{t-1}, l_{t-1}}=f_{m_{t}, l_{t}, m_{t-1}, l_{t-1}}+o_{p}(1)$ uniformly over all $\mathbf{s}_{t}=\left(m_{t}, l_{t}, m_{t-1}, l_{t-1}\right) \in \mathcal{S}_{t} \equiv$ $\operatorname{Supp}\left(\mathbf{s}_{t}\right)$.

We state consistency of the proposed estimators of $\alpha_{0}$ and $\theta_{0}$ in the following theorem.

\section{Theorem 5.10.}

1. Suppose Assumptions 4.1, 4.5 and 5.1-5.4 hold. Then $\left\|\hat{\alpha}_{n}-\alpha_{0}\right\|_{s, \alpha}=o_{p}(1)$.

2. Suppose Assumptions 4.1, 4.5 and 5.1-5.9 hold. Then $\left\|\hat{\theta}_{n}-\theta_{0}\right\|_{s, \theta}=o_{p}(1)$.

\subsection{Convergence Rates}

In this section, we establish $n^{-1 / 4}$ convergence rate of $\hat{\alpha}_{n}$ (and $\hat{\theta}_{n}$ ) under a weaker metric, $\|\cdot\|_{\alpha}$ (and $\|\cdot\|_{\theta}$ ), which is sufficient to obtain $\sqrt{n}$-asymptotic normality of $\hat{\beta}_{n}$ (and also $\hat{\phi}_{n}$ ). We need to introduce additional notations. Again our notations and results are closely related to $\mathrm{Hu}$ and Schennach (2008), Ai and Chen (2003), and Song (2012).

Denote the first pathwise derivative of $\ln f_{r_{t}, k_{t}^{*}, k_{t-1}^{*} \mid \mathbf{z}_{t}^{*}}\left(r_{t}, k_{t}^{*}, k_{t-1}^{*} \mid \mathbf{z}_{t}^{*} ; \alpha\right)$ at the direction $\left[\alpha-\alpha_{0}\right]$ evaluated at $\alpha_{0}$ by:

$$
\left.\frac{d \ln f_{r_{t}, k_{t}^{*}, k_{t-1}^{*} \mid \mathbf{z}_{t}^{*}}\left(r_{t}, k_{t}^{*}, k_{t-1}^{*} \mid \mathbf{z}_{t}^{*} ; \alpha_{0}\right)}{d \alpha}\left[\alpha-\alpha_{0}\right] \equiv \frac{d \ln f_{r_{t}, k_{t}^{*}, k_{t-1}^{*} \mid \mathbf{z}_{t}^{*}}\left(r_{t}, k_{t}^{*}, k_{t-1}^{*} \mid \mathbf{z}_{t}^{*} ;(1-\tau) \alpha_{0}+\tau \alpha\right)}{d \tau}\right|_{\tau=0}
$$

almost everywhere. Also for $\alpha_{1}, \alpha_{2} \in \mathcal{A}$ denote

$$
\begin{aligned}
\frac{d \ln f_{r_{t}, k_{t}^{*}, k_{t-1}^{*} \mid \mathbf{z}_{t}^{*}}\left(r_{t}, k_{t}^{*}, k_{t-1}^{*} \mid \mathbf{z}_{t}^{*} ; \alpha_{0}\right)}{d \alpha}\left[\alpha_{1}-\alpha_{2}\right]= & \frac{d \ln f_{r_{t}, k_{t}^{*}, k_{t-1}^{*} \mid \mathbf{z}_{t}^{*}}\left(r_{t}, k_{t}^{*}, k_{t-1}^{*} \mid \mathbf{z}_{t}^{*} ; \alpha_{0}\right)}{d \alpha}\left[\alpha_{1}-\alpha_{0}\right] \\
& -\frac{d \ln f_{r_{t}, k_{t}^{*}, k_{t-1}^{*} \mid \mathbf{z}_{t}^{*}}\left(r_{t}, k_{t}^{*}, k_{t-1}^{*} \mid \mathbf{z}_{t}^{*} ; \alpha_{0}\right)}{d \alpha}\left[\alpha_{2}-\alpha_{0}\right] .
\end{aligned}
$$

Then the pathwise derivative is written by:

$$
\begin{aligned}
& \frac{d \ln f_{r_{t}, k_{t}^{*}, k_{t-1}^{*} \mid \mathbf{z}_{t}^{*}}\left(r_{t}, k_{t}^{*}, k_{t-1}^{*} \mid \mathbf{z}_{t}^{*} ; \alpha_{0}\right)}{d \alpha}\left[\alpha-\alpha_{0}\right] \\
= & \frac{1}{f_{r_{t}, k_{t}^{*}, k_{t-1}^{*} \mid \mathbf{z}_{t}^{*}}\left(r_{t}, k_{t}^{*}, k_{t-1}^{*} \mid \mathbf{z}_{t}^{*} ; \alpha_{0}\right)} \\
& \times\left\{\int \frac{d}{d \psi} f_{r_{t} \mid \mathbf{z}_{t}}\left(r_{t} \mid \mathbf{z}_{t} ; \psi_{0}\right)\left[\psi-\psi_{0}\right] f_{k_{t}^{*}, k_{t-1}^{*} \mid \mathbf{z}_{t}}\left(k_{t}^{*}, k_{t-1}^{*} \mid \mathbf{z}_{t}\right) f_{k_{t}, k_{t-1} \mid \mathbf{z}_{t}^{*}}\left(k_{t}, k_{t-1} \mid \mathbf{z}_{t}^{*}\right) d k_{t} d k_{t-1}\right. \\
& +\int f_{r_{t} \mid \mathbf{z}_{t}}\left(r_{t} \mid \mathbf{z}_{t} ; \psi_{0}\right)\left[f_{1}\left(k_{t}^{*}, k_{t-1}^{*} \mid \mathbf{z}_{t}\right)-f_{k_{t}^{*}, k_{t-1}^{*} \mid \mathbf{z}_{t}}\left(k_{t}^{*}, k_{t-1}^{*} \mid \mathbf{z}_{t}\right)\right] f_{k_{t}, k_{t-1} \mid \mathbf{z}_{t}^{*}}\left(k_{t}, k_{t-1} \mid \mathbf{z}_{t}^{*}\right) d k_{t} d k_{t-1} \\
& \left.+\int f_{r_{t} \mid \mathbf{z}_{t}}\left(r_{t} \mid \mathbf{z}_{t} ; \psi_{0}\right) f_{k_{t}^{*}, k_{t-1}^{*} \mid \mathbf{z}_{t}}\left(k_{t}^{*}, k_{t-1}^{*} \mid \mathbf{z}_{t}\right)\left[f_{2}\left(k_{t}, k_{t-1} \mid \mathbf{z}_{t}^{*}\right)-f_{k_{t}, k_{t-1} \mid \mathbf{z}_{t}^{*}}\left(k_{t}, k_{t-1} \mid \mathbf{z}_{t}^{*}\right)\right] d k_{t} d k_{t-1}\right\} .
\end{aligned}
$$


For any $\alpha_{1}, \alpha_{2} \in \mathcal{A}$, the metric $\|\cdot\|_{\alpha}$ is defined as

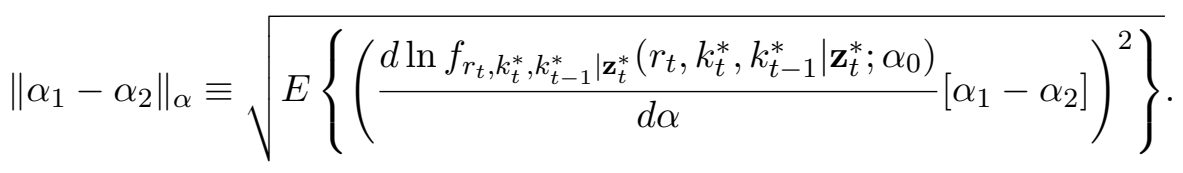

We state similar notations for $\theta$. Denote the first pathwise derivative of $\rho\left(\mathbf{y}_{t, t-1} ; \theta\right)$ at the direction $\left[\theta-\theta_{0}\right]$ evaluated at $\theta_{0}$ by:

$$
\left.\frac{d \rho\left(\mathbf{y}_{t, t-1} ; \theta_{0}\right)}{d \theta}\left[\theta-\theta_{0}\right] \equiv \frac{d \rho\left(\mathbf{y}_{t, t-1} ;(1-\tau) \theta_{0}+\tau \theta\right)}{d \tau}\right|_{\tau=0}
$$

almost everywhere and for any $\theta_{1}, \theta_{2} \in \Theta$ denote

$$
\begin{aligned}
\frac{d \rho\left(\mathbf{y}_{t, t-1} ; \theta_{0}\right)}{d \theta}\left[\theta_{1}-\theta_{2}\right] & \equiv \frac{d \rho\left(\mathbf{y}_{t, t-1} ; \theta_{0}\right)}{d \theta}\left[\theta_{1}-\theta_{0}\right]-\frac{d \rho\left(\mathbf{y}_{t, t-1} ; \theta_{0}\right)}{d \theta}\left[\theta_{2}-\theta_{0}\right] \\
\frac{d g\left(\mathbf{x}_{t} ; \theta_{0}\right)}{d \theta}\left[\theta_{1}-\theta_{2}\right] & \equiv\left(\begin{array}{c}
E\left\{\frac{d \rho_{1}\left(\mathbf{y}_{t} ; \beta_{0}\right)}{d \beta}\left[\beta_{1}-\beta_{2}\right] \mid \mathbf{x}_{t}\right\} \\
E\left\{\frac{d \rho_{2}\left(\mathbf{y}_{t, t-1} ; \theta_{0}\right)}{d \theta}\left[\theta_{1}-\theta_{2}\right] \mid \tilde{\mathbf{x}}_{t}\right\}
\end{array}\right) .
\end{aligned}
$$

Similarly, for any $\theta_{1}, \theta_{2} \in \Theta$, the metric $\|\cdot\|_{\theta}$ is defined as:

$$
\left\|\theta_{1}-\theta_{2}\right\|_{\theta} \equiv \sqrt{E\left\{\left(\frac{d g\left(\mathbf{x}_{t} ; \theta_{0}\right)}{d \theta}\left[\theta_{1}-\theta_{2}\right]\right)^{\prime} A\left(\mathbf{x}_{t}\right) \frac{d g\left(\mathbf{x}_{t} ; \theta_{0}\right)}{d \theta}\left[\theta_{1}-\theta_{2}\right]\right\}} .
$$

We now state sufficient conditions for the convergence rate of the proposed estimators of $\alpha_{0}$ and $\theta_{0}$. First we strengthen the requirement for the approximation order enough to obtain the convergence rate of estimators up to $o_{p}\left(n^{-1 / 4}\right)$. This is also well known to be satisfied when $\alpha$ and $\theta$ belong to a class of bounded and smooth functions.

\section{Assumption 5.11.}

1. $\ln f_{r_{t}, k_{t}^{*}, k_{t-1}^{*} \mid \mathbf{z}_{t}^{*}}\left(r_{t}, k_{t}^{*}, k_{t-1}^{*} \mid \mathbf{z}_{t}^{*} ; \alpha\right)$ satisfies an envelope condition in $\alpha \in \mathcal{A}_{n}$

2. $\ln f_{r_{t}, k_{t}^{*}, k_{t-1}^{*} \mid \mathbf{z}_{t}^{*}}\left(r_{t}, k_{t}^{*}, k_{t-1}^{*} \mid \mathbf{z}_{t}^{*} ; \alpha\right) \in \Lambda_{c}^{\mu_{1}, \nu}\left(\operatorname{Supp}\left(r_{t}, k_{t}^{*}, k_{t-1}^{*}, \mathbf{z}_{t}^{*}\right)\right)$ for some constant $c>0$ with $\mu_{1}>d_{D_{t}} / 2$, for all $\alpha \in \mathcal{A}_{n}$, where $d_{D_{t}}$ is the dimension of $D_{t}$.

This states an envelop condition and a smoothness condition on $\ln f_{r_{t}, k_{t}^{*}, k_{t-1}^{*} \mid \mathbf{z}_{t}^{*}}\left(r_{t}, k_{t}^{*}, k_{t-1}^{*} \mid \mathbf{z}_{t}^{*} ; \alpha\right)$. The next assumption quantifies the approximation error of $\Pi_{n} \alpha$ to $\alpha$, by the sieve.

Assumption 5.12. There is a constant $\mu_{1}>0$ such that for any $\alpha \in \mathcal{A}$, there exists $\Pi_{n} \alpha \in \mathcal{A}_{n}$ satisfying $\left\|\Pi_{n} \alpha-\alpha\right\|_{\alpha}=O\left(\kappa_{1 n}^{-\mu_{1}}\right)$, and $\kappa_{1 n}^{-\mu_{1}}=o\left(n^{-1 / 4}\right)$.

Similarly, next assumptions are imposed for estimating $\theta$ in the second stage. Assumption 5.14 below is well known to be satisfied for sieve approximations when $\Theta$ is a Hölder class. 


\section{Assumption 5.13.}

1. Each element of $\rho\left(\mathbf{y}_{t, t-1} ; \theta\right)$ satisfies an envelope condition in $\theta \in \Theta_{n}$

2. Each element of $g\left(\mathbf{x}_{t} ; \theta\right) \in \Lambda_{c}^{\mu_{2}, \nu}\left(\mathcal{X}_{t}\right)$ for some constant $c>0$ with $\mu_{2}>d_{\mathbf{x}_{t}} / 2$, for all $\theta \in \Theta_{n}$, where $d_{\mathbf{x}_{t}}$ is the dimension of $\mathbf{x}_{t}$.

Assumption 5.14. There is a constant $\mu_{2}>0$ such that for any $\theta \in \Theta$, there exists $\Pi_{n} \theta \in \Theta_{n}$ satisfying $\left\|\Pi_{n} \theta-\theta\right\|_{\theta}=O\left(\kappa_{2 n}^{-\mu_{2}}\right)$, and $\kappa_{2 n}^{-\mu_{2}}=o\left(n^{-1 / 4}\right)$.

Next assumption restricts the estimation error of the weight matrix.

Assumption 5.15. $\hat{A}\left(\mathbf{x}_{t}\right)=A\left(\mathbf{x}_{t}\right)+o_{p}\left(n^{-1 / 4}\right)$ uniformly over $\mathbf{x}_{t} \in \mathcal{X}_{t}$.

Define $\xi_{0 n} \equiv \sup _{\left(\xi_{11}, \xi_{12}, \xi_{13}\right) \in \operatorname{Supp}(\eta) \cup \operatorname{Supp}\left(k_{t}^{*}, k_{t-1}^{*}, \mathbf{z}_{t}\right) \cup \operatorname{Supp}\left(k_{t}, k_{t-1}, \mathbf{z}_{t}^{*}\right)}\left\|p^{\kappa_{n_{\eta}}}\left(\xi_{11}\right), p^{\kappa_{n_{1}}}\left(\xi_{12}\right), p^{\kappa_{n_{2}}}\left(\xi_{13}\right)\right\|_{E}$, which is non-decreasing in $\kappa_{1 n}$. Also $N\left(\delta_{1}, \mathcal{A}_{n},\|\cdot\|_{s, \alpha}\right)$ and $N\left(\delta_{2}, \Theta_{n},\|\cdot\|_{s, \theta}\right)$ define the minimal number of radius $\delta_{1}$ covering balls of $\mathcal{A}_{n}$ under the $\|\cdot\|_{s, \alpha}$ metric, and the minimal number of radius $\delta_{2}$ covering balls of $\Theta_{n}$ under the $\|\cdot\|_{s, \theta}$ metric, respectively. Two assumptions below impose conditions related to the sieve approximation in the first stage, such as size of sieve space, well-definedness of the norm, and the norm equivalence.

\section{Assumption 5.16.}

1. $\kappa_{1 n} \times \ln n \times \xi_{0 n}^{2} \times n^{-1 / 2}=o(1)$

2. $\ln \left[N\left(\delta_{1}, \mathcal{A}_{n},\|\cdot\|_{s, \alpha}\right)\right] \leq$ const. $\times \kappa_{1 n} \times \ln \left(\kappa_{1 n} / \delta_{1}\right)$.

\section{Assumption 5.17.}

1. $\mathcal{A}$ is convex in $\alpha_{0}$ and $f_{r_{t} \mid \mathbf{z}_{t}}\left(r_{t} \mid \mathbf{z}_{t} ; \psi\right)$ is pathwise differentiable at $\psi_{0}$

2. For some $c_{11}, c_{12}>0$,

$$
c_{11} E\left\{\ln \frac{f_{r_{t}, k_{t}^{*}, k_{t-1}^{*} \mid \mathbf{z}_{t}^{*}}\left(r_{t}, k_{t}^{*}, k_{t-1}^{*} \mid \mathbf{z}_{t}^{*} ; \alpha_{0}\right)}{f_{r_{t}, k_{t}^{*}, k_{t-1}^{*} \mid \mathbf{z}_{t}^{*}}\left(r_{t}, k_{t}^{*}, k_{t-1}^{*} \mid \mathbf{z}_{t}^{*} ; \alpha\right)}\right\} \leq\left\|\alpha-\alpha_{0}\right\|_{\alpha}^{2} \leq c_{12} E\left\{\ln \frac{f_{r_{t}, k_{t}^{*}, k_{t-1}^{*} \mid \mathbf{z}_{t}^{*}}\left(r_{t}, k_{t}^{*}, k_{t-1}^{*} \mid \mathbf{z}_{t}^{*} ; \alpha_{0}\right)}{f_{r_{t}, k_{t}^{*}, k_{t-1}^{*} \mid \mathbf{z}_{t}^{*}}\left(r_{t}, k_{t}^{*}, k_{t-1}^{*} \mid \mathbf{z}_{t}^{*} ; \alpha\right)}\right\}
$$

holds for all $\alpha_{1} \in \mathcal{A}_{n}$ with $\left\|\alpha-\alpha_{0}\right\|_{s, \alpha}=o(1)$.

Similarly, conditions associated with the sieve approximation in the second stage are imposed in the next two assumptions.

\section{Assumption 5.18.}

1. $\kappa_{2 n} \times \ln n \times \xi_{0 n}^{2} \times n^{-1 / 2}=o(1)$

2. $\ln \left[N\left(\delta_{2}, \Theta_{n},\|\cdot\|_{s, \theta}\right)\right] \leq$ const. $\times \kappa_{2 n} \times \ln \left(\kappa_{2 n} / \delta_{2}\right)$. 


\section{Assumption 5.19.}

1. $\Theta$ is convex in $\theta_{0}$ and $\rho\left(\mathbf{y}_{t, t-1} ; \theta\right)$ is pathwise differentiable at $\theta_{0}$

2. For some $c_{21}, c_{22}>0$,

$$
c_{21} E\left\{g\left(\mathbf{x}_{t} ; \theta\right)^{\prime} A\left(\mathbf{x}_{t}\right) g\left(\mathbf{x}_{t} ; \theta\right)\right\} \leq\left\|\theta-\theta_{0}\right\|_{\theta}^{2} \leq c_{22} E\left\{g\left(\mathbf{x}_{t} ; \theta\right)^{\prime} A\left(\mathbf{x}_{t}\right) g\left(\mathbf{x}_{t} ; \theta\right)\right\}
$$

holds for all $\theta \in \Theta_{n}$ with $\left\|\theta-\theta_{0}\right\|_{s, \theta}=o(1)$.

We next impose convergence rates of $\hat{f}_{k_{t-2}^{*}, k_{t-3}^{*}, m_{t}, l_{t}, m_{t-1}, l_{t-1}}$ and $\hat{f}_{m_{t}, l_{t}, m_{t-1}, l_{t-1}}$, which appear in (11) and (12).

\section{Assumption 5.20.}

1. $\hat{f}_{k_{t-2}^{*}, k_{t-3}^{*}, m_{t}, l_{t}, m_{t-1}, l_{t-1}}=f_{k_{t-2}^{*}, k_{t-3}^{*}, m_{t}, l_{t}, m_{t-1}, l_{t-1}}+o_{p}\left(n^{-1 / 4}\right)$ uniformly over all $\mathbf{z}_{t}^{*}=\left(k_{t-2}^{*}, k_{t-3}^{*}, \mathbf{s}_{t}\right)$ $\in \mathcal{Z}_{t}^{*}$

2. $\hat{f}_{m_{t}, l_{t}, m_{t-1}, l_{t-1}}=f_{m_{t}, l_{t}, m_{t-1}, l_{t-1}}+o_{p}\left(n^{-1 / 4}\right)$ uniformly over all $\mathbf{s}_{t}=\left(m_{t}, l_{t}, m_{t-1}, l_{t-1}\right) \in \mathcal{S}_{t}$.

Under these Assumptions we obtain the following convergence rate results in the weaker metrics.

\section{Theorem 5.21.}

1. Suppose Assumptions 4.1, 4.5, 5.1-5.4, 5.11-5.12, and 5.16-5.17 hold. Then $\left\|\hat{\alpha}_{n}-\alpha_{0}\right\|_{\alpha}=$ $o_{p}\left(n^{-1 / 4}\right)$.

2. Suppose Assumptions 4.1, 4.5, 5.1-5.9, and 5.11-5.20 hold. Then $\left\|\hat{\theta}_{n}-\theta_{0}\right\|_{\theta}=o_{p}\left(n^{-1 / 4}\right)$.

\section{Asymptotic Normality}

We now establish the asymptotic normality and $\sqrt{n}$ - consistency of $\hat{\phi}_{n}$ and $\hat{\beta}_{n}$. We adopt useful notations introduced in $\mathrm{Ai}$ and Chen (2003). Let $\overline{\mathbf{V}}_{1}, \overline{\mathbf{V}}_{2}$ denote the closure of the linear span of $\mathcal{A}-\alpha_{0}$ under the metric $\|\cdot\|_{\alpha}$ and the closure of the linear span of $\Theta-\theta_{0}$ under the metric $\|\cdot\|_{\theta}$, respectively. Note $\overline{\mathbf{V}}_{1}=\mathcal{R}^{d_{\phi}} \times \overline{\mathcal{W}}_{1}$ with $\overline{\mathcal{W}}_{1} \equiv \overline{\mathcal{M} \times \mathcal{F}_{1} \times \mathcal{F}_{2}}-\left\{\left(\eta_{0}, f_{k_{t}^{*}, k_{t-1}^{*} \mid \mathbf{z}_{t}}\left(k_{t}^{*}, k_{t-1}^{*} \mid \mathbf{z}_{t}\right), f_{k_{t}, k_{t-1} \mid \mathbf{z}_{t}^{*}}\left(k_{t}, k_{t-1} \mid \mathbf{z}_{t}^{*}\right)\right)^{\prime}\right\}$ and $\overline{\mathbf{V}}_{2}=\mathcal{R}^{d_{\beta}} \times \overline{\mathcal{W}}_{2}$ with $\overline{\mathcal{W}}_{2} \equiv \overline{\mathcal{H}}-\left\{q_{0}\right\}$. Then $\left(\overline{\mathbf{V}}_{1},\|\cdot\|_{\alpha}\right)$ becomes a Hilbert space with the inner product $\left\langle v_{11}, v_{12}\right\rangle_{\alpha}$ induced by $\|\cdot\|_{\alpha}$ :

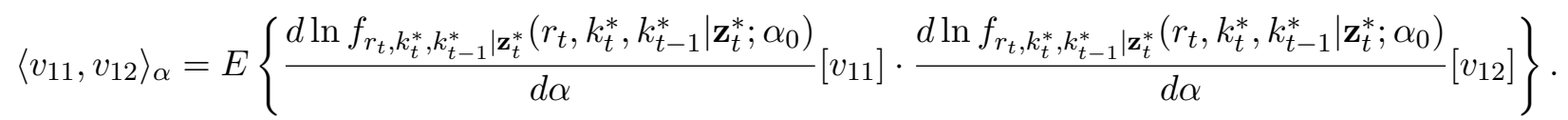

Also $\left(\overline{\mathbf{V}}_{2},\|\cdot\|_{\theta}\right)$ is a Hilbert space with the inner product:

$$
\left\langle v_{21}, v_{22}\right\rangle_{\theta}=E\left\{\left(\frac{d g\left(\mathbf{x}_{t} ; \theta_{0}\right)}{d \theta}\left[v_{21}\right]\right)^{\prime} A\left(\mathbf{x}_{t}\right) \frac{d g\left(\mathbf{x}_{t} ; \theta_{0}\right)}{d \theta}\left[v_{22}\right]\right\}
$$


The pathwise derivative at $\alpha_{0}$ is defined as

$$
\begin{aligned}
& \frac{d \ln f_{r_{t}, k_{t}^{*}, k_{t-1}^{*} \mid \mathbf{z}_{t}^{*}}\left(r_{t}, k_{t}^{*}, k_{t-1}^{*} \mid \mathbf{z}_{t}^{*} ; \alpha_{0}\right)}{d \alpha}\left[\alpha-\alpha_{0}\right] \\
= & \frac{d \ln f_{r_{t}, k_{t}^{*}, k_{t-1}^{*} \mid \mathbf{z}_{t}^{*}}\left(r_{t}, k_{t}^{*}, k_{t-1}^{*} \mid \mathbf{z}_{t}^{*} ; \alpha_{0}\right)}{d \phi^{\prime}}\left(\phi-\phi_{0}\right)+\frac{d \ln f_{r_{t}, k_{t}^{*}, k_{t-1}^{*} \mid \mathbf{z}_{t}^{*}}\left(r_{t}, k_{t}^{*}, k_{t-1}^{*} \mid \mathbf{z}_{t}^{*} ; \alpha_{0}\right)}{d \eta}\left[\eta-\eta_{0}\right] \\
& +\frac{d \ln f_{r_{t}, k_{t}^{*}, k_{t-1}^{*} \mid \mathbf{z}_{t}^{*}}\left(r_{t}, k_{t}^{*}, k_{t-1}^{*} \mid \mathbf{z}_{t}^{*} ; \alpha_{0}\right)}{d f_{1}}\left[f_{1}-f_{k_{t}^{*}, k_{t-1}^{*} \mid \mathbf{z}_{t}}\left(k_{t}^{*}, k_{t-1}^{*} \mid \mathbf{z}_{t}\right)\right] \\
& +\frac{d \ln f_{r_{t}, k_{t}^{*}, k_{t-1}^{*} \mid \mathbf{z}_{t}^{*}}\left(r_{t}, k_{t}^{*}, k_{t-1}^{*} \mid \mathbf{z}_{t}^{*} ; \alpha_{0}\right)}{d f_{2}}\left[f_{2}-f_{k_{t}, k_{t-1} \mid \mathbf{z}_{t}^{*}}\left(k_{t}, k_{t-1} \mid \mathbf{z}_{t}^{*}\right)\right]
\end{aligned}
$$

and the pathwise derivative at $\theta_{0}$ is defined as

$$
\frac{d g\left(\mathbf{x}_{t} ; \theta_{0}\right)}{d \theta}\left[\theta-\theta_{0}\right] \equiv \frac{d g\left(\mathbf{x}_{t} ; \theta_{0}\right)}{d \beta^{\prime}}\left[\beta-\beta_{0}\right]+\frac{d g\left(\mathbf{x}_{t} ; \theta_{0}\right)}{d q}\left[q-q_{0}\right]
$$

For each component $\phi_{j}$ of $\phi, j=1,2, \ldots, d_{\phi}$, we define $w_{1 j}^{*} \in \overline{\mathcal{W}}_{1}$ as

$$
\begin{aligned}
& w_{1 j}^{*} \equiv\left(\eta_{j}^{*}, f_{1 j}^{*}, f_{2 j}^{*}\right)^{\prime} \\
= & \arg \min _{\left(\eta, f_{1}, f_{2}\right)^{\prime} \in \overline{\mathcal{W}}_{1}} E\left\{\left(\frac{d \ln f_{r_{t}, k_{t}^{*}, k_{t-1}^{*} \mid \mathbf{z}_{t}^{*}}\left(r_{t}, k_{t}^{*}, k_{t-1}^{*} \mid \mathbf{z}_{t}^{*} ; \alpha_{0}\right)}{d \phi_{j}}-\frac{d \ln f_{r_{t}, k_{t}^{*}, k_{t-1}^{*} \mid \mathbf{z}_{t}^{*}}\left(r_{t}, k_{t}^{*}, k_{t-1}^{*} \mid \mathbf{z}_{t}^{*} ; \alpha_{0}\right)}{d \eta}\left[\eta_{j}\right]\right.\right. \\
& \left.\left.-\frac{d \ln f_{r_{t}, k_{t}^{*}, k_{t-1}^{*} \mid \mathbf{z}_{t}^{*}}\left(r_{t}, k_{t}^{*}, k_{t-1}^{*} \mid \mathbf{z}_{t}^{*} ; \alpha_{0}\right)}{d f_{1}}\left[f_{1 j}\right]-\frac{d \ln f_{r_{t}, k_{t}^{*}, k_{t-1}^{*} \mid \mathbf{z}_{t}^{*}}\left(r_{t}, k_{t}^{*}, k_{t-1}^{*} \mid \mathbf{z}_{t}^{*} ; \alpha_{0}\right)}{d f_{2}}\left[f_{2 j}\right]\right)^{2}\right\} .
\end{aligned}
$$

Also for each component $\beta_{j}$ of $\beta, j=1,2, \ldots, d_{\beta}$, we define $w_{2 j}^{*} \in \overline{\mathcal{W}}_{2}$ as

$$
=\arg \min _{w_{2 j} \in \overline{\mathcal{W}}_{2}} E\left\{\left(\frac{d g\left(\mathbf{x}_{t} ; \theta_{0}\right)}{d \beta_{j}}-\frac{d g\left(\mathbf{x}_{t} ; \theta_{0}\right)}{d q}\left[w_{2 j}\right]\right)^{\prime} A\left(\mathbf{x}_{t}\right)\left(\frac{d g\left(\mathbf{x}_{t} ; \theta_{0}\right)}{d \beta_{j}}-\frac{d g\left(\mathbf{x}_{t} ; \theta_{0}\right)}{d q}\left[w_{2 j}\right]\right)\right\} .
$$

We let $w_{1}^{*}=\left(w_{11}^{*}, w_{12}^{*}, \ldots, w_{1 d_{\phi}}^{*}\right)$ and define

$$
\begin{aligned}
& \frac{d \ln f_{r_{t}, k_{t}^{*}, k_{t-1}^{*} \mid \mathbf{z}_{t}^{*}}\left(r_{t}, k_{t}^{*}, k_{t-1}^{*} \mid \mathbf{z}_{t}^{*} ; \alpha_{0}\right)}{d f}\left[w_{1 j}^{*}\right] \\
= & \frac{d \ln f_{r_{t}, k_{t}^{*}, k_{t-1}^{*} \mid \mathbf{z}_{t}^{*}}\left(r_{t}, k_{t}^{*}, k_{t-1}^{*} \mid \mathbf{z}_{t}^{*} ; \alpha_{0}\right)}{d \eta}\left[\eta_{j}^{*}\right]+\frac{d \ln f_{r_{t}, k_{t}^{*}, k_{t-1}^{*} \mid \mathbf{z}_{t}^{*}}\left(r_{t}, k_{t}^{*}, k_{t-1}^{*} \mid \mathbf{z}_{t}^{*} ; \alpha_{0}\right)}{d f_{1}}\left[f_{1 j}^{*}\right] \\
& +\frac{d \ln f_{r_{t}, k_{t}^{*}, k_{t-1}^{*} \mid \mathbf{z}_{t}^{*}}\left(r_{t}, k_{t}^{*}, k_{t-1}^{*} \mid \mathbf{z}_{t}^{*} ; \alpha_{0}\right)}{d f_{2}}\left[f_{2 j}^{*}\right]
\end{aligned}
$$


and

$$
\begin{aligned}
& \frac{d \ln f_{r_{t}, k_{t}^{*}, k_{t-1}^{*} \mid \mathbf{z}_{t}^{*}}\left(r_{t}, k_{t}^{*}, k_{t-1}^{*} \mid \mathbf{z}_{t}^{*} ; \alpha_{0}\right)}{d f}\left[w_{1}^{*}\right] \\
= & \left(\frac{d \ln f_{r_{t}, k_{t}^{*}, k_{t-1}^{*} \mid \mathbf{z}_{t}^{*}}\left(r_{t}, k_{t}^{*}, k_{t-1}^{*} \mid \mathbf{z}_{t}^{*} ; \alpha_{0}\right)}{d f}\left[w_{11}^{*}\right], \ldots, \frac{d \ln f_{r_{t}, k_{t}^{*}, k_{t-1}^{*} \mid \mathbf{z}_{t}^{*}}\left(r_{t}, k_{t}^{*}, k_{t-1}^{*} \mid \mathbf{z}_{t}^{*} ; \alpha_{0}\right)}{d f}\left[w_{1 d_{\phi}}^{*}\right]\right) .
\end{aligned}
$$

Also let $w_{2}^{*}=\left(w_{21}^{*}, w_{22}^{*}, \ldots, w_{2 d_{\beta}}^{*}\right)$ and

$$
\frac{d g\left(\mathbf{x}_{t} ; \theta_{0}\right)}{d q}\left[w_{2}^{*}\right]=\left(\frac{d g\left(\mathbf{x}_{t} ; \theta_{0}\right)}{d q}\left[w_{21}^{*}\right], \ldots, \frac{d g\left(\mathbf{x}_{t} ; \theta_{0}\right)}{d q}\left[w_{2 d_{\beta}}^{*}\right]\right)
$$

We also define the row vectors

$$
\begin{aligned}
G_{w_{1}^{*}}\left(D_{t}, \alpha_{0}\right) & \equiv \frac{d \ln f_{r_{t}, k_{t}^{*}, k_{t-1}^{*} \mid \mathbf{z}_{t}^{*}}\left(r_{t}, k_{t}^{*}, k_{t-1}^{*} \mid \mathbf{z}_{t}^{*} ; \alpha_{0}\right)}{d \phi^{\prime}}-\frac{d \ln f_{r_{t}, k_{t}^{*}, k_{t-1}^{*} \mid \mathbf{z}_{t}^{*}}\left(r_{t}, k_{t}^{*}, k_{t-1}^{*} \mid \mathbf{z}_{t}^{*} ; \alpha_{0}\right)}{d f}\left[w_{1}^{*}\right], \\
G_{w_{2}^{*}}\left(\mathbf{x}_{t}, \theta_{0}\right) & \equiv \frac{d g\left(\mathbf{x}_{t} ; \theta_{0}\right)}{d \beta^{\prime}}-\frac{d g\left(\mathbf{x}_{t} ; \theta_{0}\right)}{d q}\left[w_{2}^{*}\right] .
\end{aligned}
$$

Define $s_{1}(\alpha) \equiv \lambda_{1}^{\prime} \phi$ for $\lambda_{1} \in \mathcal{R}^{d_{\phi}}, \lambda_{1} \neq 0$ and $s_{2}(\theta) \equiv \lambda_{2}^{\prime} \theta$ for $\lambda_{2} \in \mathcal{R}^{d_{\beta}}, \lambda_{2} \neq 0$. Let $J_{1}=$ $E\left[G_{w_{1}^{*}}\left(D_{t}, \alpha_{0}\right)^{\prime} G_{w_{1}^{*}}\left(D_{t}, \alpha_{0}\right)\right]$ and $J_{2}=E\left[G_{w_{2}^{*}}\left(\mathbf{x}_{t}, \theta_{0}\right)^{\prime} G_{w_{2}^{*}}\left(\mathbf{x}_{t}, \theta_{0}\right)\right]$. Then the Riesz representation theorem implies that there exist representers $v_{1}^{*}$ and $v_{2}^{*}$ such that

$$
s_{1}(\alpha)-s_{1}\left(\alpha_{0}\right) \equiv \lambda_{1}^{\prime}\left(\phi-\phi_{0}\right)=\left\langle v_{1}^{*}, \alpha-\alpha_{0}\right\rangle_{\alpha},
$$

for all $\alpha \in \mathcal{A}$ where $v_{1}^{*} \equiv\left(v_{\phi}^{*}, v_{f}^{*}\right) \in \overline{\mathbf{V}}_{1}, v_{\phi}^{*}=J_{1}^{-1} \lambda_{1}, v_{f}^{*}=-w_{1}^{*} \times v_{\phi}^{*}$ and that

$$
s_{2}(\theta)-s_{2}\left(\theta_{0}\right) \equiv \lambda_{2}^{\prime}\left(\theta-\theta_{0}\right)=\left\langle v_{2}^{*}, \theta-\theta_{0}\right\rangle_{\theta},
$$

for all $\theta \in \Theta$ where $v_{2}^{*} \equiv\left(v_{\beta}^{*}, v_{q}^{*}\right) \in \overline{\mathbf{V}}_{2}, v_{\beta}^{*}=J_{2}^{-1} \lambda_{2}, v_{q}^{*}=-w_{2}^{*} \times v_{\beta}^{*}$.

We denote, for any $v_{1} \in \overline{\mathbf{V}}_{1}$,

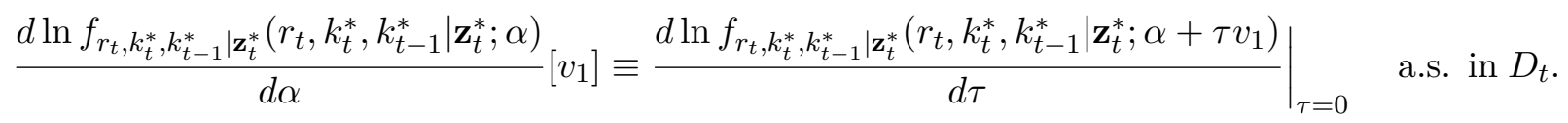

We also denote

$$
\left.\frac{d \rho\left(\mathbf{y}_{t, t-1} ; \theta\right)}{d \theta}\left[v_{2}\right] \equiv \frac{d \rho\left(\mathbf{y}_{t, t-1} ; \theta+\tau v_{2}\right)}{d \tau}\right|_{\tau=0} \quad \text { a.s. in } \mathbf{y}_{t, t-1}
$$

and

$$
\frac{d g\left(\mathbf{x}_{t} ; \theta\right)}{d \theta}\left[v_{2}\right] \equiv E\left\{\frac{d \rho\left(\mathbf{y}_{t, t-1} ; \theta\right)}{d \theta}\left[v_{2}\right] \mid \mathbf{x}_{t}\right\} \quad \text { a.s. in } \mathbf{x}_{t}
$$


for any $v_{2} \in \overline{\mathbf{V}}_{2}$.

Define $\mathcal{N}_{01 n}=\left\{\alpha: \alpha \in \mathcal{A}_{n},\left\|\alpha-\alpha_{0}\right\|_{\alpha}=o\left(n^{-1 / 4}\right),\left\|\alpha-\alpha_{0}\right\|_{s, \alpha}=o(1)\right\}$ and define $\mathcal{N}_{01}$ the same except $\mathcal{A}_{n}$ replaced with $\mathcal{A}$. We denote a local alternative $\alpha^{*}\left(\alpha, \varepsilon_{n}\right)=\left(1-\varepsilon_{n}\right) \alpha+\varepsilon_{n}\left(v_{1}^{*}+\alpha_{0}\right)$ with $\varepsilon_{n}=o\left(n^{-1 / 2}\right)$, for $\alpha \in \mathcal{N}_{01 n}$. Let $\Pi_{n} \alpha^{*}\left(\alpha, \varepsilon_{n}\right)$ be the projection of $\alpha^{*}\left(\alpha, \varepsilon_{n}\right)$ onto $\mathcal{A}_{n}$. Also define $\mathcal{N}_{02 n}=\left\{\theta: \theta \in \Theta_{n},\left\|\theta-\theta_{0}\right\|_{\theta}=o\left(n^{-1 / 4}\right),\left\|\theta-\theta_{0}\right\|_{s, \theta}=o(1)\right\}$ and define $\mathcal{N}_{02}$ the same except $\Theta_{n}$ replaced with $\Theta$.

We state the sufficient conditions for the $\sqrt{n}$-normality of $\hat{\phi_{n}}$ and $\hat{\beta_{n}}$.

\section{Assumption 6.1.}

1. $E\left[G_{w_{1}^{*}}\left(D_{t}, \alpha_{0}\right)^{\prime} G_{w_{1}^{*}}\left(D_{t}, \alpha_{0}\right)\right]$ exists, is bounded, and is positive-definite

2. $\phi_{0} \in \operatorname{int}(\Phi)$.

Assumption 6.2. There is $v_{1 n}^{*}=\left(v_{\phi}^{*},-\Pi_{n} w_{1}^{*} \times v_{\phi}^{*}\right) \in \mathcal{A}_{n}-\left\{\alpha_{0}\right\}$ such that $\left\|v_{1 n}^{*}-v_{1}^{*}\right\|_{\alpha}=o\left(n^{-1 / 4}\right)$.

Assumption 6.1 contains a standard rank condition and the bounded second moment condition that ensure the existence of the Riesz representation. Assumption 6.2 states that the Riesz representer is also well approximated by the sieves. Next we impose an envelope condition on the second derivative of the log likelihood, which is associated with the stochastic equicontinuity.

Assumption 6.3. There exists measurable function $h_{2}\left(D_{t}\right)$ with $E\left[\left(h_{2}\left(D_{t}\right)\right)^{2}\right]<\infty$ such that for any $\bar{\alpha}=\left(\bar{\psi}, \bar{f}_{1}, \bar{f}_{2}\right)^{\prime} \in \mathcal{N}_{01}$,

$$
\left|\frac{f_{r_{t}, k_{t}^{*}, k_{t-1}^{*} \mid \mathbf{z}_{t}^{*}}^{|1|}\left(r_{t}, k_{t}^{*}, k_{t-1}^{*} \mid \mathbf{z}_{t}^{*} ; \bar{\alpha}, \nu\right)}{f_{r_{t}, k_{t}^{*}, k_{t-1}^{*} \mid \mathbf{z}_{t}^{*}}\left(r_{t}, k_{t}^{*}, k_{t-1}^{*} \mid \mathbf{z}_{t}^{*} ; \bar{\alpha}\right)}\right|^{2}+\left|\frac{f_{r_{t}, k_{t}^{*}, k_{t-1}^{*} \mid \mathbf{z}_{t}^{*}}^{|2|}\left(r_{t}, k_{t}^{*}, k_{t-1}^{*} \mid \mathbf{z}_{t}^{*} ; \bar{\alpha}, \nu\right)}{f_{r_{t}, k_{t}^{*}, k_{t-1}^{*} \mid \mathbf{z}_{t}^{*}}\left(r_{t}, k_{t}^{*}, k_{t-1}^{*} \mid \mathbf{z}_{t}^{*} ; \bar{\alpha}\right)}\right| \leq h_{2}\left(D_{t}\right)
$$

where the pathwise first derivative $f_{r_{t}, k_{t}^{*}, k_{t-1}^{*} \mid \mathbf{z}_{t}^{*}}^{1 \mid}\left(r_{t}, k_{t}^{*}, k_{t-1}^{*} \mid \mathbf{z}_{t}^{*} ; \bar{\alpha}, \nu\right)$ and the pathwise second derivative $f_{r_{t}, k_{t}^{*}, k_{t-1}^{*} \mid \mathbf{z}_{t}^{*}}^{|2|}\left(r_{t}, k_{t}^{*}, k_{t-1}^{*} \mid \mathbf{z}_{t}^{*} ; \bar{\alpha}, \nu\right)$ are defined in the proofs of Theorem 5.10 .1 and Theorem 6.12.1, respectively.

We now introduce some notations for the assumptions below. Let

$$
\begin{aligned}
& \frac{d \ln f_{r_{t}, k_{t}^{*}, k_{t-1}^{*} \mid \mathbf{z}_{t}^{*}}\left(r_{t}, k_{t}^{*}, k_{t-1}^{*} \mid \mathbf{z}_{t}^{*} ; \alpha_{0}\right)}{d \alpha}\left[p^{\kappa_{1 n}}\right] \\
& =\left(\left(\frac{d \ln f_{r_{t}, k_{t}^{*}, k_{t-1}^{*} \mid \mathbf{z}_{t}^{*}}\left(r_{t}, k_{t}^{*}, k_{t-1}^{*} \mid \mathbf{z}_{t}^{*} ; \alpha_{0}\right)}{d \phi}\right)^{\prime},\left(\frac{d \ln f_{r_{t}, k_{t}^{*}, k_{t-1}^{*} \mid \mathbf{z}_{t}^{*}}\left(r_{t}, k_{t}^{*}, k_{t-1}^{*} \mid \mathbf{z}_{t}^{*} ; \alpha_{0}\right)}{d \eta}\left[p^{\kappa_{1 n}}\right]\right)^{\prime},\right. \\
& \left.\left(\frac{d \ln f_{r_{t}, k_{t}^{*}, k_{t-1}^{*} \mid \mathbf{z}_{t}^{*}}\left(r_{t}, k_{t}^{*}, k_{t-1}^{*} \mid \mathbf{z}_{t}^{*} ; \alpha_{0}\right)}{d f_{1}}\left[p^{\kappa_{1 n}}\right]\right)^{\prime},\left(\frac{d \ln f_{r_{t}, k_{t}^{*}, k_{t-1}^{*} \mid \mathbf{z}_{t}^{*}}\left(r_{t}, k_{t}^{*}, k_{t-1}^{*} \mid \mathbf{z}_{t}^{*} ; \alpha_{0}\right)}{d f_{2}}\left[p^{\kappa_{1 n}}\right]\right)^{\prime}\right)^{\prime},
\end{aligned}
$$


where for $\tilde{f}=\eta, f_{1}$, or $f_{2}$,

$$
\begin{aligned}
& \frac{d \ln f_{r_{t}, k_{t}^{*}, k_{t-1}^{*} \mid \mathbf{z}_{t}^{*}}\left(r_{t}, k_{t}^{*}, k_{t-1}^{*} \mid \mathbf{z}_{t}^{*} ; \alpha_{0}\right)}{d \tilde{f}}\left[p^{\kappa_{1 n}}\right]
\end{aligned}
$$

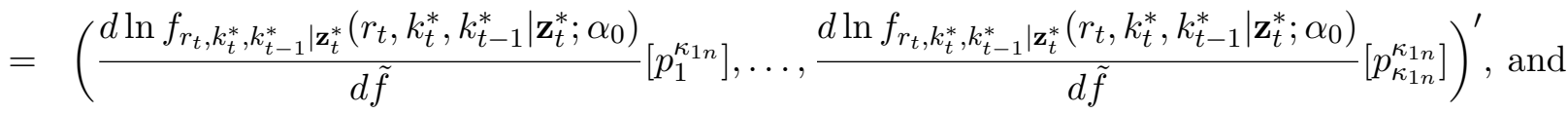

$$
\begin{aligned}
& \frac{d \ln f_{r_{t}, k_{t}^{*}, k_{t-1}^{*} \mid \mathbf{z}_{t}^{*}}\left(r_{t}, k_{t}^{*}, k_{t-1}^{*} \mid \mathbf{z}_{t}^{*} ; \alpha_{0}\right)}{d \phi} \\
& =\left(\frac{d \ln f_{r_{t}, k_{t}^{*}, k_{t-1}^{*} \mid \mathbf{z}_{t}^{*}}\left(r_{t}, k_{t}^{*}, k_{t-1}^{*} \mid \mathbf{z}_{t}^{*} ; \alpha_{0}\right)}{d \phi_{1}}, \ldots, \frac{d \ln f_{r_{t}, k_{t}^{*}, k_{t-1}^{*} \mid \mathbf{z}_{t}^{*}}\left(r_{t}, k_{t}^{*}, k_{t-1}^{*} \mid \mathbf{z}_{t}^{*} ; \alpha_{0}\right)}{d \phi_{d_{\phi}}}\right)^{\prime} .
\end{aligned}
$$

Let

$\Omega_{\kappa_{1 n}}=E\left\{\left(\frac{d \ln f_{r_{t}, k_{t}^{*}, k_{t-1}^{*} \mid \mathbf{z}_{t}^{*}}\left(r_{t}, k_{t}^{*}, k_{t-1}^{*} \mid \mathbf{z}_{t}^{*} ; \alpha_{0}\right)}{d \alpha}\left[p^{\kappa_{1 n}}\right]\right)\left(\frac{d \ln f_{r_{t}, k_{t}^{*}, k_{t-1}^{*} \mid \mathbf{z}_{t}^{*}}\left(r_{t}, k_{t}^{*}, k_{t-1}^{*} \mid \mathbf{z}_{t}^{*} ; \alpha_{0}\right)}{d \alpha}\left[p^{\kappa_{1 n}}\right]\right)^{\prime}\right\}$.

We now impose a normalization on the approximating functions, which trivially holds for e.g. orthonormalized approximating basis functions.

Assumption 6.4. The smallest eigenvalue of the matrices $\Omega_{\kappa_{1 n}}$ is bounded away from zero, and $\left\|p_{j}^{\kappa_{1 n}}\right\|_{\infty, \nu}<\infty$ for $j=1,2, \ldots, \kappa_{1 n}$ uniformly in $\kappa_{1 n}$.

Next we impose Lipschitz conditions on the fourth derivatives of the log densities.

Assumption 6.5. For all $\alpha \in \mathcal{N}_{01 n}$, there exists a measurable function $h_{3}\left(D_{t}\right)$ with $E\left[\left|h_{3}\left(D_{t}\right)\right|\right]<$ $\infty$ such that

$$
\left|\frac{d^{4}}{d \tau^{4}} \ln f_{r_{t}, k_{t}^{*}, k_{t-1}^{*} \mid \mathbf{z}_{t}^{*}}\left(r_{t}, k_{t}^{*}, k_{t-1}^{*} \mid \mathbf{z}_{t}^{*} ; \bar{\alpha}+\tau\left(\alpha-\alpha_{0}\right)\right)\right|_{\tau=0} \leq h_{3}\left(D_{t}\right)\left\|\alpha-\alpha_{0}\right\|_{s, \alpha}^{4} .
$$

Now we add conditions on the moment function. First, we impose a local identification condition for $\beta_{0}$. This also ensures the existence of the Riesz representer.

\section{Assumption 6.6.}

1. $E\left[G_{w_{2}^{*}}\left(\mathbf{x}_{t}, \theta_{0}\right)^{\prime} A\left(\mathbf{x}_{t}\right) G_{w_{2}^{*}}\left(\mathbf{x}_{t}, \theta_{0}\right)\right]$ exists, is bounded, and is positive-definite

2. $\beta_{0} \in \operatorname{int}(\mathcal{B})$

3. $\Sigma_{0}\left(\mathbf{x}_{t}\right) \equiv \operatorname{var}\left[\rho\left(\mathbf{y}_{t, t-1} ; \theta_{0}\right) \mid \mathbf{x}_{t}\right]$ is positive-definite for all $\mathbf{x}_{t} \in \mathcal{X}_{t}$.

Assumption 6.7. There is $v_{2 n}^{*}=\left(v_{\beta}^{*},-\Pi_{n} w_{2}^{*} \times v_{\beta}^{*}\right) \in \Theta_{n}-\left\{\theta_{0}\right\}$ such that $\left\|v_{2 n}^{*}-v_{2}^{*}\right\|_{\theta}=o\left(n^{-1 / 4}\right)$. This condition is required to ensure that the sieve space $\mathcal{H}_{n}$ approximates the space $\overline{\mathcal{H}}-\left\{q_{0}\right\}$, so the Riesz representer is also well approximated by the sieves. Now we impose an envelope condition for $\rho\left(\mathbf{y}_{t, t-1} ; \theta\right)$. 


\section{Assumption 6.8.}

1. For all $\theta \in \mathcal{N}_{02}$, the pathwise first derivative $\left(d \rho\left(\mathbf{y}_{t, t-1} ; \theta\right) / d \theta\right)\left[v_{2}\right]$ exists a.s. $\mathbf{y}_{t, t-1} \in \mathcal{Y}_{t}$

2. Each element of $\left(d \rho\left(\mathbf{y}_{t, t-1} ; \theta\right) / d \theta\right)\left[v_{2 n}^{*}\right]$ satisfies an envelope condition and is Hölder continuous in $\theta \in \mathcal{N}_{02 n}$

3. Each element of $\left(d g\left(\mathbf{x}_{t} ; \theta\right) / d \theta\right)\left[v_{2 n}^{*}\right]$ is in $\Lambda_{c}^{\gamma}\left(\mathcal{X}_{t}\right), \gamma>d_{x} / 2$ for all $\alpha \in \mathcal{N}_{02}$.

Next two assumptions are necessary to control the asymptotic bias when $\rho$ is a highly nonlinear function of $\theta$.

Assumption 6.9. Uniformly over $\theta \in \mathcal{N}_{02 n}$, we have

$$
E\left(\left\|\frac{d g\left(\mathbf{x}_{t} ; \theta\right)}{d \theta}\left[v_{2 n}^{*}\right]-\frac{d g\left(\mathbf{x}_{t} ; \theta_{0}\right)}{d \theta}\left[v_{2 n}^{*}\right]\right\|_{E}^{2}\right)=o\left(n^{-1 / 2}\right) .
$$

Additionally, we impose the following assumption.

Assumption 6.10. Uniformly over $\theta \in \mathcal{N}_{02}, \bar{\theta} \in \mathcal{N}_{02 n}$, we have

$$
E\left(\left\{\frac{d g\left(\mathbf{x}_{t} ; \theta_{0}\right)}{d \theta}\left[v_{2}^{*}\right]\right\} A\left(\mathbf{x}_{t}\right)\left\{\frac{d g\left(\mathbf{x}_{t} ; \theta\right)}{d \theta}\left[\bar{\theta}-\theta_{0}\right]-\frac{d g\left(\mathbf{x}_{t} ; \theta_{0}\right)}{d \theta}\left[\bar{\theta}-\theta_{0}\right]\right\}\right)=o\left(n^{-1 / 2}\right) .
$$

The following condition is required to have the higher order terms asymptotically negligible in the stochastic expansions to obtain the asymptotic normality.

Assumption 6.11. For all $\theta \in \mathcal{N}_{02 n}$, the pathwise second derivative $d^{2} \rho\left(\mathbf{y}_{t, t-1} ; \theta+\tau v_{2 n}^{*}\right) /\left.d \tau^{2}\right|_{\tau=0}$ exists a.s. $\mathbf{y}_{t, t-1} \in \mathcal{Y}_{t}$, and is bounded by a measurable function $c\left(\mathbf{y}_{t, t-1}\right)$ with $E\left[c\left(\mathbf{y}_{t, t-1}\right)^{2}\right]<\infty$.

We now conclude the $\sqrt{n}$-normality of $\left(\hat{\beta}_{n}, \hat{\phi}_{n}\right)$ in the following theorem.

\section{Theorem 6.12.}

1. Suppose Assumptions 4.1, 4.5, 5.1-5.4.1, 5.11-5.12, 5.16-5.17, and 6.1-6.5 hold. Then we have $\sqrt{n}\left(\hat{\phi}_{n}-\phi_{0}\right) \stackrel{d}{\longrightarrow} N\left(0, J_{1}^{-1}\right)$, where $J_{1}=E\left[G_{w_{1}^{*}}\left(D_{t}, \alpha_{0}\right)^{\prime} G_{w_{1}^{*}}\left(D_{t}, \alpha_{0}\right)\right]$.

2. Suppose Assumptions 4.1, 4.5, 5.1-5.9, 5.11-5.20, and 6.1-6.11 hold. Then $\sqrt{n}\left(\hat{\beta}_{n}-\beta_{0}\right) \stackrel{d}{\longrightarrow}$ $N\left(0, J_{2}^{-1}\right)$, where

$$
\begin{aligned}
J_{2}= & E\left[G_{w_{2}^{*}}\left(\mathbf{x}_{t}, \theta_{0}\right)^{\prime} A\left(\mathbf{x}_{t}\right) G_{w_{2}^{*}}\left(\mathbf{x}_{t}, \theta_{0}\right)\right] \times\left(E\left[G_{w_{2}^{*}}\left(\mathbf{x}_{t}, \theta_{0}\right)^{\prime} A\left(\mathbf{x}_{t}\right) \Sigma_{0}\left(\mathbf{x}_{t}\right) A\left(\mathbf{x}_{t}\right) G_{w_{2}^{*}}\left(\mathbf{x}_{t}, \theta_{0}\right)\right]\right)^{-1} \\
& \times E\left[G_{w_{2}^{*}}\left(\mathbf{x}_{t}, \theta_{0}\right)^{\prime} A\left(\mathbf{x}_{t}\right) G_{w_{2}^{*}}\left(\mathbf{x}_{t}, \theta_{0}\right)\right] .
\end{aligned}
$$

As in Ai and Chen (2003) one can also obtain an efficient two-step estimator of $\beta_{0}$ by using the optimal weight matrix as $A\left(\mathbf{x}_{t}\right)=\Sigma_{0}\left(\mathbf{x}_{t}\right)^{-1}$. In this case the asymptotic variance reduces to $J_{2}^{-1}=\left\{E\left[G_{w_{2}^{*}}\left(\mathbf{x}_{t}, \theta_{0}\right)^{\prime} \Sigma_{0}\left(\mathbf{x}_{t}\right)^{-1} G_{w_{2}^{*}}\left(\mathbf{x}_{t}, \theta_{0}\right)\right]\right\}^{-1}$. 
This asymptotic normality results show that in our estimation pre-stage estimations of the conditional mean functions via the conditional density functions do not contribute to the asymptotic distribution of $\hat{\beta}_{n}$. Thus estimation of the conditional mean functions or the conditional density functions satisfies the asymptotic independence with $\hat{\beta}_{n}$. This is indeed the same insight we find in Ai and Chen (2003) that the estimation of the conditional moment functions does not contribute to the asymptotic distribution of their sieve MD estimator.

\section{Conclusion}

The control function estimators for production functions of Olley and Pakes (1996) and Levinsohn and Petrin (2003) are not consistent if the arguments that enter the control function for productivity are measured with error. The problem is complicated by the fact that the unobserved productivity control function is an unknown non-linear function of capital and other inputs, so standard instrumental variable techniques cannot be employed. We combine results from the nonclassical measurement error literature (e.g. Hu and Schennach 2008) with a sieve minimum distance estimation techniques to recover the production function parameters (e.g. Ai and Chen 2003, Chen 2007, Song 2012) . We develop the asymptotic distribution for our estimator, which accounts for the multiple stages and the infinite-dimensional nuisance parameters. 


\section{Appendix}

\section{A Identification of Conditional Densities: Proof of Theorem 4.2}

We first define integral operators. $L_{R_{1}, R_{2} \mid R_{3}, R_{4}, R_{5}}$ denote an integral operator mapping $g \in \operatorname{Supp}\left(r_{3}, r_{4}\right)$ to $L_{R_{1}, R_{2} \mid R_{3}, R_{4}, R_{5}} g \in \operatorname{Supp}\left(r_{1}, r_{2}\right)$ for a given $R_{5}=r_{5}$ :

$$
\left[L_{R_{1}, R_{2} \mid R_{3}, R_{4}, R_{5}} g\right]\left(r_{1}, r_{2}\right) \equiv \int f_{R_{1}, R_{2} \mid R_{3}, R_{4}, R_{5}}\left(r_{1}, r_{2} \mid r_{3}, r_{4}, r_{5}\right) g\left(r_{3}, r_{4}\right) d r_{3} d r_{4}
$$

In addition, $\triangle_{R_{1} \mid R_{2}, R_{3}, R_{4}}$ denotes a diagonal operator mapping $g \in \operatorname{Supp}\left(r_{2}, r_{3}\right)$ to $\triangle_{R_{1} \mid R_{2}, R_{3}, R_{4}} g \in$ $\operatorname{Supp}\left(r_{2}, r_{3}\right)$ for a given $R_{4}=r_{4}$ :

$$
\triangle_{R_{1} \mid R_{2}, R_{3}, R_{4}} g \equiv f_{R_{1} \mid R_{2}, R_{3}, R_{4}}\left(r_{1} \mid r_{2}, r_{3}, r_{4}\right) g\left(r_{2}, r_{3}\right)
$$

Proof. Recall that $r_{t}=y_{t}$ and $\mathbf{s}_{t}=\left(m_{t}, l_{t}, m_{t-1}, l_{t-1}\right)$ or $r_{t} \in\left\{l_{t}, m_{t}\right\}$ and $\mathbf{s}_{t}=\left(m_{t-1}, l_{t-1}\right)$. We have

$$
\begin{aligned}
f_{r_{t}, k_{t}^{*}, k_{t-1}^{*} \mid k_{t-2}^{*}, k_{t-3}^{*}, \mathbf{s}_{t}} & =\int f_{r_{t}, k_{t}, k_{t-1}, k_{t}^{*}, k_{t-1}^{*} \mid k_{t-2}^{*}, k_{t-3}^{*}, \mathbf{s}_{t}} d k_{t} d k_{t-1} \\
& =\int f_{r_{t} \mid k_{t}, k_{t-1}, k_{t}^{*}, k_{t-1}^{*}, k_{t-2}^{*}, k_{t-3}^{*}, \mathbf{s}_{t}} f_{k_{t}, k_{t-1}, k_{t}^{*}, k_{t-1}^{*} \mid k_{t-2}^{*}, k_{t-3}^{*}, \mathbf{s}_{t}} d k_{t} d k_{t-1} \\
& =\int f_{r_{t} \mid k_{t}, k_{t-1}, \mathbf{s}_{t}} f_{k_{t}^{*}, k_{t-1}^{*} \mid k_{t}, k_{t-1}, k_{t-2}^{*}, k_{t-3}^{*}, \mathbf{s}_{t}} f_{k_{t}, k_{t-1} \mid k_{t-2}^{*}, k_{t-3}^{*}, \mathbf{s}_{t}} d k_{t} d k_{t-1} \\
& =\int f_{r_{t} \mid k_{t}, k_{t-1}, \mathbf{s}_{t}} f_{k_{t}^{*}, k_{t-1}^{*} \mid k_{t}, k_{t-1}, \mathbf{s}_{t}} f_{k_{t}, k_{t-1} \mid k_{t-2}^{*}, k_{t-3}^{*}, \mathbf{s}_{t}} d k_{t} d k_{t-1}
\end{aligned}
$$

by Assumption 4.1.1. Now we need to show uniqueness of the decomposition. Using operator notation, we have

$$
\begin{aligned}
& {\left[L_{r_{t}, k_{t}^{*}, k_{t-1}^{*} \mid k_{t-2}^{*}, k_{t-3}^{*}, \mathbf{s}_{t}} g\right]\left(k_{t}^{*}, k_{t-1}^{*}\right)} \\
& =\int f_{r_{t}, k_{t}^{*}, k_{t-1}^{*} \mid k_{t-2}^{*}, k_{t-3}^{*}, \mathbf{s}_{t}} g\left(k_{t-2}^{*}, k_{t-3}^{*}\right) d k_{t-2}^{*} d k_{t-3}^{*} \\
& =\iint f_{r_{t} \mid k_{t}, k_{t-1}, \mathbf{s}_{t}} f_{k_{t}^{*}, k_{t-1}^{*} \mid k_{t}, k_{t-1}, \mathbf{s}_{t}} f_{k_{t}, k_{t-1} \mid k_{t-2}^{*}, k_{t-3}^{*}, \mathbf{s}_{t}} d k_{t} d k_{t-1} g\left(k_{t-2}^{*}, k_{t-3}^{*}\right) d k_{t-2}^{*} d k_{t-3}^{*} \\
& =\int f_{k_{t}^{*}, k_{t-1}^{*} \mid k_{t}, k_{t-1}, \mathbf{s}_{t}} f_{r_{t} \mid k_{t}, k_{t-1}, \mathbf{s}_{t}} \int f_{k_{t}, k_{t-1} \mid k_{t-2}^{*}, k_{t-3}^{*}, \mathbf{s}_{t}} g\left(k_{t-2}^{*}, k_{t-3}^{*}\right) d k_{t-2}^{*} d k_{t-3}^{*} d k_{t} d k_{t-1} \\
& =\int f_{k_{t}^{*}, k_{t-1}^{*} \mid k_{t}, k_{t-1}, \mathbf{s}_{t}} f_{r_{t} \mid k_{t}, k_{t-1}, \mathbf{s}_{t}}\left[L_{k_{t}, k_{t-1} \mid k_{t-2}^{*}, k_{t-3}^{*}, \mathbf{s}_{t}} g\right]\left(k_{t}, k_{t-1}\right) d k_{t} d k_{t-1} \\
& =\int f_{k_{t}^{*}, k_{t-1}^{*} \mid k_{t}, k_{t-1}, \mathbf{s}_{t}}\left[\triangle_{r_{t} \mid k_{t}, k_{t-1}, \mathbf{s}_{t}} L_{k_{t}, k_{t-1} \mid k_{t-2}^{*}, k_{t-3}^{*}, \mathbf{s}_{t}} g\right]\left(k_{t}, k_{t-1}\right) d k_{t} d k_{t-1} \\
& =\left[L_{k_{t}^{*}, k_{t-1}^{*} \mid k_{t}, k_{t-1}, \mathbf{s}_{t}} \triangle_{r_{t} \mid k_{t}, k_{t-1}, \mathbf{s}_{t}} L_{k_{t}, k_{t-1} \mid k_{t-2}^{*}, k_{t-3}^{*}, \mathbf{s}_{t}} g\right]\left(k_{t}^{*}, k_{t-1}^{*}\right) \text {. }
\end{aligned}
$$


Thus we have

$$
L_{r_{t}, k_{t}^{*}, k_{t-1}^{*} \mid k_{t-2}^{*}, k_{t-3}^{*}, \mathbf{s}_{t}}=L_{k_{t}^{*}, k_{t-1}^{*} \mid k_{t}, k_{t-1}, \mathbf{s}_{t}} \triangle_{r_{t} \mid k_{t}, k_{t-1}, \mathbf{s}_{t}} L_{k_{t}, k_{t-1} \mid k_{t-2}^{*}, k_{t-3}^{*}, \mathbf{s}_{t}} .
$$

From the integration of the above equation over all $r_{t}$, we have

$$
L_{k_{t}^{*}, k_{t-1}^{*} \mid k_{t-2}^{*}, k_{t-3}^{*}, \mathbf{s}_{t}}=L_{k_{t}^{*}, k_{t-1}^{*} \mid k_{t}, k_{t-1}, \mathbf{s}_{t}} L_{k_{t}, k_{t-1} \mid k_{t-2}^{*}, k_{t-3}^{*}, \mathbf{s}_{t}} \cdot
$$

Then

$$
L_{k_{t}, k_{t-1} \mid k_{t-2}^{*}, k_{t-3}^{*}, \mathbf{s}_{t}}=L_{k_{t}^{*}, k_{t-1}^{*} \mid k_{t}, k_{t-1}, \mathbf{s}_{t}}^{-1} L_{k_{t}^{*}, k_{t-1}^{*} \mid k_{t-2}^{*}, k_{t-3}^{*}, \mathbf{s}_{t}},
$$

since $L_{k_{t}^{*}, k_{t-1}^{*} \mid k_{t}, k_{t-1}, \mathbf{s} t}$ is one-to-one. Since $L_{k_{t}^{*}, k_{t-1}^{*} \mid k_{t-2}^{*}, k_{t-3}^{*}, \mathbf{s}_{t}}$ is also one-to-one, rearranging two equations above gives

$$
L_{r_{t}, k_{t}^{*}, k_{t-1}^{*} \mid k_{t-2}^{*}, k_{t-3}^{*}, \mathbf{s}_{t}} L_{k_{t}^{*}, k_{t-1}^{*} \mid k_{t-2}^{*}, k_{t-3}^{*}, \mathbf{s}_{t}}^{-1}=L_{k_{t}^{*}, k_{t-1}^{*} \mid k_{t}, k_{t-1}, \mathbf{s}_{t}} \triangle_{r_{t}} \mid k_{t}, k_{t-1}, \mathbf{s}_{t} L_{k_{t}^{*}, k_{t-1}^{*} \mid k_{t}, k_{t-1}, \mathbf{s}_{t}}^{-1}
$$

Thus the observed operator on the left hand side has the form of an eigenvalue-eigenfunction decomposition, with the eigenvalues corresponding to the density $f_{r_{t} \mid k_{t}, k_{t-1}, \mathbf{s}_{t}}$ and the eigenfunctions corresponding to the density $f_{k_{t}^{*}, k_{t-1}^{*} \mid k_{t}, k_{t-1}, \mathbf{s}_{t}}$.

Assumption 4.1 ensures that this decomposition is unique, by a similar argument in $\mathrm{Hu}$ and Schennach (2008). Given the identification of $f_{r_{t} \mid k_{t}, k_{t-1}, \mathbf{s}_{t}}$ and $f_{k_{t}^{*}, k_{t-1}^{*} \mid k_{t}, k_{t-1}, \mathbf{s}_{t}}, f_{k_{t}, k_{t-1} \mid k_{t-2}^{*}, k_{t-3}^{*}, \mathbf{s}_{t}}$ is also identified from

$$
L_{k_{t}, k_{t-1} \mid k_{t-2}^{*}, k_{t-3}^{*}, \mathbf{s}_{t}}=L_{k_{t}^{*}, k_{t-1}^{*} \mid k_{t}, k_{t-1}, \mathbf{s}_{t}}^{-1} L_{k_{t}^{*}, k_{t-1}^{*} \mid k_{t-2}^{*}, k_{t-3}^{*}, \mathbf{s}_{t}}
$$

where $L_{k_{t}^{*}, k_{t-1}^{*} \mid k_{t-2}^{*}, k_{t-3}^{*}, \mathbf{s}_{t}}$ is observable.

\section{B Alternative Identification of $f_{l_{t} \mid k_{t}, k_{t-1}, m_{t-1}, l_{t-1}}$ and $f_{m_{t} \mid k_{t}, k_{t-1}, m_{t-1}, l_{t-1}}$}

We consider an alternative way of identifying $f_{l_{t} \mid k_{t}, k_{t-1}, m_{t-1}, l_{t-1}}$ and $f_{m_{t} \mid k_{t}, k_{t-1}, m_{t-1}, l_{t-1}}$. We show that they can be derived from

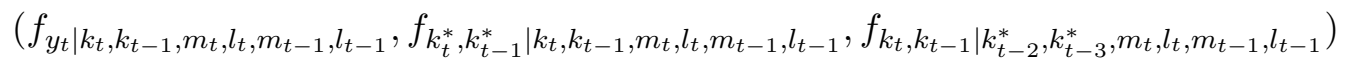

which are the objects identified as the results of Theorem 4.2. One advantage of this approach is that we need only one sieve MLE for (13). Others can be derived from these densities. On the other hand, it requires density estimation associated with inverse of linear operators. We first assume

\section{Assumption B.1.}

1. $f_{l_{t} \mid k_{t}, k_{t-1}, k_{t-2}^{*}, k_{t-3}^{*}, m_{t-1}, l_{t-1}}=f_{l_{t} \mid k_{t}, k_{t-1}, m_{t-1}, l_{t-1}}$

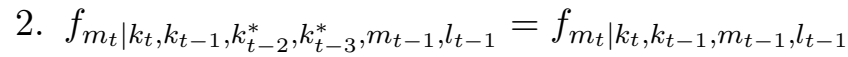


3. The operator $L_{k_{t}, k_{t-1} \mid k_{t-2}^{*}, k_{t-3}^{*}, m_{t-1}, l_{t-1}}$ is one-to-one.

We have from Assumption B.1.1 that,

$$
\begin{aligned}
f_{l_{t} \mid k_{t-2}^{*}, k_{t-3}^{*}, m_{t-1}, l_{t-1}} & =\int f_{l_{t} \mid k_{t}, k_{t-1}, k_{t-2}^{*}, k_{t-3}^{*}, m_{t-1}, l_{t-1}} f_{k_{t}, k_{t-1} \mid k_{t-2}^{*}, k_{t-3}^{*}, m_{t-1}, l_{t-1}} d k_{t} d k_{t-1} \\
& =\int f_{l_{t} \mid k_{t}, k_{t-1}, m_{t-1}, l_{t-1}} f_{k_{t}, k_{t-1} \mid k_{t-2}^{*}, k_{t-3}^{*}, m_{t-1}, l_{t-1}} d k_{t} d k_{t-1}
\end{aligned}
$$

which, in operator notation, is $L_{l_{t} \mid k_{t-2}^{*}, k_{t-3}^{*}, m_{t-1}, l_{t-1}}=L_{l_{t} \mid k_{t}, k_{t-1}, m_{t-1}, l_{t-1}} L_{k_{t}, k_{t-1} \mid k_{t-2}^{*}, k_{t-3}^{*}, m_{t-1}, l_{t-1}}$. Then, from Assumption B.1.3, we have that

$$
L_{l_{t} \mid k_{t}, k_{t-1}, m_{t-1}, l_{t-1}}=L_{l_{t} \mid k_{t-2}^{*}, k_{t-3}^{*}, m_{t-1}, l_{t-1}} L_{k_{t}, k_{t-1} \mid k_{t-2}^{*}, k_{t-3}^{*}, m_{t-1}, l_{t-1}} .
$$

Further note we identify

$$
f_{k_{t}, k_{t-1} \mid k_{t-2}^{*}, k_{t-3}^{*}, m_{t-1}, l_{t-1}}=\int f_{k_{t}, k_{t-1} \mid k_{t-2}^{*}, k_{t-3}^{*}, m_{t}, l_{t}, m_{t-1}, l_{t-1}} f_{m_{t}, l_{t} \mid k_{t-2}^{*}, k_{t-3}^{*}, m_{t-1}, l_{t-1}} d m_{t} d l_{t}
$$

because $f_{k_{t}, k_{t-1} \mid k_{t-2}^{*}, k_{t-3}^{*}, m_{t}, l_{t}, m_{t-1}, l_{t-1}}$ is identified from Theorem 4.2 and $f_{m_{t}, l_{t} \mid k_{t-2}^{*}, k_{t-3}^{*}, m_{t-1}, l_{t-1}}$ is observed from the data. Then from (14), $f_{l_{t} \mid k_{t}, k_{t-1}, m_{t-1}, l_{t-1}}$ is also identified because $f_{l_{t} \mid k_{t-2}^{*}, k_{t-3}^{*}, m_{t-1}, l_{t-1}}$ is observed from the data.

Similarly, we can identify $f_{m_{t} \mid k_{t}, k_{t-1}, m_{t-1}, l_{t-1}}$. We have from Assumption B.1.2 that,

$$
\begin{aligned}
f_{m_{t} \mid k_{t-2}^{*}, k_{t-3}^{*}, m_{t-1}, l_{t-1}} & =\int f_{m_{t} \mid k_{t}, k_{t-1}, k_{t-2}^{*}, k_{t-3}^{*}, m_{t-1}, l_{t-1}} f_{k_{t}, k_{t-1} \mid k_{t-2}^{*}, k_{t-3}^{*}, m_{t-1}, l_{t-1}} d k_{t} d k_{t-1} \\
& =\int f_{m_{t} \mid k_{t}, k_{t-1}, m_{t-1}, l_{t-1}} f_{k_{t}, k_{t-1} \mid k_{t-2}^{*}, k_{t-3}^{*}, m_{t-1}, l_{t-1}} d k_{t} d k_{t-1}
\end{aligned}
$$

which, in operator notation, is $L_{m_{t} \mid k_{t-2}^{*}, k_{t-3}^{*}, m_{t-1}, l_{t-1}}=L_{m_{t} \mid k_{t}, k_{t-1}, m_{t-1}, l_{t-1}} L_{k_{t}, k_{t-1} \mid k_{t-2}^{*}, k_{t-3}^{*}, m_{t-1}, l_{t-1}}$. Then, from Assumption B.1.3, we have that

$$
L_{m_{t} \mid k_{t}, k_{t-1}, m_{t-1}, l_{t-1}}=L_{m_{t} \mid k_{t-2}^{*}, k_{t-3}^{*}, m_{t-1}, l_{t-1}} L_{k_{t}, k_{t-1} \mid k_{t-2}^{*}, k_{t-3}^{*}, m_{t-1}, l_{t-1}}
$$

Since $f_{k_{t}, k_{t-1} \mid k_{t-2}^{*}, k_{t-3}^{*}, m_{t-1}, l_{t-1}}$ is already shown to be identified above and $f_{m_{t} \mid k_{t-2}^{*}, k_{t-3}^{*}, m_{t-1}, l_{t-1}}$ is observed from the data, $f_{m_{t} \mid k_{t}, k_{t-1}, m_{t-1}, l_{t-1}}$ is identified. We summarize the results.

Corollary B.2. Suppose Assumptions 4.1 and B.1 hold. Then we identify $f_{l_{t} \mid k_{t}, k_{t-1}, m_{t-1}, l_{t-1}}$ and $f_{m_{t} \mid k_{t}, k_{t-1}, m_{t-1}, l_{t-1}}$ from the observations $\left(y_{t}, k_{t}^{*}, k_{t-1}^{*}, k_{t-2}^{*}, k_{t-3}^{*}, m_{t}, l_{t}, m_{t-1}, l_{t-1}\right)$.

\section{Proof of Asymptotic Results}

Recall that $\tilde{\mathbf{x}}_{t}=\left(k_{t}, k_{t-1}, m_{t-1}, l_{t-1}\right), \mathbf{x}_{t}=\left(\tilde{\mathbf{x}}_{t}, l_{t}, m_{t}\right), \mathbf{s}_{t}=\left(m_{t}, l_{t}, m_{t-1}, l_{t-1}\right), \mathbf{z}_{t}=\left(k_{t}, k_{t-1}, \mathbf{s}_{t}\right)$ and $\mathbf{z}_{t}^{*}=\left(k_{t-2}^{*}, k_{t-3}^{*}, \mathbf{s}_{t}\right)$. Define 


$$
\begin{aligned}
Q(\theta) & =E\left[g\left(\mathbf{x}_{t} ; \theta\right)^{\prime} A\left(\mathbf{x}_{t}\right) g\left(\mathbf{x}_{t} ; \theta\right)\right] \\
& =\int g\left(\mathbf{x}_{t} ; \theta\right)^{\prime} A\left(\mathbf{x}_{t}\right) g\left(\mathbf{x}_{t} ; \theta\right) f_{k_{t}, k_{t-1} \mid \mathbf{s}_{t}} f_{\mathbf{s}_{t}} d x_{t} \\
Q_{n}(\theta) & =\frac{1}{n} \sum_{i=1}^{n} \int g\left(\mathbf{x}_{t i} ; \theta\right)^{\prime} A\left(\mathbf{x}_{t i}\right) g\left(\mathbf{x}_{t i} ; \theta\right) f_{k_{t}, k_{t-1} \mid \mathbf{s}_{t i}} d k_{t} d k_{t-1} \\
\widehat{Q}_{n}(\theta) & =\frac{1}{n} \sum_{i=1}^{n} \int \hat{g}\left(\mathbf{x}_{t i} ; \theta\right)^{\prime} \hat{A}\left(\mathbf{x}_{t i}\right) \hat{g}\left(\mathbf{x}_{t i} ; \theta\right) \hat{f}_{k_{t}, k_{t-1} \mid \mathbf{s}_{t i}} d k_{t} d k_{t-1} .
\end{aligned}
$$

\section{C.1 Consistency: Proof of Theorem 5.10}

The first result (Theorem 5.10.1) follows from a similar argument to the proof of Lemma 2 in Hu and Schennach (2008). In the proof we obtain the bound for the pathwise first derivative by

$$
\begin{aligned}
& \left|\frac{d}{d \tau} \ln f_{r_{t}, k_{t}^{*}, k_{t-1}^{*} \mid \mathbf{z}_{t}^{*}}\left(r_{t}, k_{t}^{*}, k_{t-1}^{*} \mid \mathbf{z}_{t}^{*} ; \bar{\alpha}+\tau\left(\alpha_{1}-\alpha_{2}\right)\right)\right|_{\tau=0} \\
\leq & \frac{1}{\left|f_{r_{t}, k_{t}^{*}, k_{t-1}^{*} \mid \mathbf{z}_{t}^{*}}\left(r_{t}, k_{t}^{*}, k_{t-1}^{*} \mid \mathbf{z}_{t}^{*} ; \bar{\alpha}\right)\right|} \\
& \times\left\{\int\left|\frac{d}{d \psi} f_{r_{t} \mid \mathbf{z}_{t}}\left(r_{t} \mid \mathbf{z}_{t} ; \bar{\psi}\right) \nu^{-1}(\cdot) \bar{f}_{1}\left(k_{t}^{*}, k_{t-1}^{*} \mid \mathbf{z}_{t}\right) \bar{f}_{2}\left(k_{t}, k_{t-1} \mid \mathbf{z}_{t}^{*}\right)\right| d k_{t} d k_{t-1}\right. \\
& +\int\left|f_{r_{t} \mid \mathbf{z}_{t}}\left(r_{t} \mid \mathbf{z}_{t} ; \bar{\psi}\right) \nu^{-1}\left(k_{t}^{*}, k_{t-1}^{*}, \mathbf{z}_{t}\right) \bar{f}_{2}\left(k_{t}, k_{t-1} \mid \mathbf{z}_{t}^{*}\right)\right| d k_{t} d k_{t-1} \\
& \left.+\int\left|f_{r_{t} \mid \mathbf{z}_{t}^{*}}\left(r_{t} \mid \mathbf{z}_{t} ; \bar{\psi}\right) \bar{f}_{1}\left(k_{t}^{*}, k_{t-1}^{*} \mid \mathbf{z}_{t}\right) \nu^{-1}\left(k_{t}, k_{t-1}, \mathbf{z}_{t}^{*}\right)\right| d k_{t} d k_{t-1}\right\}\left\|\alpha_{1}-\alpha_{2}\right\|_{s, \alpha} \\
\equiv & \left|\frac{f_{r_{t}, k_{t}^{*}, k_{t-1}^{*} \mid \mathbf{z}_{t}^{*}}^{|1|}\left(r_{t}, k_{t}^{*}, k_{t-1}^{*} \mid \mathbf{z}_{t}^{*} ; \bar{\alpha}, \nu\right)}{f_{r_{t}, k_{t}^{*}, k_{t-1}^{*} \mid \mathbf{z}_{t}^{*}}\left(r_{t}, k_{t}^{*}, k_{t-1}^{*} \mid \mathbf{z}_{t}^{*} ; \bar{\alpha}\right)}\right|\left\|\alpha_{1}-\alpha_{2}\right\|_{s, \alpha}
\end{aligned}
$$

where $\bar{\alpha}=\left(\bar{\psi}, \bar{f}_{1}, \bar{f}_{2}\right) \in \mathcal{A}$.

The second result (Theorem 5.10.2) can be proved by verifying the three conditions i)-iii) of Lemma A.1 of Newey and Powell (2003). Condition i) is explicitly assumed in Assumption 5.5. Condition iii) is satisfied by choosing $\Pi_{n} \theta \in \Theta_{n}$ such that $\left\|\Pi_{n} \theta-\theta\right\|_{s, \theta}=o(1)$ in Assumption 5.8.2.

We now verify Condition ii) of their Lemma A.1 by checking Conditions (i)-(iii) in Lemma A.2 of Newey and Powell (2003). First, Assumption 5.8.1 assumes their compactness of a parameter space in Lemma A.2 Condition (i). We verify their Lemma A.2 Condition (ii) about pointwise convergence in $\theta$. Note that for a generic positive constant $C$, 


$$
\begin{aligned}
& \left|\widehat{Q}_{n}(\theta)-Q_{n}(\theta)\right| \\
\leq & \frac{C}{n} \cdot\left|\sum_{i=1}^{n} \int\left(\left\|\hat{g}\left(\mathbf{x}_{t i} ; \theta\right) \hat{f}_{k_{t}, k_{t-1} \mid \mathbf{s}_{t i}}^{\frac{1}{2}}\right\|_{E}^{2}-\left\|g\left(\mathbf{x}_{t i} ; \theta\right) f_{k_{t}, k_{t-1} \mid \mathbf{s}_{t i}}^{\frac{1}{2}}\right\|_{E}^{2}\right) d k_{t} d k_{t-1}\right| \\
\leq & \frac{C}{n} \cdot \int\left(\sum_{i=1}^{n}\left\|\hat{g}\left(\mathbf{x}_{t i} ; \theta\right) \hat{f}_{k_{t}, k_{t-1} \mid \mathbf{s}_{t i}}^{\frac{1}{2}}-g\left(\mathbf{x}_{t i} ; \theta\right) f_{k_{t}, k_{t-1} \mid \mathbf{s}_{t i}}^{\frac{1}{2}}\right\|_{E}^{2}\right. \\
& \left.+2 \sum_{i=1}^{n}\left\|g\left(\mathbf{x}_{t i} ; \theta\right) f_{k_{t}, k_{t-1} \mid \mathbf{s}_{t i}}^{\frac{1}{2}}\right\|_{E} \sum_{i=1}^{n}\left\|\hat{g}\left(\mathbf{x}_{t i} ; \theta\right) \hat{f}_{k_{t}, k_{t-1} \mid \mathbf{s}_{t i}}^{\frac{1}{2}}-g\left(\mathbf{x}_{t i} ; \theta\right) f_{k_{t}, k_{t-1} \mid \mathbf{s}_{t i}}^{\frac{1}{2}}\right\|_{E}\right) d k_{t} d k_{t-1},
\end{aligned}
$$

by Assumption 5.6. Since $\sum_{i=1}^{n}\left\|g\left(\mathbf{x}_{t i} ; \theta\right) f_{k_{t}, k_{t-1} \mid \mathbf{s}_{t i}}^{\frac{1}{2}}\right\|_{E}^{2} / n=O_{p}(1)$ by the Markov inequality from Assumption 5.7.1, it suffices to show

$$
\sum_{i=1}^{n}\left\|\hat{g}\left(\mathbf{x}_{t i} ; \theta\right) \hat{f}_{k_{t}, k_{t-1} \mid \mathbf{s}_{t i}}^{\frac{1}{2}}-g\left(\mathbf{x}_{t i} ; \theta\right) f_{k_{t}, k_{t-1} \mid \mathbf{s}_{t i}}^{\frac{1}{2}}\right\|_{E}^{2} / n=o_{p}(1)
$$

to verify $\left|\widehat{Q}_{n}(\theta)-Q_{n}(\theta)\right|=o_{p}(1)$.

We note that for any given $\left(k_{t}, k_{t-1}\right)$

$$
\begin{aligned}
& E\left[\sum_{i=1}^{n}\left\|\hat{g}\left(\mathbf{x}_{t i} ; \theta\right) \hat{f}_{k_{t}, k_{t-1} \mid \mathbf{s}_{t i}}^{\frac{1}{2}}-g\left(\mathbf{x}_{t i} ; \theta\right) f_{k_{t}, k_{t-1} \mid \mathbf{s}_{t i}}^{\frac{1}{2}}\right\|_{E}^{2} / n\right] \\
= & E\left[\frac{1}{n} \sum_{i=1}^{n}\left\{\hat{g}_{1}\left(\mathbf{x}_{t i} ; \cdot\right) \hat{f}_{k_{t}, k_{t-1} \mid \mathbf{s}_{t i}}^{\frac{1}{2}}-g_{1}\left(\mathbf{x}_{t i} ; \cdot\right) f_{k_{t}, k_{t-1} \mid \mathbf{s}_{t i}}^{\frac{1}{2}}\right\}^{2}\right] \\
& +E\left[\frac{1}{n} \sum_{i=1}^{n}\left\{\hat{g}_{2}\left(\tilde{\mathbf{x}}_{t i} ; \cdot\right) \hat{f}_{k_{t}, k_{t-1} \mid \mathbf{s}_{t i}}^{\frac{1}{2}}-g_{2}\left(\tilde{\mathbf{x}}_{t i} ; \cdot\right) f_{k_{t}, k_{t-1} \mid \mathbf{s}_{t i}}^{\frac{1}{2}}\right\}^{2}\right] \\
= & E\left[\frac { 1 } { n } \sum _ { i = 1 } ^ { n } \left\{\left(\left(\int y_{t} \hat{f}_{y_{t} \mid k_{t}, k_{t-1}, \mathbf{s}_{t i}} d y_{t}-\beta_{l} l_{t i}-\beta_{k} k_{t}-\beta_{m} m_{t i}-\mathbf{c}\left(m_{t i}, k_{t}\right)^{\prime} \beta_{\omega}\right) \hat{f}_{k_{t}, k_{t-1} \mid \mathbf{s}_{t i}}^{\frac{1}{2}}\right.\right.\right. \\
& \left.-\left(\int y_{t} f_{y_{t} \mid k_{t}, k_{t-1}, \mathbf{s}_{t i}} d y_{t}-\beta_{l} l_{t i}-\beta_{k} k_{t}-\beta_{m} m_{t i}-\mathbf{c}\left(m_{t i}, k_{t}\right)^{\prime} \beta_{\omega}\right) f_{k_{t}, k_{t-1} \mid \mathbf{s}_{t i}}^{\frac{1}{2}}\right)^{2} \\
& +\left(\int y_{t} \hat{f}_{y_{t} \mid k_{t}, k_{t-1}, m_{t-1, i}, l_{t-1, i}} d y_{t}-\beta_{l} \int l_{t} \hat{f}_{l_{t} \mid k_{t}, k_{t-1}, m_{t-1, i}, l_{t-1, i}} d l_{t}-\beta_{k} k_{t}\right. \\
& \left.-\beta_{m} \int m_{t} \hat{f}_{m_{t} \mid k_{t}, k_{t-1}, m_{t-1, i}, l_{t-1, i}} d m_{t}-q\left(\mathbf{c}\left(m_{t-1, i}, k_{t-1}\right)^{\prime} \beta_{\omega}\right)\right) \hat{f}_{k_{t}, k_{t-1} \mid \mathbf{s}_{t i}}^{\frac{1}{2}} \\
& -\left(\int y_{t} f_{y_{t} \mid k_{t}, k_{t-1}, m_{t-1, i}, l_{t-1, i}} d y_{t}-\beta_{l} \int l_{t} f_{l_{t} \mid k_{t}, k_{t-1}, m_{t-1, i}, l_{t-1, i}} d l_{t}-\beta_{k} k_{t}\right. \\
& \left.\left.\left.\left.-\beta_{m} \int m_{t} f_{m_{t} \mid k_{t}, k_{t-1}, m_{t-1, i}, l_{t-1, i}} d m_{t}-q\left(\mathbf{c}\left(m_{t-1, i}, k_{t-1}\right)^{\prime} \beta_{\omega}\right)\right) f_{k_{t}, k_{t-1} \mid \mathbf{s}_{t i}}^{\frac{1}{2}}\right)^{2}\right\}\right]
\end{aligned}
$$




$$
\begin{aligned}
& =E\left[\frac { 1 } { n } \sum _ { i = 1 } ^ { n } \left\{\left(\int y_{t}\left(\hat{f}_{y_{t} \mid k_{t}, k_{t-1}, \mathbf{s}_{t i}} \hat{f}_{k_{t}, k_{t-1} \mid \mathbf{s}_{t i}}^{\frac{1}{2}}-f_{y_{t} \mid k_{t}, k_{t-1}, \mathbf{s}_{t i}} f_{k_{t}, k_{t-1} \mid \mathbf{s}_{t i}}^{\frac{1}{2}}\right) d y_{t}\right.\right.\right. \\
& \left.-\left(\beta_{l} l_{t i}+\beta_{k} k_{t}+\beta_{m} m_{t i}+\mathbf{c}\left(m_{t i}, k_{t}\right)^{\prime} \beta_{\omega}\right)\left(\hat{f}_{k_{t}, k_{t-1} \mid \mathbf{s}_{t i}}^{\frac{1}{2}}-f_{k_{t}, k_{t-1} \mid \mathbf{s}_{t i}}^{\frac{1}{2}}\right)\right)^{2} \\
& +\left(\int y_{t}\left(\hat{f}_{y_{t} \mid k_{t}, k_{t-1}, m_{t-1, i}, l_{t-1, i}} \hat{f}_{k_{t}, k_{t-1} \mid \mathbf{s}_{t i}}^{\frac{1}{2}}-f_{y_{t} \mid k_{t}, k_{t-1}, \mathbf{s}_{t i}} f_{k_{t}, k_{t-1} \mid \mathbf{s}_{t i}}^{\frac{1}{2}}\right) d y_{t}\right. \\
& -\beta_{l} \int l_{t}\left(\hat{f}_{l_{t} \mid k_{t}, k_{t-1}, m_{t-1, i}, l_{t-1, i}} \hat{f}_{k_{t}, k_{t-1} \mid \mathbf{s}_{t i}}^{\frac{1}{2}}-f_{y_{t} \mid k_{t}, k_{t-1}, \mathbf{s}_{t i}} f_{k_{t}, k_{t-1} \mid \mathbf{s}_{t i}}^{\frac{1}{2}}\right) d l_{t} \\
& -\beta_{m} \int m_{t}\left(\hat{f}_{m_{t} \mid k_{t}, k_{t-1}, m_{t-1, i}, l_{t-1, i}} \hat{f}_{k_{t}, k_{t-1} \mid \mathbf{s}_{t i}}^{\frac{1}{2}}-f_{m_{t} \mid k_{t}, k_{t-1}, \mathbf{s}_{t i}} f_{k_{t}, k_{t-1} \mid \mathbf{s}_{t i}}^{\frac{1}{2}}\right) d m_{t} \\
& \left.\left.\left.-\left(\beta_{k} k_{t}+q\left(\mathbf{c}\left(m_{t-1, i}, k_{t-1}\right)^{\prime} \beta_{\omega}\right)\right)\left(\hat{f}_{k_{t}, k_{t-1} \mid \mathbf{s}_{t i}}^{\frac{1}{2}}-f_{k_{t}, k_{t-1} \mid \mathbf{s}_{t i}}^{\frac{1}{2}}\right)\right)^{2}\right\}\right] \\
& =E\left[\frac { 1 } { n } \sum _ { i = 1 } ^ { n } \left\{\left(\int y_{t} \hat{f}_{y_{t} \mid k_{t}, k_{t-1}, \mathbf{s}_{t i}} d y_{t}\left(\hat{f}_{k_{t}, k_{t-1} \mid \mathbf{s}_{t i}}^{\frac{1}{2}}-f_{k_{t}, k_{t-1} \mid \mathbf{s}_{t i}}^{\frac{1}{2}}\right)\right.\right.\right. \\
& +\int y_{t}\left(\hat{f}_{y_{t} \mid k_{t}, k_{t-1}, \mathbf{s}_{t i}}-f_{y_{t} \mid k_{t}, k_{t-1}, \mathbf{s}_{t i}}\right) d y_{t} f_{k_{t}, k_{t-1} \mid \mathbf{s}_{t i}}^{\frac{1}{2}} \\
& \left.-\left(\beta_{l} l_{t i}+\beta_{k} k_{t}+\beta_{m} m_{t i}+\mathbf{c}\left(m_{t i}, k_{t}\right)^{\prime} \beta_{\omega}\right)\left(\hat{f}_{k_{t}, k_{t-1} \mid \mathbf{s}_{t i}}^{\frac{1}{2}}-f_{k_{t}, k_{t-1} \mid \mathbf{s}_{t i}}^{\frac{1}{2}}\right)\right)^{2} \\
& +\left(\int y_{t} \hat{f}_{y_{t} \mid k_{t}, k_{t-1}, m_{t-1, i}, l_{t-1, i}} d y_{t}\left(\hat{f}_{k_{t}, k_{t-1} \mid \mathbf{s}_{t i}}^{\frac{1}{2}}-f_{k_{t}, k_{t-1} \mid \mathbf{s}_{t i}}^{\frac{1}{2}}\right)\right. \\
& +\left(\int y_{t}\left(\hat{f}_{y_{t} \mid k_{t}, k_{t-1}, m_{t-1, i}, l_{t-1, i}}-f_{y_{t} \mid k_{t}, k_{t-1}, \mathbf{s}_{t i}}\right) d y_{t} f_{k_{t}, k_{t-1} \mid \mathbf{s}_{t i}}^{\frac{1}{2}}\right. \\
& -\beta_{l}\left(\int l_{t} \hat{f}_{l_{t} \mid k_{t}, k_{t-1}, m_{t-1, i}, l_{t-1, i}} d l_{t}\left(\hat{f}_{k_{t}, k_{t-1} \mid \mathbf{s}_{t i}}^{\frac{1}{2}}-f_{k_{t}, k_{t-1} \mid \mathbf{s}_{t i}}^{\frac{1}{2}}\right)\right. \\
& \left.+\int l_{t}\left(\hat{f}_{l_{t} \mid k_{t}, k_{t-1}, m_{t-1, i}, l_{t-1, i}}-f_{l_{t} \mid k_{t}, k_{t-1}, \mathbf{s}_{t i}}\right) d l_{t} f_{k_{t}, k_{t-1} \mid \mathbf{s}_{t i}}^{\frac{1}{2}}\right) \\
& -\beta_{m}\left(\int m_{t} \hat{f}_{m_{t} \mid k_{t}, k_{t-1}, m_{t-1, i}, l_{t-1, i}} d m_{t}\left(\hat{f}_{k_{t}, k_{t-1} \mid \mathbf{s}_{t i}}^{\frac{1}{2}}-f_{k_{t}, k_{t-1} \mid \mathbf{s}_{t i}}^{\frac{1}{2}}\right)\right. \\
& \left.+\int m_{t}\left(\hat{f}_{m_{t} \mid k_{t}, k_{t-1}, m_{t-1, i}, l_{t-1, i}}-f_{m_{t} \mid k_{t}, k_{t-1}, \mathbf{s}_{t i}}\right) d m_{t} f_{k_{t}, k_{t-1} \mid \mathbf{s}_{t i}}^{\frac{1}{2}}\right) \\
& \left.\left.\left.-\left(\beta_{k} k_{t}+q\left(\mathbf{c}\left(m_{t-1, i}, k_{t-1}\right)^{\prime} \beta_{\omega}\right)\right)\left(\hat{f}_{k_{t}, k_{t-1} \mid \mathbf{s}_{t i}}^{\frac{1}{2}}-f_{k_{t}, k_{t-1} \mid \mathbf{s}_{t i}}^{\frac{1}{2}}\right)\right)^{2}\right\}\right]
\end{aligned}
$$




$$
\begin{aligned}
& \leq E\left[\frac { 1 } { n } \sum _ { i = 1 } ^ { n } \left\{\left(\int\left|y_{t} \hat{f}_{y_{t} \mid k_{t}, k_{t-1}, \mathbf{s}_{t i}}\right| d y_{t}\left|\nu^{-1}\left(k_{t}, k_{t-1}, \mathbf{z}_{t}^{*}\right)\right|\left\|\hat{f}_{2}^{\frac{1}{2}}-f_{2}^{\frac{1}{2}}\right\|_{\infty, v}\right.\right.\right. \\
& +\int\left|y_{t} \frac{d f_{y_{t} \mid k_{t}, k_{t-1}, \mathbf{s}_{t i} ; \psi}}{d \psi} \nu^{-1}(\cdot)\right|\|\hat{\psi}-\psi\|_{\infty, v} d y_{t}\left|f_{k_{t}, k_{t-1} \mid \mathbf{s}_{t i}}^{\frac{1}{2}}\right| \\
& \left.+\left|\beta_{l} l_{t i}+\beta_{k} k_{t}+\beta_{m} m_{t i}+\mathbf{c}\left(m_{t i}, k_{t}\right)^{\prime} \beta_{\omega}\right|\left|\nu^{-1}\left(k_{t}, k_{t-1}, \mathbf{z}_{t}^{*}\right)\right|\left\|\hat{f}_{2}^{\frac{1}{2}}-f_{2}^{\frac{1}{2}}\right\|_{\infty, v}\right)^{2} \\
& +\left(\left|\int y_{t} \hat{f}_{y_{t} \mid k_{t}, k_{t-1}, m_{t-1, i}, l_{t-1, i}} d y_{t}\right|\left|\nu^{-1}\left(k_{t}, k_{t-1}, \mathbf{z}_{t}^{*}\right)\right|\left\|\hat{f}_{2}^{\frac{1}{2}}-f_{2}^{\frac{1}{2}}\right\|_{\infty, v}\right. \\
& +\int\left|y_{t} \frac{d f_{y_{t} \mid k_{t}, k_{t-1}, m_{t-1, i}, l_{t-1, i} ; \psi}}{d \psi} \nu^{-1}(\cdot)\right|\|\hat{\psi}-\psi\|_{\infty, v} d y_{t}\left|f_{k_{t}, k_{t-1} \mid \mathbf{s}_{t i}}^{\frac{1}{2}}\right| \\
& +\left|\beta_{l} \int l_{t} \hat{f}_{l_{t} \mid k_{t}, k_{t-1}, m_{t-1}, l_{t-1}} d l_{t}\right|\left|\nu^{-1}\left(k_{t}, k_{t-1}, \mathbf{z}_{t}^{*}\right)\right|\left\|\hat{f}_{2}^{\frac{1}{2}}-f_{2}^{\frac{1}{2}}\right\|_{\infty, v} \\
& +\left|\beta_{l}\right| \int\left|l_{t} \frac{d f_{l_{t} \mid k_{t}, k_{t-1}, m_{t-1, i}, l_{t-1, i} ; \psi}}{d \psi} \nu^{-1}(\cdot)\right|\|\hat{\psi}-\psi\|_{\infty, v} d l_{t}\left|f_{k_{t}, k_{t-1} \mid \mathbf{s}_{t i}}^{\frac{1}{2}}\right| \\
& +\left|\beta_{m} \int m_{t} \hat{f}_{m_{t} \mid k_{t}, k_{t-1}, m_{t-1, i}, l_{t-1, i}} d m_{t}\right|\left|\nu^{-1}\left(k_{t}, k_{t-1}, \mathbf{z}_{t}^{*}\right)\right|\left\|\hat{f}_{2}^{\frac{1}{2}}-f_{2}^{\frac{1}{2}}\right\|_{\infty, v} \\
& +\left|\beta_{m}\right| \int\left|m_{t} \frac{d f_{m_{t} \mid k_{t}, k_{t-1}, m_{t-1, i}, l_{t-1, i} ; \psi}}{d \psi} \nu^{-1}(\cdot)\right|\|\hat{\psi}-\psi\|_{\infty, v} d m_{t}\left|f_{k_{t}, k_{t-1} \mid \mathbf{s}_{t i}}^{\frac{1}{2}}\right| \\
& \left.\left.\left.+\left|\beta_{k} k_{t}+q\left(\mathbf{c}\left(m_{t-1, i}, k_{t-1}\right)^{\prime} \beta_{\omega}\right)\right|\left|\nu^{-1}\left(k_{t}, k_{t-1}, \mathbf{z}_{t}^{*}\right)\right|\left\|\hat{f}_{2}^{\frac{1}{2}}-f_{2}^{\frac{1}{2}}\right\|_{\infty, v}\right)^{2}\right\}\right] \\
& \leq B_{1 n} E\|\hat{\alpha}-\alpha\|_{s, \alpha}^{2}=o(1) \text {, }
\end{aligned}
$$

because

$$
\begin{aligned}
& B_{1 n} \\
& \equiv\left[\frac { 1 } { n } \sum _ { i = 1 } ^ { n } E \left\{\left(\int\left|y_{t} \hat{f}_{y_{t} \mid k_{t}, k_{t-1}, \mathbf{s}_{t i}}\right| d y_{t}\left|\nu^{-1}\left(k_{t}, k_{t-1}, \mathbf{z}_{t}^{*}\right)\right|+\int\left|y_{t} \frac{d f_{y_{t}} \mid k_{t}, k_{t-1}, \mathbf{s}_{t i} ; \psi}{d \psi} \nu^{-1}(\cdot)\right| d y_{t}\left|f_{k_{t}, k_{t-1} \mid \mathbf{s}_{t i}}^{\frac{1}{2}}\right|\right.\right.\right. \\
& \left.+\left|\beta_{l} l_{t i}+\beta_{k} k_{t}+\beta_{m} m_{t i}+\mathbf{c}\left(m_{t i}, k_{t}\right)^{\prime} \beta_{\omega}\right|\left|\nu^{-1}\left(k_{t}, k_{t-1}, \mathbf{z}_{t}^{*}\right)\right|\right)^{2} \\
& +\left(\left|\int y_{t} \hat{f}_{y_{t} \mid k_{t}, k_{t-1}, m_{t-1, i}, l_{t-1, i}} d y_{t}\right|\left|\nu^{-1}\left(k_{t}, k_{t-1}, \mathbf{z}_{t}^{*}\right)\right|+\int\left|y_{t} \frac{d f_{y_{t} \mid k_{t}, k_{t-1}, m_{t-1, i}, l_{t-1, i} ; \psi}}{d \psi} \nu^{-1}(\cdot)\right| d y_{t}\left|f_{k_{t}, k_{t-1} \mid \mathbf{s}_{t i}}^{\frac{1}{2}}\right|\right. \\
& +\left|\beta_{l} \int l_{t} \hat{f}_{l_{t} \mid k_{t}, k_{t-1}, m_{t-1, i}, l_{t-1, i}} d l_{t}\right|\left|\nu^{-1}\left(k_{t}, k_{t-1}, \mathbf{z}_{t}^{*}\right)\right|+\left|\beta_{l}\right| \int\left|l_{t} \frac{d f_{l_{t} \mid k_{t}, k_{t-1}, m_{t-1, i}, l_{t-1, i} ;}}{d \psi} \nu^{-1}(\cdot)\right| d l_{t}\left|f_{k_{t}, k_{t-1} \mid \mathbf{s}_{t i}}^{\frac{1}{2}}\right| \\
& +\left|\beta_{m} \int m_{t} \hat{f}_{m_{t} \mid k_{t}, k_{t-1}, m_{t-1, i}, l_{t-1, i}} d m_{t}\right|\left|\nu^{-1}\left(k_{t}, k_{t-1}, \mathbf{z}_{t}^{*}\right)\right| \\
& +\left|\beta_{m}\right| \int\left|m_{t} \frac{d f_{m_{t} \mid k_{t}, k_{t-1}, m_{t-1, i}, l_{t-1, i} ; \psi}}{d \psi} \nu^{-1}(\cdot)\right| d m_{t}\left|f_{k_{t}, k_{t-1} \mid \mathbf{s}_{t i}}^{\frac{1}{2}}\right| \\
& \left.\left.\left.+\left|\beta_{k} k_{t}+q\left(\mathbf{c}\left(m_{t-1, i}, k_{t-1}\right)^{\prime} \beta_{\omega}\right)\right|\left|\nu^{-1}\left(k_{t}, k_{t-1}, \mathbf{z}_{t}^{*}\right)\right|\right)^{2}\right\}\right]=O(1)
\end{aligned}
$$


and because $\|\hat{\alpha}-\alpha\|_{s, \alpha}^{2}=o_{p}(1)$.

We therefore obtain $\sum_{i=1}^{n}\left\|\hat{g}\left(\mathbf{x}_{t i} ; \theta\right) \hat{f}_{k_{t}, k_{t-1} \mid \mathbf{s}_{t i}}^{\frac{1}{2}}-g\left(\mathbf{x}_{t i} ; \theta\right) f_{k_{t}, k_{t-1} \mid \mathbf{s}_{t i}}^{\frac{1}{2}}\right\|_{E}^{2} / n=o_{p}(1)$ by the Markov inequality. Note that $Q_{n}(\theta)=Q(\theta)+o_{p}(1)$ by the weak law of large numbers. Therefore, we have verified Lemma A.2 Condition (ii) of Newey and Powell (2003) by the triangle inequality.

Next we verify Condition (iii) of their Lemma A.2. Since $\|\cdot\|_{s, \theta}^{\nu}$ is bounded on $\Theta \times \Theta$ by the compactness of the parameter space, there are constants $C, \tilde{C}$ such that

$$
\begin{aligned}
& \left|\widehat{Q}_{n}(\tilde{\theta})-\widehat{Q}_{n}(\theta)\right| \\
& \leq \frac{C}{n} \cdot\left|\sum_{i=1}^{n} \int\left(\left\|\hat{g}\left(\mathbf{x}_{t i} ; \tilde{\theta}\right) \hat{f}_{k_{t}, k_{t-1} \mid \mathbf{s}_{t i}}^{\frac{1}{2}}\right\|_{E}^{2}-\left\|\hat{g}\left(\mathbf{x}_{t i} ; \theta\right) \hat{f}_{k_{t}, k_{t-1} \mid \mathbf{s}_{t i}}^{\frac{1}{2}}\right\|_{E}^{2}\right) d k_{t} d k_{t-1}\right| \\
& \leq \frac{C}{n} \cdot\left(\sum_{i=1}^{n} \int\left(\left\|\hat{g}\left(\mathbf{x}_{t i} ; \tilde{\theta}\right) \hat{f}_{k_{t}, k_{t-1} \mid \mathbf{s}_{t i}}^{\frac{1}{2}}-\hat{g}\left(\mathbf{x}_{t i} ; \theta\right) \hat{f}_{k_{t}, k_{t-1} \mid \mathbf{s}_{t i}}^{\frac{1}{2}}\right\|_{E}^{2}\right) d k_{t} d k_{t-1}\right. \\
& +2 \sum_{i=1}^{n} \int\left\|\hat{g}\left(\mathbf{x}_{t i} ; \theta\right) \hat{f}_{k_{t}, k_{t-1} \mid \mathbf{s}_{t i}}^{\frac{1}{2}}\right\|_{E} d k_{t} d k_{t-1} \\
& \left.\times \sum_{i=1}^{n} \int\left(\left\|\hat{g}\left(\mathbf{x}_{t i} ; \tilde{\theta}\right) \hat{f}_{k_{t}, k_{t-1} \mid \mathbf{s}_{t i}}^{\frac{1}{2}}-\hat{g}\left(\mathbf{x}_{t i} ; \theta\right) \hat{f}_{k_{t}, k_{t-1} \mid \mathbf{s}_{t i}}^{\frac{1}{2}}\right\|_{E}\right) d k_{t} d k_{t-1}\right) \\
& =\frac{C}{n} \cdot\left(\sum_{i=1}^{n} \int\left(\left\|\hat{g}\left(\mathbf{x}_{t i} ; \tilde{\theta}\right) \hat{f}_{k_{t}, k_{t-1} \mid \mathbf{s}_{t i}}^{\frac{1}{2}}-\hat{g}\left(\mathbf{x}_{t i} ; \theta\right) \hat{f}_{k_{t}, k_{t-1} \mid \mathbf{s}_{t i}}^{\frac{1}{2}}\right\|_{E}^{2}\right) d k_{t} d k_{t-1}\right. \\
& +2 \sum_{i=1}^{n} \int\left(\left\|\hat{g}\left(\mathbf{x}_{t i} ; \theta\right) \hat{f}_{k_{t}, k_{t-1} \mid \mathbf{s}_{t i}}^{\frac{1}{2}}-\hat{g}\left(\mathbf{x}_{t i} ; \theta_{0}\right) \hat{f}_{k_{t}, k_{t-1} \mid \mathbf{s}_{t i}}^{\frac{1}{2}}\right\|_{E}\right) d k_{t} d k_{t-1} \\
& \times \sum_{i=1}^{n} \int\left(\left\|\hat{g}\left(\mathbf{x}_{t i} ; \tilde{\theta}\right) \hat{f}_{k_{t}, k_{t-1} \mid \mathbf{s}_{t i}}^{\frac{1}{2}}-\hat{g}\left(\mathbf{x}_{t i} ; \theta\right) \hat{f}_{k_{t}, k_{t-1} \mid \mathbf{s}_{t i}}^{\frac{1}{2}}\right\|_{E}\right) d k_{t} d k_{t-1} \\
& +2 \sum_{i=1}^{n} \int\left\|\hat{g}\left(\mathbf{x}_{t i} ; \theta_{0}\right) \hat{f}_{k_{t}, k_{t-1} \mid \mathbf{s}_{t i}}^{\frac{1}{2}}\right\|_{E} d k_{t} d k_{t-1} \\
& \left.\times \sum_{i=1}^{n} \int\left(\left\|\hat{g}\left(\mathbf{x}_{t i} ; \tilde{\theta}\right) \hat{f}_{k_{t}, k_{t-1} \mid \mathbf{s}_{t i}}^{\frac{1}{2}}-\hat{g}\left(\mathbf{x}_{t i} ; \theta\right) \hat{f}_{k_{t}, k_{t-1} \mid \mathbf{s}_{t i}}^{\frac{1}{2}}\right\|_{E}\right) d k_{t} d k_{t-1}\right)
\end{aligned}
$$




$$
\begin{aligned}
& =\frac{C}{n}\left\{\int \sum _ { i = 1 } ^ { n } \left[\left(\hat{g}_{1}\left(\mathbf{x}_{t i} ; \tilde{\beta}\right) \hat{f}_{k_{t}, k_{t-1} \mid \mathbf{s}_{t i}}^{\frac{1}{2}}-\hat{g}_{1}\left(\mathbf{x}_{t i} ; \beta\right) \hat{f}_{k_{t}, k_{t-1} \mid \mathbf{s}_{t i}}^{\frac{1}{2}}\right)^{2}\right.\right. \\
& \left.+\left(\hat{g}_{2}\left(\tilde{\mathbf{x}}_{t i} ; \tilde{\theta}\right) \hat{f}_{k_{t}, k_{t-1} \mid \mathbf{s}_{t i}}^{\frac{1}{2}}-g_{2}\left(\tilde{\mathbf{x}}_{t i} ; \theta\right) \hat{f}_{k_{t}, k_{t-1} \mid \mathbf{s}_{t i}}^{\frac{1}{2}}\right)^{2}\right] d k_{t} d k_{t-1} \\
& +2 \int\left\{\sum _ { i = 1 } ^ { n } \left[\left(\hat{g}_{1}\left(\mathbf{x}_{t i} ; \beta\right) \hat{f}_{k_{t}, k_{t-1} \mid \mathbf{s}_{t i}}^{\frac{1}{2}}-\hat{g}_{1}\left(\mathbf{x}_{t i} ; \beta_{0}\right) \hat{f}_{k_{t}, k_{t-1} \mid \mathbf{s}_{t i}}^{\frac{1}{2}}\right)^{2}\right.\right. \\
& \left.\left.+\left(\hat{g}_{2}\left(\tilde{\mathbf{x}}_{t i} ; \theta\right) \hat{f}_{k_{t}, k_{t-1} \mid \mathbf{s}_{t i}}^{\frac{1}{2}}-g_{2}\left(\tilde{\mathbf{x}}_{t i} ; \theta_{0}\right) \hat{f}_{k_{t}, k_{t-1} \mid \mathbf{s}_{t i}}^{\frac{1}{2}}\right)^{2}\right]\right\}^{1 / 2} d k_{t} d k_{t-1} \\
& \cdot \int\left\{\sum _ { i = 1 } ^ { n } \left[\left(\hat{g}_{1}\left(\mathbf{x}_{t i} ; \tilde{\beta}\right) \hat{f}_{k_{t}, k_{t-1} \mid \mathbf{s}_{t i}}^{\frac{1}{2}}-\hat{g}_{1}\left(\mathbf{x}_{t i} ; \beta\right) \hat{f}_{k_{t}, k_{t-1} \mid \mathbf{s}_{t i}}^{\frac{1}{2}}\right)^{2}\right.\right. \\
& \left.\left.+\left(\hat{g}_{2}\left(\tilde{\mathbf{x}}_{t i} ; \tilde{\theta}\right) \hat{f}_{k_{t}, k_{t-1} \mid \mathbf{s}_{t i}}^{\frac{1}{2}}-g_{2}\left(\tilde{\mathbf{x}}_{t i} ; \theta\right) \hat{f}_{k_{t}, k_{t-1} \mid \mathbf{s}_{t i}}^{\frac{1}{2}}\right)^{2}\right]\right\}^{1 / 2} d k_{t} d k_{t-1} \\
& +2 \int\left\{\sum_{i=1}^{n}\left(\hat{g}_{1}\left(\mathbf{x}_{t i} ; \beta_{0}\right)^{2} \hat{f}_{k_{t}, k_{t-1} \mid \mathbf{s}_{t i}}+\hat{g}_{2}\left(\tilde{\mathbf{x}}_{t i} ; \theta_{0}\right)^{2} \hat{f}_{k_{t}, k_{t-1} \mid \mathbf{s}_{t i}}\right)\right\}^{1 / 2} d k_{t} d k_{t-1} \\
& \cdot \int \sum_{i=1}^{n}\left[\left(\hat{g}_{1}\left(\mathbf{x}_{t i} ; \tilde{\beta}\right) \hat{f}_{k_{t}, k_{t-1} \mid \mathbf{s}_{t i}}^{\frac{1}{2}}-\hat{g}_{1}\left(\mathbf{x}_{t i} ; \beta\right) \hat{f}_{k_{t}, k_{t-1} \mid \mathbf{s}_{t i}}^{\frac{1}{2}}\right)^{2}\right. \\
& \left.\left.\left.+\left(\hat{g}_{2}\left(\tilde{\mathbf{x}}_{t i} ; \tilde{\theta}\right) \hat{f}_{k_{t}, k_{t-1} \mid \mathbf{s}_{t i}}^{\frac{1}{2}}-g_{2}\left(\tilde{\mathbf{x}}_{t i} ; \theta\right) \hat{f}_{k_{t}, k_{t-1} \mid \mathbf{s}_{t i}}^{\frac{1}{2}}\right)^{2}\right]\right\}^{1 / 2} d k_{t} d k_{t-1}\right\} \\
& =\frac{C}{n}\left\{\int \sum _ { i = 1 } ^ { n } \left[\left(\int\left(\rho_{1}\left(\mathbf{y}_{t} ; \tilde{\beta}\right)-\rho_{1}\left(\mathbf{y}_{t} ; \beta\right)\right) \hat{f}_{\mathbf{y}_{t} \mid \mathbf{x}_{t i}} d \mathbf{y}_{t} \hat{f}_{k_{t}, k_{t-1} \mid \mathbf{s}_{t i}}^{\frac{1}{2}}\right)^{2}\right.\right. \\
& \left.+\left(\int\left(\rho_{2}\left(\mathbf{y}_{t, t-1} ; \tilde{\theta}\right)-\rho_{2}\left(\mathbf{y}_{t, t-1} ; \theta\right)\right) \hat{f}_{\mathbf{y}_{t, t-1} \mid \tilde{\mathbf{x}}_{t i}} d \mathbf{y}_{t, t-1} \hat{f}_{k_{t}, k_{t-1} \mid \mathbf{s}_{t i}}^{\frac{1}{2}}\right)^{2}\right] d k_{t} d k_{t-1} \\
& +2 \int\left\{\sum _ { i = 1 } ^ { n } \left[\left(\int\left(\rho_{1}\left(\mathbf{y}_{t} ; \beta\right)-\rho_{1}\left(\mathbf{y}_{t} ; \beta_{0}\right)\right) \hat{f}_{\mathbf{y}_{t} \mid \mathbf{x}_{t i}} d \mathbf{y}_{t} \hat{f}_{k_{t}, k_{t-1} \mid \mathbf{s}_{t i}}^{\frac{1}{2}}\right)^{2}\right.\right. \\
& \left.\left.+\left(\int\left(\rho_{2}\left(\mathbf{y}_{t, t-1} ; \theta\right)-\rho_{2}\left(\mathbf{y}_{t, t-1} ; \theta_{0}\right)\right) \hat{f}_{\mathbf{y}_{t, t-1} \mid \tilde{\mathbf{x}}_{t i}} d \mathbf{y}_{t, t-1} \hat{f}_{k_{t}, k_{t-1} \mid \mathbf{s}_{t i}}^{\frac{1}{2}}\right)^{2}\right]\right\}^{1 / 2} d k_{t} d k_{t-1} \\
& \cdot \int \sum_{i=1}^{n}\left[\left(\int\left(\rho_{1}\left(\mathbf{y}_{t} ; \tilde{\beta}\right)-\rho_{1}\left(\mathbf{y}_{t} ; \beta\right)\right) \hat{f}_{\mathbf{y}_{t} \mid \mathbf{x}_{t i}} d \mathbf{y}_{t} \hat{f}_{k_{t}, k_{t-1} \mid \mathbf{s}_{t i}}^{\frac{1}{2}}\right)^{2}\right. \\
& \left.\left.+\left(\int\left(\rho_{2}\left(\mathbf{y}_{t, t-1} ; \tilde{\theta}\right)-\rho_{2}\left(\mathbf{y}_{t, t-1} ; \theta\right)\right) \hat{f}_{\mathbf{y}_{t, t-1} \mid \tilde{\mathbf{x}}_{t i}} d \mathbf{y}_{t, t-1} \hat{f}_{k_{t}, k_{t-1} \mid \mathbf{s}_{t i}}^{\frac{1}{2}}\right)^{2}\right]\right\}^{1 / 2} d k_{t} d k_{t-1}
\end{aligned}
$$




$$
\begin{aligned}
& +2 \int\left\{\sum _ { i = 1 } ^ { n } \left[\left(\int\left(\rho_{1}\left(\mathbf{y}_{t} ; \beta_{0}\right)\right) \hat{f}_{\mathbf{y}_{t} \mid \mathbf{x}_{t i}} d \mathbf{y}_{t}\right)^{2} \hat{f}_{k_{t}, k_{t-1} \mid \mathbf{s}_{t i}}\right.\right. \\
& \left.\left.+\left(\int \rho_{2}\left(\mathbf{y}_{t, t-1} ; \theta_{0}\right) \hat{f}_{\mathbf{y}_{t, t-1} \mid \tilde{\mathbf{x}}_{t i}} d \mathbf{y}_{t, t-1}\right)^{2} \hat{f}_{k_{t}, k_{t-1} \mid \mathbf{s}_{t i}}\right]\right\}^{1 / 2} d k_{t} d k_{t-1} \\
& \cdot \int\left\{\sum _ { i = 1 } ^ { n } \left[\left(\int\left(\rho_{1}\left(\mathbf{y}_{t} ; \tilde{\beta}\right)-\rho_{1}\left(\mathbf{y}_{t} ; \beta\right)\right) \hat{f}_{\mathbf{y}_{t} \mid \mathbf{x}_{t i}} d \mathbf{y}_{t} \hat{f}_{k_{t}, k_{t-1} \mid \mathbf{s}_{t i}}^{\frac{1}{2}}\right)^{2}\right.\right. \\
& \left.\left.\left.+\left(\int\left(\rho_{2}\left(\mathbf{y}_{t, t-1} ; \tilde{\theta}\right)-\rho_{2}\left(\mathbf{y}_{t, t-1} ; \theta\right)\right) \hat{f}_{\mathbf{y}_{t, t-1} \mid \tilde{\mathbf{x}}_{t i}} d \mathbf{y}_{t, t-1} \hat{f}_{k_{t}, k_{t-1} \mid \mathbf{s}_{t i}}^{\frac{1}{2}}\right)^{2}\right]\right\}^{1 / 2} d k_{t} d k_{t-1}\right\} \\
& \leq \frac{C}{n}\left\{\int \sum_{i=1}^{n}\left(\int b_{1}\left(\mathbf{y}_{t}\right) \hat{f}_{\mathbf{y}_{t} \mid \mathbf{x}_{t i}} d \mathbf{y}_{t} \hat{f}_{k_{t}, k_{t-1} \mid \mathbf{s}_{t i}}^{\frac{1}{2}}\right)^{2} \cdot\|\tilde{\theta}-\theta\|_{s, \theta}^{2 \nu} d k_{t} d k_{t-1}\right. \\
& +\int \sum_{i=1}^{n}\left(\int b_{2}\left(\mathbf{y}_{t, t-1}\right) \hat{f}_{\mathbf{y}_{t, t-1} \mid \tilde{\mathbf{x}}_{t i}} d \mathbf{y}_{t, t-1} \hat{f}_{k_{t}, k_{t-1} \mid \mathbf{s}_{t i}}^{\frac{1}{2}}\right)^{2} \cdot\|\tilde{\theta}-\theta\|_{s, \theta}^{2 \nu} d k_{t} d k_{t-1} \\
& +2 \int\left\{\sum_{i=1}^{n}\left[\left(\int b_{1}\left(\mathbf{y}_{t}\right) \hat{f}_{\mathbf{y}_{t} \mid \mathbf{x}_{t i}} d \mathbf{y}_{t} \hat{f}_{k_{t}, k_{t-1} \mid \mathbf{s}_{t i}}^{\frac{1}{2}}\right)^{2}+\left(\int b_{2}\left(\mathbf{y}_{t, t-1}\right) \hat{f}_{\mathbf{y}_{t, t-1} \mid \tilde{\mathbf{x}}_{t i}} d \mathbf{y}_{t, t-1} \hat{f}_{k_{t}, k_{t-1} \mid \mathbf{s}_{t i}}^{\frac{1}{2}}\right)^{2}\right]^{1 / 2}\right. \\
& \cdot\left\|\theta-\theta_{0}\right\|_{s, \theta}^{\nu} d k_{t} d k_{t-1} \\
& \cdot \int\left\{\sum_{i=1}^{n}\left[\left(\int b_{1}\left(\mathbf{y}_{t}\right) \hat{f}_{\mathbf{y}_{t} \mid \mathbf{x}_{t i}} d \mathbf{y}_{t} \hat{f}_{k_{t}, k_{t-1} \mid \mathbf{s}_{t i}}^{\frac{1}{2}}\right)^{2}+\left(\int b_{2}\left(\mathbf{y}_{t, t-1}\right) \hat{f}_{\mathbf{y}_{t, t-1} \mid \tilde{\mathbf{x}}_{t i}} d \mathbf{y}_{t, t-1} \hat{f}_{k_{t}, k_{t-1} \mid \mathbf{s}_{t i}}^{\frac{1}{2}}\right)^{2}\right]^{1 / 2}\right. \\
& \cdot\|\tilde{\theta}-\theta\|_{s, \theta}^{\nu} d k_{t} d k_{t-1} \\
& +2 \int\left\{\sum _ { i = 1 } ^ { n } \left[\left(\int\left(\rho_{1}\left(\mathbf{y}_{t} ; \beta_{0}\right)\right) \hat{f}_{\mathbf{y}_{t} \mid \mathbf{x}_{t i}} d \mathbf{y}_{t}\right)^{2}\right.\right. \\
& \left.\left.+\left(\int \rho_{2}\left(\mathbf{y}_{t, t-1} ; \theta_{0}\right) \hat{f}_{\mathbf{y}_{t, t-1} \mid \tilde{\mathbf{x}}_{t i}} d \mathbf{y}_{t, t-1}\right)^{2}\right]\right\}^{1 / 2} \hat{f}_{k_{t}, k_{t-1} \mid \mathbf{s}_{t i}}^{\frac{1}{2}} d k_{t} d k_{t-1} \\
& \cdot \int\left\{\sum_{i=1}^{n}\left[\left(\int b_{1}\left(\mathbf{y}_{t}\right) \hat{f}_{\mathbf{y}_{t} \mid \mathbf{x}_{t i}} d \mathbf{y}_{t} \hat{f}_{k_{t}, k_{t-1} \mid \mathbf{s}_{t i}}^{\frac{1}{2}}\right)^{2}+\left(\int b_{2}\left(\mathbf{y}_{t, t-1}\right) \hat{f}_{\mathbf{y}_{t, t-1} \mid \tilde{\mathbf{x}}_{t i}} d \mathbf{y}_{t, t-1} \hat{f}_{k_{t}, k_{t-1} \mid \mathbf{s}_{t i}}^{\frac{1}{2}}\right)^{2}\right]\right\}^{1 / 2} \\
& \left.\cdot\|\tilde{\theta}-\theta\|_{s, \theta}^{\nu} d k_{t} d k_{t-1}\right\} \\
& \leq B_{2 n}\|\tilde{\theta}-\theta\|_{s, \theta}^{\nu},
\end{aligned}
$$


where $B_{2 n} \equiv \tilde{C} \tilde{B}_{n}$ and by Assumption 5.7, we have

$$
\begin{aligned}
& \tilde{B}_{n} \\
\equiv & \frac{1}{n}\left\{\int \sum_{i=1}^{n}\left[\left(\int b_{1}\left(\mathbf{y}_{t}\right) \hat{f}_{\mathbf{y}_{t} \mid \mathbf{x}_{t i}} d \mathbf{y}_{t} \hat{f}_{k_{t}, k_{t-1} \mid \mathbf{s}_{t i}}^{\frac{1}{2}}\right)^{2}+\left(\int b_{2}\left(\mathbf{y}_{t, t-1}\right) \hat{f}_{\mathbf{y}_{t, t-1} \mid \tilde{\mathbf{x}}_{t i}} d \mathbf{y}_{t, t-1} \hat{f}_{k_{t}, k_{t-1} \mid \mathbf{s}_{t i}}^{\frac{1}{2}}\right)^{2}\right] d k_{t} d k_{t-1}\right. \\
& +2 \int\left\{\sum _ { i = 1 } ^ { n } \left[\left(\int\left(\rho_{1}\left(\mathbf{y}_{t} ; \beta_{0}\right)\right) \hat{f}_{\mathbf{y}_{t} \mid \mathbf{x}_{t i}} d \mathbf{y}_{t}\right)^{2}\right.\right. \\
& \left.\left.+\left(\int \rho_{2}\left(\mathbf{y}_{t, t-1} ; \theta_{0}\right) \hat{f}_{\mathbf{y}_{t, t-1} \mid \tilde{\mathbf{x}}_{t i}} d \mathbf{y}_{t, t-1}\right)^{2}\right]\right\}^{1 / 2} \hat{f}_{k_{t}, k_{t-1} \mid \mathbf{s}_{t i}}^{\frac{1}{2}} d k_{t} d k_{t-1} \\
& \cdot \int\left\{\sum_{i=1}^{n}\left[\left(\int b_{1}\left(\mathbf{y}_{t}\right) \hat{f}_{\mathbf{y}_{t} \mid \mathbf{x}_{t i}} d \mathbf{y}_{t} \hat{f}_{k_{t}, k_{t-1} \mid \mathbf{s}_{t i}}^{\frac{1}{2}}\right)^{2}+\left(\int b_{2}\left(\mathbf{y}_{t, t-1}\right) \hat{f}_{\mathbf{y}_{t, t-1} \mid \tilde{\mathbf{x}}_{t i}} d \mathbf{y}_{t, t-1} \hat{f}_{k_{t}, k_{t-1} \mid \mathbf{s}_{t i}}^{\frac{1}{2}}\right)^{2}\right]\right\}^{1 / 2} d k_{t} d k_{t-1} \\
= & O_{p}(1) .
\end{aligned}
$$

Therefore, we satisfy Condition (iii) of Lemma A.2. We have verified all three conditions in Lemma A.1 of Newey and Powell (2003) and hence the result follows.

\section{C.2 Convergence Rate: Proof of Theorem 5.21}

The first result (Theorem 5.21.1) follows from a similar argument to Theorem 2 in Hu and Schennach (2008). The second result (Theorem 5.21.2) follows from a similar argument to Ai and Chen (2003) and Song (2012)'s convergence rate results. First we show two lemmas: Lemma C.1 and Lemma C.2 below, which are useful to prove Theorem 5.21.

Lemma C.1. Suppose Assumptions for Theorem 5.10 hold. Then (a) under Assumptions 5.4, 5.7, 5.11, 5.12, 5.13, 5.16, 5.17, 5.18, 5.20, $\sum_{i=1}^{n}\left[\int\left\|\hat{g}\left(\mathbf{x}_{t i} ; \theta\right) \hat{f}_{k_{t}, k_{t-1} \mid \mathbf{s}_{t i}}^{\frac{1}{2}}-g\left(\mathbf{x}_{t i} ; \theta\right) f_{k_{t}, k_{t-1} \mid \mathbf{s}_{t i}}^{\frac{1}{2}}\right\|_{E} d k_{t} d k_{t-1}\right]^{2} / n$ $=o_{p}\left(n^{-1 / 2}\right)$ uniformly over $\theta \in \Theta ;(b)$ Under Assumptions 5.5 and 5.7, $\sum_{i=1}^{n} \int\left\|\hat{g}\left(\mathbf{x}_{t i} ; \theta_{0}\right) \hat{f}_{k_{t}, k_{t-1} \mid \mathbf{s}_{t i}}^{\frac{1}{2}}\right\|_{E}^{2} d k_{t} d k_{t-1} / n=O_{p}\left(\kappa_{1 n} / n\right)$.

Proof. (a) Since $\left\|\hat{\alpha}-\alpha_{0}\right\|_{\alpha}=o_{p}\left(n^{-1 / 4}\right)$ by a similar argument to Theorem 2 in Hu and Schennach (2008), $\left\|\hat{f}_{k_{t}, k_{t-1} \mid \mathbf{s}_{t}}-f_{k_{t}, k_{t-1} \mid \mathbf{s}_{t}}\right\|_{\alpha}^{2}=o_{p}\left(n^{-1 / 2}\right)$. From

$$
\begin{aligned}
& \sum_{i=1}^{n}\left[\int\left\|\hat{g}\left(\mathbf{x}_{t i} ; \theta\right) \hat{f}_{k_{t}, k_{t-1} \mid \mathbf{s}_{t i}}^{\frac{1}{2}}-g\left(\mathbf{x}_{t i} ; \theta\right) f_{k_{t}, k_{t-1} \mid \mathbf{s}_{t i}}^{\frac{1}{2}}\right\|_{E} d k_{t} d k_{t-1}\right]^{2} / n \\
\leq & C \cdot\left(\sum_{i=1}^{n}\left[\int\left\|\hat{g}\left(\mathbf{x}_{t i} ; \theta\right)-g\left(\mathbf{x}_{t i} ; \theta\right)\right\|_{E} d k_{t} d k_{t-1}\right]^{2}+\left\|\hat{f}_{k_{t}, k_{t-1} \mid \mathbf{s}_{t i}}-f_{k_{t}, k_{t-1} \mid \mathbf{s}_{t i}}\right\|_{\alpha}^{2}\right) / n+o_{p}\left(n^{-1 / 2}\right),
\end{aligned}
$$

for a generic constant $C$, it suffices to show $\sum_{i=1}^{n}\left[\int\left\|\hat{g}\left(\mathbf{x}_{t i} ; \theta\right)-g\left(\mathbf{x}_{t i} ; \theta\right)\right\|_{E} d k_{t} d k_{t-1}\right]^{2} / n=o_{p}\left(n^{-1 / 2}\right)$ uniformly over $\theta \in \Theta$. We note that from Corollary 4.4, equation (12), and Assumption 5.20, $\left\|\hat{f}_{y_{t} \mid k_{t}, k_{t-1}, m_{t-1}, l_{t-1}}-f_{y_{t} \mid k_{t}, k_{t-1}, m_{t-1}, l_{t-1}}\right\|_{\alpha}=C\left\|\hat{f}_{y_{t} \mid k_{t}, k_{t-1}, \mathbf{s}_{t}}-f_{y_{t} \mid k_{t}, k_{t-1}, \mathbf{s}_{t}}\right\|_{\alpha}+o_{p}\left(n^{-1 / 4}\right)$ for a generic 
constant $C$. We also note that $\left\|\hat{f}_{r_{t} \mid k_{t}, k_{t-1}, m_{t-1}, l_{t-1}}-f_{r_{t} \mid k_{t}, k_{t-1}, m_{t-1}, l_{t-1}}\right\|_{\alpha} \asymp \| \hat{f}_{y_{t} \mid k_{t}, k_{t-1}, m_{t-1}, l_{t-1}}-$ $f_{y_{t} \mid k_{t}, k_{t-1}, m_{t-1}, l_{t-1}} \|_{\alpha}$ for $r_{t} \in\left\{l_{t}, m_{t}\right\}$. It follows that

$$
\begin{aligned}
& E \sum_{i=1}^{n}\left[\int\left\|\hat{g}\left(\mathbf{x}_{t i} ; \theta\right)-g\left(\mathbf{x}_{t i} ; \theta\right)\right\|_{E} d k_{t} d k_{t-1}\right]^{2} / n \\
& =\frac{1}{n} \sum_{i=1}^{n} \sum_{j=1}^{2} E\left(\int\left\{\int \rho_{j}\left(\mathbf{y}_{t, t-1} ; \theta\right) \hat{f}_{\mathbf{y}_{t, t-1} \mid \mathbf{x}_{t i}} d \mathbf{y}_{t, t-1}-\int \rho_{j}\left(\mathbf{y}_{t, t-1} ; \theta\right) f_{\mathbf{y}_{t, t-1} \mid \mathbf{x}_{t i}} d \mathbf{y}_{t, t-1}\right\} d k_{t} d k_{t-1}\right)^{2} \\
& =\frac{1}{n} \sum_{i=1}^{n} E\left[\left(\int \left\{\left(\int y_{t} \hat{f}_{y_{t} \mid k_{t}, k_{t-1}, \mathbf{s}_{t i}} d y_{t}-\beta_{l} l_{t i}-\beta_{k} k_{t}-\beta_{m} m_{t i}-\mathbf{c}\left(m_{t i}, k_{t}\right)^{\prime} \beta_{\omega}\right)\right.\right.\right. \\
& \left.\left.-\left(\int y_{t} f_{y_{t} \mid k_{t}, k_{t-1}, \mathbf{s}_{t i}} d y_{t}-\beta_{l} l_{t i}-\beta_{k} k_{t}-\beta_{m} m_{t i}-\mathbf{c}\left(m_{t i}, k_{t}\right)^{\prime} \beta_{\omega}\right)\right\} d k_{t} d k_{t-1}\right)^{2} \\
& +\left(\int \left\{\left(\int y_{t} \hat{f}_{y_{t} \mid k_{t}, k_{t-1}, m_{t-1, i}, l_{t-1, i}} d y_{t}-\beta_{l} \int l_{t} \hat{f}_{l_{t} \mid k_{t}, k_{t-1}, m_{t-1, i}, l_{t-1, i}} d l_{t}-\beta_{k} k_{t}\right.\right.\right. \\
& \left.-\beta_{m} \int m_{t} \hat{f}_{m_{t} \mid k_{t}, k_{t-1}, m_{t-1, i}, l_{t-1, i}} d m_{t}-q\left(\mathbf{c}\left(m_{t-1, i}, k_{t-1}\right)^{\prime} \beta_{\omega}\right)\right) \\
& -\left(\int y_{t} f_{y_{t} \mid k_{t}, k_{t-1}, m_{t-1, i}, l_{t-1, i}} d y_{t}-\beta_{l} \int l_{t} f_{l_{t} \mid k_{t}, k_{t-1}, m_{t-1, i}, l_{t-1, i}} d l_{t}-\beta_{k} k_{t}\right. \\
& \left.\left.\left.\left.-\beta_{m} \int m_{t} f_{m_{t} \mid k_{t}, k_{t-1}, m_{t-1, i}, l_{t-1, i}} d m_{t}-q\left(\mathbf{c}\left(m_{t-1, i}, k_{t-1}\right)^{\prime} \beta_{\omega}\right)\right)\right\} d k_{t} d k_{t-1}\right)^{2}\right] \\
& =\frac{1}{n} \sum_{i=1}^{n} E\left\{\left(\int y_{t}\left(\hat{f}_{y_{t} \mid k_{t}, k_{t-1}, \mathbf{s}_{t i}}-f_{y_{t} \mid k_{t}, k_{t-1}, \mathbf{s}_{t i}}\right) d y_{t} d k_{t} d k_{t-1}\right)^{2}\right. \\
& +\left(\int y_{t}\left(\hat{f}_{y_{t} \mid k_{t}, k_{t-1}, m_{t-1, i}, l_{t-1, i}}-f_{y_{t} \mid k_{t}, k_{t-1}, m_{t-1, i}, l_{t-1, i}}\right) d y_{t} d k_{t} d k_{t-1}\right. \\
& -\beta_{l} \int l_{t}\left(\hat{f}_{l_{t} \mid k_{t}, k_{t-1}, m_{t-1, i}, l_{t-1, i}}-f_{l_{t} \mid k_{t}, k_{t-1}, m_{t-1, i}, l_{t-1, i}}\right) d l_{t} d k_{t} d k_{t-1} \\
& \left.\left.-\beta_{m} \int m_{t}\left(\hat{f}_{m_{t} \mid k_{t}, k_{t-1}, m_{t-1, i}, l_{t-1, i}}-f_{m_{t} \mid k_{t}, k_{t-1}, m_{t-1, i}, l_{t-1, i}}\right) d m_{t} d k_{t} d k_{t-1}\right)^{2}\right\} \\
& \leq \frac{C}{n} \sum_{i=1}^{n} E\left\{\left(\int y_{t}\left(\hat{f}_{y_{t} \mid k_{t}, k_{t-1}, \mathbf{s}_{t i}}-f_{y_{t} \mid k_{t}, k_{t-1}, \mathbf{s}_{t i}}\right) d y_{t} d k_{t} d k_{t-1}\right)^{2}\right\}+o_{p}\left(n^{-1 / 2}\right) \\
& =\frac{C}{n} \sum_{i=1}^{n} E\left\{\left(\int y_{t}\left(f_{k_{t}^{*}, k_{t-1}^{*} \mid \mathbf{z}_{t, i}} f_{k_{t}, k_{t-1} \mid \mathbf{z}_{t, i}^{*}}\right)^{-1}\right.\right. \\
& \left.\left.\cdot \frac{d}{d \psi} f_{y_{t} \mid \mathbf{z}_{t, i} ; \psi_{0}}\left[\hat{\psi}-\psi_{0}\right] f_{k_{t}^{*}, k_{t-1}^{*} \mid \mathbf{z}_{t, i}} f_{k_{t}, k_{t-1} \mid \mathbf{z}_{t, i}^{*}} d y_{t} d k_{t} d k_{t-1}\right)^{2}\right\}+o_{p}\left(n^{-1 / 2}\right) \\
& \leq \frac{C}{n} \sum_{i=1}^{n} E\left\{\int y_{t}\left(f_{k_{t}^{*}, k_{t-1}^{*} \mid \mathbf{z}_{t, i}} f_{k_{t}, k_{t-1} \mid \mathbf{z}_{t, i}^{*}}\right)^{-1}\right. \\
& \left.\cdot\left(\int \frac{d}{d \psi} f_{y_{t} \mid \mathbf{z}_{t, i} ; \psi_{0}}\left[\hat{\psi}-\psi_{0}\right] f_{k_{t}^{*}, k_{t-1}^{*} \mid \mathbf{z}_{t, i}} f_{k_{t}, k_{t-1} \mid \mathbf{z}_{t, i}^{*}} d k_{t} d k_{t-1}\right)^{2} d y_{t}\right\}+o_{p}\left(n^{-1 / 2}\right)
\end{aligned}
$$




$$
\begin{aligned}
& =\frac{C}{n} \sum_{i=1}^{n} \int \sup _{\left\{k_{t}^{*}, k_{t-1}^{*}, \mathbf{z}_{t}^{*}, \mathbf{z}_{t}\right\}}\left\{y_{t}\left(f_{k_{t}^{*}, k_{t-1}^{*} \mid \mathbf{z}_{t, i}} f_{k_{t}, k_{t-1} \mid \mathbf{z}_{t, i}^{*}}\right)^{-1} f_{y_{t}, k_{t}^{*}, k_{t-1}^{*} \mid \mathbf{z}_{t, i}^{*} ; \alpha_{0}}\right\} \\
& \cdot E\left\{\left(\frac{1}{f_{y_{t}, k_{t}^{*}, k_{t-1}^{*} \mid \mathbf{z}_{t, i}^{*} ; \alpha_{0}}} \int \frac{d}{d \psi} f_{y_{t} \mid \mathbf{z}_{t, i} ; \psi_{0}}\left[\hat{\psi}-\psi_{0}\right] f_{k_{t}^{*}, k_{t-1}^{*} \mid \mathbf{z}_{t, i}} f_{k_{t}, k_{t-1} \mid \mathbf{z}_{t, i}^{*}} d k_{t} d k_{t-1}\right)^{2}\right\} d y_{t}+o_{p}\left(n^{-1 / 2}\right) \\
& =\frac{C}{n} \sum_{i=1}^{n} \int \sup _{\left\{k_{t}^{*}, k_{t-1}^{*}, \mathbf{z}_{t}^{*}, \mathbf{z}_{t}\right\}}\left\{y_{t}\left(f_{k_{t}^{*}, k_{t-1}^{*} \mid \mathbf{z}_{t, i}} f_{k_{t}, k_{t-1} \mid \mathbf{z}_{t, i}^{*}}\right)^{-1} f_{y_{t}, k_{t}^{*}, k_{t-1}^{*} \mid \mathbf{z}_{t, i}^{*} ; \alpha_{0}}\right\}
\end{aligned}
$$

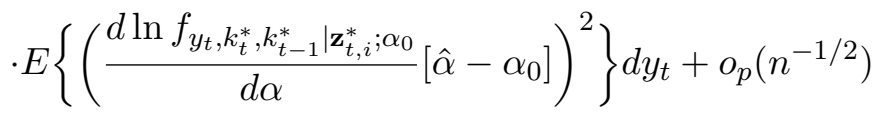

$$
\begin{aligned}
& \leq \frac{C}{n} \sum_{i=1}^{n} \int \sup _{\left\{k_{t}^{*}, k_{t-1}^{*}, \mathbf{z}_{t}^{*}, \mathbf{z}_{t}\right\}}\left\{y_{t}\left(f_{k_{t}^{*}, k_{t-1}^{*} \mid \mathbf{z}_{t, i}} f_{k_{t}, k_{t-1} \mid \mathbf{z}_{t, i}^{*}}\right)^{-1} f_{y_{t}, k_{t}^{*}, k_{t-1}^{*} \mid \mathbf{z}_{t, i}^{*} ; \alpha_{0}}\right\} d y_{t}\left\|\hat{\alpha}-\alpha_{0}\right\|_{\alpha}^{2}+o_{p}\left(n^{-1 / 2}\right) \\
& =o_{p}\left(n^{-1 / 2}\right) \text {, }
\end{aligned}
$$

where the last result holds since $\int \sup _{\left\{k_{t}^{*}, k_{t-1}^{*}, \mathbf{z}_{t}^{*}, \mathbf{z}_{t}\right\}}\left\{y_{t}\left(f_{k_{t}^{*}, k_{t-1}^{*} \mid \mathbf{z}_{t, i}} f_{k_{t}, k_{t-1} \mid \mathbf{z}_{t, i}^{*}}\right)^{-1} f_{y_{t}, k_{t}^{*}, k_{t-1}^{*} \mid \mathbf{z}_{t, i}^{*} ; \alpha_{0}}\right\} d y_{t}=$ $O(1)$. Thus the result follows by the Markov inequality.

(b) The result follows from Corollary A.1 (ii) in Ai and Chen (2003).

Lemma C.2. (a) Under Assumptions 5.1, 5.3, 5.5, 5.6.2, 5.11, 5.13.1, and 5.19, we obtain $\sum_{i=1}^{n}\left\|g\left(\mathbf{x}_{t i} ; \theta\right)\right\|_{E}^{2} / n-E\left[\left\|g\left(\mathbf{x}_{t i} ; \theta\right)\right\|_{E}^{2}\right]=o_{p}\left(n^{-1 / 2}\right)$ uniformly over $\theta \in\left\{\Theta_{n}:\left\|\theta-\theta_{0}\right\|_{\theta}=o(1)\right\} ;$ (b) Under Assumptions 5.1, 5.3, 5.5, 5.6, 5.7, 5.9, 5.11, 5.13, 5.15, 5.18, 5.19, we obtain $\sum_{i=1}^{n}\left\|\hat{g}\left(\mathbf{x}_{t i} ; \theta\right)\right\|_{E}^{2} / n=$ $o_{p}\left(\eta_{n}^{2}\right)$ and $\sum_{i=1}^{n}\left\|g\left(\mathbf{x}_{t i} ; \theta\right)\right\|_{E}^{2} / n=o_{p}\left(\eta_{n}^{2}\right)$ uniformly over $\theta \in\left\{\Theta_{n}:\left\|\theta-\theta_{0}\right\|_{\theta}=o\left(\eta_{n}\right)\right\}$, where $\eta_{n}=n^{-\tau}$ with $\tau \leq 1 / 4$.

Proof. (a) The result follows from Corollary A.2 (i) in Ai and Chen (2003).

(b) Note that $E\left[\left\|g\left(\mathbf{x}_{t i} ; \theta\right)\right\|_{E}^{2}\right]=o\left(\eta_{n}^{2}\right)$ by Assumptions 5.6.2 and 5.19. Then the result follows from applying Lemma C.1 (a) and Lemma C.2 (a).

Next we prove Theorem 5.21.

Proof. 1. The result follows from a similar argument to the proof of Theorem 2 in $\mathrm{Hu}$ and Schennach (2008).

2. From Lemma C.1 (a) and Assumption 5.6, we obtain $\widehat{Q}_{n}(\theta)-Q_{n}(\theta)=o_{p}\left(n^{-1 / 4}\right)$ uniformly over $\theta \in \Theta_{n}$. In addition, from Lemma C.1 and Lemma C.2 (b), we obtain $\widehat{Q}_{n}(\theta)-\widehat{Q}_{n}\left(\theta_{0}\right)-$ $\left\{Q_{n}(\theta)-Q_{n}\left(\theta_{0}\right)\right\}=o_{p}\left(\eta_{n} n^{-1 / 4}\right)$ uniformly over $\theta \in\left\{\Theta_{n}:\left\|\theta-\theta_{0}\right\|_{\theta}=o\left(\eta_{n}\right)\right\}$, where $\eta_{n}=n^{-\tau}$ with $\tau \leq 1 / 4$. Then the result follows by a similar argument to the proof of Theorem $3.1 \mathrm{in} \mathrm{Ai}$ and Chen (2003). 


\section{C.3 Asymptotic Normality: Proof of Theorem 6.12}

The first result (Theorem 6.12.1) follows from a similar argument to Theorem 3 in $\mathrm{Hu}$ and Schennach (2008). The second result (Theorem 6.12.2) follows from a similar argument to Ai and Chen (2003) and Song (2012)'s asymptotic normality results. First we show two lemmas: Lemma C.3 and Lemma C.4 below, which are useful to prove Theorem 6.12 .

Define

$$
\begin{aligned}
& \frac{d Q_{n}(\theta)}{d \theta}\left[v_{2 n}^{*}\right]=\frac{1}{n} \sum_{i=1}^{n} \int\left\{\frac{d g\left(\mathbf{x}_{t i} ; \theta\right)}{d \theta}\left[v_{2 n}^{*}\right]\right\}^{\prime} A\left(\mathbf{x}_{t i}\right) g\left(\mathbf{x}_{t i} ; \theta\right) f_{k_{t}, k_{t-1} \mid \mathbf{s}_{t i}} d k_{t} d k_{t-1} \\
& \frac{d \widehat{Q}_{n}(\theta)}{d \theta}\left[v_{2 n}^{*}\right]=\frac{1}{n} \sum_{i=1}^{n} \int\left\{\frac{d \hat{g}\left(\mathbf{x}_{t i} ; \theta\right)}{d \theta}\left[v_{2 n}^{*}\right]\right\}^{\prime} \hat{A}\left(\mathbf{x}_{t i}\right) \hat{g}\left(\mathbf{x}_{t i} ; \theta\right) \hat{f}_{k_{t}, k_{t-1} \mid \mathbf{s}_{t i}} d k_{t} d k_{t-1}
\end{aligned}
$$

and similarly define

$$
\begin{aligned}
& \frac{d^{2} Q_{1 n}(\theta)}{d \theta d \theta}\left[v_{2 n}^{*}, v_{2 n}^{*}\right]=\frac{1}{n} \sum_{i=1}^{n} \int\left\{\frac{d g\left(\mathbf{x}_{t i} ; \theta\right)}{d \theta}\left[v_{2 n}^{*}\right]\right\}^{\prime} A\left(\mathbf{x}_{t i}\right)\left\{\frac{d g\left(\mathbf{x}_{t i} ; \theta\right)}{d \theta}\left[v_{2 n}^{*}\right]\right\} f_{k_{t}, k_{t-1} \mid \mathbf{s}_{t i}} d k_{t} d k_{t-1}, \\
& \frac{d^{2} \widehat{Q}_{1 n}(\theta)}{d \theta d \theta}\left[v_{2 n}^{*}, v_{2 n}^{*}\right]=\frac{1}{n} \sum_{i=1}^{n} \int\left\{\frac{d \hat{g}\left(\mathbf{x}_{t i} ; \theta\right)}{d \theta}\left[v_{2 n}^{*}\right]\right\}^{\prime} \hat{A}\left(\mathbf{x}_{t i}\right)\left\{\frac{d \hat{g}\left(\mathbf{x}_{t i} ; \theta\right)}{d \theta}\left[v_{2 n}^{*}\right]\right\} \hat{f}_{k_{t}, k_{t-1} \mid \mathbf{s}_{t i}} d k_{t} d k_{t-1}, \\
& \frac{d^{2} Q_{2 n}(\theta)}{d \theta d \theta}\left[v_{2 n}^{*}, v_{2 n}^{*}\right]=\frac{1}{n} \sum_{i=1}^{n} \int\left\{\frac{d^{2} g\left(\mathbf{x}_{t i} ; \theta\right)}{d \theta d \theta}\left[v_{2 n}^{*}, v_{2 n}^{*}\right]\right\}^{\prime} A\left(\mathbf{x}_{t i}\right) g\left(\mathbf{x}_{t i} ; \theta\right) f_{k_{t}, k_{t-1} \mid \mathbf{s}_{t i}} d k_{t} d k_{t-1}, \\
& \frac{d^{2} \widehat{Q}_{2 n}(\theta)}{d \theta d \theta}\left[v_{2 n}^{*}, v_{2 n}^{*}\right]=\frac{1}{n} \sum_{i=1}^{n} \int\left\{\frac{d^{2} \hat{g}\left(\mathbf{x}_{t i} ; \theta\right)}{d \theta d \theta}\left[v_{2 n}^{*}, v_{2 n}^{*}\right]\right\}^{\prime} \hat{A}\left(\mathbf{x}_{t i}\right) \hat{g}\left(\mathbf{x}_{t i} ; \theta\right) \hat{f}_{k_{t}, k_{t-1} \mid \mathbf{s}_{t i}} d k_{t} d k_{t-1},
\end{aligned}
$$

where

$$
\begin{aligned}
\frac{d g\left(\mathbf{x}_{t} ; \theta\right)}{d \theta}\left[v_{2 n}^{*}\right] & =\int \frac{d \rho\left(\mathbf{y}_{t, t-1} ; \theta\right)}{d \theta}\left[v_{2 n}^{*}\right] f_{\mathbf{y}_{t, t-1} \mid \mathbf{x}_{t}} d \mathbf{y}_{t, t-1}, \\
\frac{d \hat{g}\left(\mathbf{x}_{t} ; \theta\right)}{d \theta}\left[v_{2 n}^{*}\right] & =\int \frac{d \rho\left(\mathbf{y}_{t, t-1} ; \theta\right)}{d \theta}\left[v_{2 n}^{*}\right] \hat{f}_{\mathbf{y}_{t, t-1} \mid \mathbf{x}_{t}} d \mathbf{y}_{t, t-1}, \\
\frac{d^{2} g\left(\mathbf{x}_{t} ; \theta\right)}{d \theta d \theta}\left[v_{2 n}^{*}, v_{2 n}^{*}\right] & =\int \frac{d^{2} \rho\left(\mathbf{y}_{t, t-1} ; \theta\right)}{d \theta d \theta}\left[v_{2 n}^{*}, v_{2 n}^{*}\right] f_{\mathbf{y}_{t, t-1} \mid \mathbf{x}_{t}} d \mathbf{y}_{t, t-1}, \\
\frac{d^{2} \hat{g}\left(\mathbf{x}_{t} ; \theta\right)}{d \theta d \theta}\left[v_{2 n}^{*}, v_{2 n}^{*}\right] & =\int \frac{d^{2} \rho\left(\mathbf{y}_{t, t-1} ; \theta\right)}{d \theta d \theta}\left[v_{2 n}^{*}, v_{2 n}^{*}\right] \hat{f}_{\mathbf{y}_{t, t-1} \mid \mathbf{x}_{t}} d \mathbf{y}_{t, t-1} .
\end{aligned}
$$

Lemma C.3. (a) Under Assumptions 5.1, 5.3-5.4, 5.6-5.7, 5.9, 6.6.2-6.9, we have

$$
\sup _{\tilde{\theta} \in \mathcal{N}_{02 n}} \frac{d^{2} \widehat{Q}_{1 n}(\tilde{\theta})}{d \theta d \theta}\left[v_{2 n}^{*}, v_{2 n}^{*}\right]=\frac{d^{2} Q_{1 n}\left(\theta_{0}\right)}{d \theta d \theta}\left[v_{2 n}^{*}, v_{2 n}^{*}\right]+o_{p}\left(n^{-1 / 4}\right) .
$$


(b) Under Assumptions 5.1-5.4, 5.6-5.9, 5.11-5.20, 6.11, we have

$$
\sup _{\tilde{\theta} \in \mathcal{N}_{02 n}} \frac{d^{2} \widehat{Q}_{2 n}(\tilde{\theta})}{d \theta d \theta}\left[v_{2 n}^{*}, v_{2 n}^{*}\right]=o_{p}\left(n^{-1 / 4}\right) .
$$

Proof. (a) We have that uniformly over $\tilde{\theta} \in \mathcal{N}_{02 n}$,

$$
\begin{aligned}
& \frac{d^{2} \widehat{Q}_{1 n}(\tilde{\theta})}{d \theta d \theta}\left[v_{2 n}^{*}, v_{2 n}^{*}\right]-\frac{d^{2} Q_{1 n}\left(\theta_{0}\right)}{d \theta d \theta}\left[v_{2 n}^{*}, v_{2 n}^{*}\right] \\
& =\frac{1}{n} \sum_{i=1}^{n} \int\left[\left\{\frac{d \hat{g}\left(\mathbf{x}_{t i} ; \theta\right)}{d \theta}\left[v_{2 n}^{*}\right]-\frac{d g\left(\mathbf{x}_{t i} ; \theta_{0}\right)}{d \theta}\left[v_{2 n}^{*}\right]\right\}^{\prime} \hat{A}\left(\mathbf{x}_{t i}\right)\left\{\frac{d \hat{g}\left(\mathbf{x}_{t i} ; \theta\right)}{d \theta}\left[v_{2 n}^{*}\right]\right\} \hat{f}_{k_{t}, k_{t-1} \mid \mathbf{s}_{t i}}\right. \\
& +\left\{\frac{d g\left(\mathbf{x}_{t i} ; \theta_{0}\right)}{d \theta}\left[v_{2 n}^{*}\right]\right\}^{\prime}\left\{\hat{A}\left(\mathbf{x}_{t i}\right)-A\left(\mathbf{x}_{t i}\right)\right\}\left\{\frac{d \hat{g}\left(\mathbf{x}_{t i} ; \theta\right)}{d \theta}\left[v_{2 n}^{*}\right]\right\} \hat{f}_{k_{t}, k_{t-1} \mid \mathbf{s}_{t i}} \\
& +\left\{\frac{d g\left(\mathbf{x}_{t i} ; \theta_{0}\right)}{d \theta}\left[v_{2 n}^{*}\right]\right\}^{\prime} A\left(\mathbf{x}_{t i}\right)\left\{\frac{d \hat{g}\left(\mathbf{x}_{t i} ; \theta\right)}{d \theta}\left[v_{2 n}^{*}\right]-\frac{d g\left(\mathbf{x}_{t i} ; \theta\right)}{d \theta}\left[v_{2 n}^{*}\right]\right\} \hat{f}_{k_{t}, k_{t-1} \mid \mathbf{s}_{t i}} \\
& \left.+\left\{\frac{d g\left(\mathbf{x}_{t i} ; \theta_{0}\right)}{d \theta}\left[v_{2 n}^{*}\right]\right\}^{\prime} A\left(\mathbf{x}_{t i}\right)\left\{\frac{d g\left(\mathbf{x}_{t i} ; \theta_{0}\right)}{d \theta}\left[v_{2 n}^{*}\right]\right\}\left\{\hat{f}_{k_{t}, k_{t-1} \mid \mathbf{s}_{t i}}-f_{k_{t}, k_{t-1} \mid \mathbf{s}_{t i}}\right\}\right] d k_{t} d k_{t-1} \\
& \equiv A_{1}+A_{2}+A_{3}+A_{4} \text {. }
\end{aligned}
$$

Since

$$
\begin{aligned}
& E\left[\frac{1}{n} \sum_{i=1}^{n}\left\|\frac{d \hat{g}\left(\mathbf{x}_{t i} ; \theta\right)}{d \theta}\left[v_{2 n}^{*}\right]-\frac{d g\left(\mathbf{x}_{t i} ; \theta\right)}{d \theta}\left[v_{2 n}^{*}\right]\right\|_{E}^{2}\right] \\
= & \frac{1}{n} \sum_{i=1}^{n} \sum_{j=1}^{2} E\left[\left(\int \frac{d \rho_{j}\left(\mathbf{y}_{t, t-1} ; \theta\right)}{d \theta}\left[v_{2 n}^{*}\right]\left(\hat{f}_{\mathbf{y}_{t, t-1} \mid \mathbf{x}_{t i}}-f_{\mathbf{y}_{t, t-1} \mid \mathbf{x}_{t i}}\right) d \mathbf{y}_{t, t-1}\right)^{2}\right] \\
= & o\left(n^{-1 / 2}\right),
\end{aligned}
$$

uniformly over $\tilde{\theta} \in \mathcal{N}_{02 n}$ from $\left\|\hat{\alpha}-\alpha_{0}\right\|_{\alpha}=o_{p}\left(n^{-1 / 4}\right)$, we obtain $A_{1}=A_{3}=A_{4}=o_{p}\left(n^{-1 / 4}\right)$ by the Markov inequality, Assumption 5.6, and Lemma C.2 (b). Also we obtain $A_{2}=o_{p}\left(n^{-1 / 4}\right)$ from Assumption 5.15 and Lemma C.2 (b).

(b) By Assumption 5.6 and Lemma C.2 (b), we obtain that for some generic constant $C$,

$$
\begin{aligned}
& \left|\frac{d^{2} \widehat{Q}_{2 n}(\tilde{\theta})}{d \theta d \theta}\left[v_{2 n}^{*}, v_{2 n}^{*}\right]\right| \\
\leq & C \quad \sqrt{\frac{1}{n} \sum_{i=1}^{n} \int\left\|\frac{d^{2} \hat{g}\left(\mathbf{x}_{t i} ; \tilde{\theta}\right)}{d \theta d \theta}\left[v_{2 n}^{*}, v_{2 n}^{*}\right]\right\|_{E}^{2} d k_{t} d k_{t-1}} \cdot \sqrt{\frac{1}{n} \sum_{i=1}^{n} \int\left\|\hat{g}\left(\mathbf{x}_{t i} ; \tilde{\theta}\right)\right\|_{E}^{2} d k_{t} d k_{t-1}} \\
= & o_{p}\left(n^{-1 / 4}\right),
\end{aligned}
$$

since Assumption 6.11 implies that uniformly over $\tilde{\theta} \in \mathcal{N}_{02 n}$, for a measurable function $c\left(\mathbf{y}_{t, t-1}\right)$ 
with bounded second moment,

$$
\left\|\frac{d^{2} \hat{g}\left(\mathbf{x}_{t i} ; \tilde{\theta}\right)}{d \theta d \theta}\left[v_{2 n}^{*}, v_{2 n}^{*}\right]\right\|_{E}^{2} \leq \frac{1}{n} \sum_{i=1}^{n} c\left(\mathbf{y}_{t, t-1, i}\right)^{2}=O_{p}(1) .
$$

Lemma C.4. (a) Under Assumptions 5.1-5.9, 6.6.2-6.9, we have uniformly over $\tilde{\theta} \in \mathcal{N}_{02 n}$,

$$
\begin{aligned}
& \frac{d \widehat{Q}_{n}(\tilde{\theta})}{d \theta}\left[v_{2 n}^{*}\right] \\
= & \frac{1}{n} \sum_{i=1}^{n} \int\left\{\frac{d g\left(\mathbf{x}_{t i} ; \theta_{0}\right)}{d \theta}\left[v_{2}^{*}\right]\right\}^{\prime} A\left(\mathbf{x}_{t i}\right) \hat{g}\left(\mathbf{x}_{t i} ; \tilde{\theta}\right) f_{k_{t}, k_{t-1} \mid \mathbf{s}_{t i}} d k_{t} d k_{t-1} .
\end{aligned}
$$

(b) Under Assumptions 5.1, 5.3, 5.12, 6.6.2-6.9, we have uniformly over $\tilde{\theta} \in \mathcal{N}_{02 n}$,

$$
\begin{aligned}
& \frac{1}{n} \sum_{i=1}^{n} \int\left\{\frac{d g\left(\mathbf{x}_{t i} ; \theta_{0}\right)}{d \theta}\left[v_{2}^{*}\right]\right\}^{\prime} A\left(\mathbf{x}_{t i}\right)\left\{\hat{g}\left(\mathbf{x}_{t i} ; \tilde{\theta}\right)-\hat{g}\left(\mathbf{x}_{t i} ; \theta_{0}\right)\right\} f_{k_{t}, k_{t-1} \mid \mathbf{s}_{t i}} d k_{t} d k_{t-1} \\
= & \left\langle v_{2}^{*}, \tilde{\theta}-\theta_{0}\right\rangle_{\theta}+o_{p}\left(n^{-1 / 2}\right) .
\end{aligned}
$$

(c) Under Assumptions 5.1, 5.3, 5.4, 5.6.2, 5.12, 5.16, 6.6.3, we have

$$
\begin{aligned}
& \frac{1}{n} \sum_{i=1}^{n} \int\left\{\frac{d g\left(\mathbf{x}_{t i} ; \theta_{0}\right)}{d \theta}\left[v_{2}^{*}\right]\right\}^{\prime} A\left(\mathbf{x}_{t i}\right) \hat{g}\left(\mathbf{x}_{t i} ; \theta_{0}\right) f_{k_{t}, k_{t-1} \mid \mathbf{s}_{t i}} d k_{t} d k_{t-1} \\
= & \frac{1}{n} \sum_{i=1}^{n} \int\left\{\frac{d g\left(\mathbf{x}_{t i} ; \theta_{0}\right)}{d \theta}\left[v_{2}^{*}\right]\right\}^{\prime} A\left(\mathbf{x}_{t i}\right) \rho\left(\mathbf{y}_{t, t-1} ; \theta_{0}\right) f_{k_{t}, k_{t-1} \mid \mathbf{s}_{t i}} d k_{t} d k_{t-1}+o_{p}\left(n^{-1 / 2}\right) .
\end{aligned}
$$

Proof. (a) Note that uniformly over $\tilde{\theta} \in \mathcal{N}_{02 n}$,

$$
\begin{aligned}
& \frac{d \widehat{Q}_{n}(\tilde{\theta})}{d \theta}\left[v_{2 n}^{*}\right]-\frac{1}{n} \sum_{i=1}^{n} \int\left\{\frac{d g\left(\mathbf{x}_{t i} ; \theta_{0}\right)}{d \theta}\left[v_{2}^{*}\right]\right\}^{\prime} A\left(\mathbf{x}_{t i}\right) \hat{g}\left(\mathbf{x}_{t i} ; \tilde{\theta}\right) f_{k_{t}, k_{t-1} \mid \mathbf{s}_{t i}} d k_{t} d k_{t-1} \\
= & \frac{1}{n} \sum_{i=1}^{n} \int\left[\left\{\frac{d \hat{g}\left(\mathbf{x}_{t i} ; \theta\right)}{d \theta}\left[v_{2 n}^{*}\right]-\frac{d g\left(\mathbf{x}_{t i} ; \theta_{0}\right)}{d \theta}\left[v_{2 n}^{*}\right]\right\}^{\prime} \hat{A}\left(\mathbf{x}_{t i}\right) \hat{g}\left(\mathbf{x}_{t i} ; \tilde{\theta}\right) \hat{f}_{k_{t}, k_{t-1} \mid \mathbf{s}_{t i}}\right. \\
+ & \left.+\frac{d g\left(\mathbf{x}_{t i} ; \theta_{0}\right)}{d \theta}\left[v_{2 n}^{*}\right]\right\}^{\prime}\left\{\hat{A}\left(\mathbf{x}_{t i}\right)-A\left(\mathbf{x}_{t i}\right)\right\} \hat{g}\left(\mathbf{x}_{t i} ; \tilde{\theta}\right) \hat{f}_{k_{t}, k_{t-1} \mid \mathbf{s}_{t i}} \\
& +\left\{\frac{d g\left(\mathbf{x}_{t i} ; \theta_{0}\right)}{d \theta}\left[v_{2 n}^{*}\right]\right\}^{\prime} A\left(\mathbf{x}_{t i}\right) \hat{g}\left(\mathbf{x}_{t i} ; \tilde{\theta}\right)\left\{\hat{f}_{k_{t}, k_{t-1} \mid \mathbf{s}_{t i}}-f_{\left.k_{t}, k_{t-1} \mid \mathbf{s}_{t i}\right\}}\right\} \\
& +\left\{\frac{d g\left(\mathbf{x}_{t i} ; \theta_{0}\right)}{d \theta}\left[v_{2 n}^{*}-v_{2}^{*}\right]\right\}^{\prime} A\left(\mathbf{x}_{t i}\right) \hat{g}\left(\mathbf{x}_{t i} ; \tilde{\theta}\right) f_{\left.k_{t}, k_{t-1} \mid \mathbf{s}_{t i}\right] d k_{t} d k_{t-1}} \\
\equiv & D_{1}+D_{2}+D_{3}+D_{4} .
\end{aligned}
$$


Also note that from the proof of Lemma C.3 (a)

$$
\sup _{\tilde{\theta} \in \mathcal{N}_{02 n}} \frac{1}{n} \sum_{i=1}^{n}\left\|\frac{d \hat{g}\left(\mathbf{x}_{t i} ; \theta\right)}{d \theta}\left[v_{2 n}^{*}\right]-\frac{d g\left(\mathbf{x}_{t i} ; \theta\right)}{d \theta}\left[v_{2 n}^{*}\right]\right\|_{E}^{2}=o_{p}\left(n^{-1 / 2}\right)
$$

and from Corollary C.1 (ii) in Ai and Chen (2003)

$$
\sup _{\tilde{\theta} \in \mathcal{N}_{02 n}} \frac{1}{n} \sum_{i=1}^{n}\left\|\frac{d \hat{g}\left(\mathbf{x}_{t i} ; \theta\right)}{d \theta}\left[v_{2 n}^{*}\right]-\frac{d g\left(\mathbf{x}_{t i} ; \theta\right)}{d \theta}\left[v_{2 n}^{*}\right]\right\|_{E}^{2}=o_{p}\left(n^{-1 / 2}\right) .
$$

Thus $D_{1}=o_{p}\left(n^{-1 / 2}\right)$ by Lemma C.2 (b) and Assumption 5.6. $D_{2}=o_{p}\left(n^{-1 / 2}\right)$ by Assumption 5.15 and Lemma C.2 (b). $D_{3}=o_{p}\left(n^{-1 / 2}\right)$ by $\left\|\hat{\alpha}-\alpha_{0}\right\|_{\alpha}=o_{p}\left(n^{-1 / 4}\right)$ and Lemma C.2 (b). $D_{4}=o_{p}\left(n^{-1 / 2}\right)$ by Assumption 6.7 and Lemma C.2 (b). Thus the result follows.

(b) Define $\varphi\left(\mathbf{x}_{t}, v_{2}^{*}\right)=\left\{\frac{d g\left(\mathbf{x}_{t} ; \theta_{0}\right)}{d \theta}\left[v_{2}^{*}\right]\right\}^{\prime} A\left(\mathbf{x}_{t}\right)$ and

$$
\mathcal{F}=\left\{\varphi\left(\mathbf{x}_{t}, v_{2}^{*}\right) \tilde{g}\left(\mathbf{x}_{t} ; \theta\right): \theta \in \mathcal{N}_{02 n}, \tilde{g} \in \Lambda_{c}^{\gamma}\left(\mathcal{X}_{t}\right) \text { s.t. } \sup _{\mathbf{x}_{t} \in \mathcal{X}_{t}, \theta \in \mathcal{N}_{02 n}}\left|\tilde{g}\left(\mathbf{x}_{t} ; \theta\right)-g\left(\mathbf{x}_{t} ; \theta\right)\right|=o(1)\right\} .
$$

By the argument of Corollary C.3 (ii) in Ai and Chen (2003), $\mathcal{F}$ is a Donsker class. We also note that $E\left[\left\|\varphi\left(\mathbf{x}_{t i}, v_{2}^{*}\right)\left\{\hat{g}\left(\mathbf{x}_{t i} ; \theta\right)-g\left(\mathbf{x}_{t i} ; \theta\right)\right\}\right\|^{2}\right]=o_{p}(1)$ and $\hat{g}\left(\mathbf{x}_{t i} ; \theta\right) \in \Lambda_{c}^{\gamma}\left(\mathcal{X}_{t}\right)$ uniformly over $\theta \in \mathcal{N}_{02 n}$ by Assumptions 5.1, 5.3, 5.4, 5.7, 5.18. Thus we obtain uniformly over $\theta \in \mathcal{N}_{02 n}$,

$$
\begin{array}{r}
\frac{1}{n} \sum_{i=1}^{n} \varphi\left(\mathbf{x}_{t i}, v_{2}^{*}\right)\left\{\hat{g}\left(\mathbf{x}_{t i} ; \theta\right)-g\left(\mathbf{x}_{t i} ; \theta\right)\right\}-E\left[\varphi\left(\mathbf{x}_{t i}, v_{2}^{*}\right)\left\{\hat{g}\left(\mathbf{x}_{t i} ; \theta\right)-g\left(\mathbf{x}_{t i} ; \theta\right)\right\}\right]=o_{p}\left(n^{-1 / 2}\right)(15) \\
\frac{1}{n} \sum_{i=1}^{n} \varphi\left(\mathbf{x}_{t i}, v_{2}^{*}\right)\left\{\hat{g}\left(\mathbf{x}_{t i} ; \theta_{0}\right)-g\left(\mathbf{x}_{t i} ; \theta_{0}\right)\right\}-E\left[\varphi\left(\mathbf{x}_{t i}, v_{2}^{*}\right)\left\{\hat{g}\left(\mathbf{x}_{t i} ; \theta_{0}\right)-g\left(\mathbf{x}_{t i} ; \theta_{0}\right)\right\}\right]=o_{p}\left(n^{-1 / 2}\right)(16)
\end{array}
$$

by Lemma 1 of Chen, Linton, and van Keilegom (2003). Combining (15) and (16) we obtain (because $\hat{\theta} \in \mathcal{N}_{02 n}$ )

$$
\begin{aligned}
& \frac{1}{n} \sum_{i=1}^{n} \varphi\left(\mathbf{x}_{t i}, v_{2}^{*}\right)\left\{\hat{g}\left(\mathbf{x}_{t i} ; \hat{\theta}\right)-\hat{g}\left(\mathbf{x}_{t i} ; \theta_{0}\right)\right\}-\frac{1}{n} \sum_{i=1}^{n} \varphi\left(\mathbf{x}_{t i}, v_{2}^{*}\right)\left\{g\left(\mathbf{x}_{t i} ; \hat{\theta}\right)-g\left(\mathbf{x}_{t i} ; \theta_{0}\right)\right\} \\
= & E\left[\varphi\left(\mathbf{x}_{t i}, v_{2}^{*}\right)\left\{\hat{g}\left(\mathbf{x}_{t i} ; \hat{\theta}\right)-\hat{g}\left(\mathbf{x}_{t i} ; \theta_{0}\right)\right\}\right]-E\left[\varphi\left(\mathbf{x}_{t i}, v_{2}^{*}\right)\left\{g\left(\mathbf{x}_{t i} ; \hat{\theta}\right)-g\left(\mathbf{x}_{t i} ; \theta_{0}\right)\right\}\right]+o_{p}\left(n^{-1 / 2}\right) .
\end{aligned}
$$

Now define $\hat{\varphi}\left(\mathbf{x}_{t}, v_{2}^{*}\right)=\int \varphi\left(\mathbf{x}_{t}, v_{2}^{*}\right) \hat{f}_{\mathbf{y}_{t, t-1} \mid \mathbf{x}_{t}} d \mathbf{y}_{t, t-1}$. We then obtain

$$
\begin{aligned}
E\left[\varphi\left(\mathbf{x}_{t i}, v_{2}^{*}\right)\left\{\hat{g}\left(\mathbf{x}_{t i} ; \hat{\theta}\right)-\hat{g}\left(\mathbf{x}_{t i} ; \theta_{0}\right)\right\}\right] & =E\left[\varphi\left(\mathbf{x}_{t i}, v_{2}^{*}\right) \int\left\{\rho\left(\mathbf{y}_{t, t-1} ; \hat{\theta}\right)-\rho\left(\mathbf{y}_{t, t-1} ; \theta_{0}\right)\right\} \hat{f}_{\mathbf{y}_{t, t-1} \mid \mathbf{x}_{t i}} d \mathbf{y}_{t, t-1}\right] \\
& =E\left[\int \varphi\left(\mathbf{x}_{t i}, v_{2}^{*}\right) \hat{f}_{\mathbf{y}_{t, t-1} \mid \mathbf{x}_{t i}} d \mathbf{y}_{t, t-1} E\left[\rho\left(\mathbf{y}_{t, t-1} ; \hat{\theta}\right)-\rho\left(\mathbf{y}_{t, t-1} ; \theta_{0}\right) \mid \mathbf{x}_{t i}\right]\right] \\
& =E\left[\hat{\varphi}\left(\mathbf{x}_{t i}, v_{2}^{*}\right)\left\{g\left(\mathbf{x}_{t i} ; \hat{\theta}\right)-g\left(\mathbf{x}_{t i} ; \theta_{0}\right)\right\}\right]
\end{aligned}
$$


and

$$
\begin{aligned}
& E\left[\hat{\varphi}\left(\mathbf{x}_{t i}, v_{2}^{*}\right)\left\{g\left(\mathbf{x}_{t i} ; \hat{\theta}\right)-g\left(\mathbf{x}_{t i} ; \theta_{0}\right)\right\}\right]-E\left[\varphi\left(\mathbf{x}_{t i}, v_{2}^{*}\right)\left\{g\left(\mathbf{x}_{t i} ; \hat{\theta}\right)-g\left(\mathbf{x}_{t i} ; \theta_{0}\right)\right\}\right] \\
= & E\left[\left\{\hat{\varphi}\left(\mathbf{x}_{t i}, v_{2}^{*}\right)-\varphi\left(\mathbf{x}_{t i}, v_{2}^{*}\right)\right\}\left\{g\left(\mathbf{x}_{t i} ; \hat{\theta}\right)-g\left(\mathbf{x}_{t i} ; \theta_{0}\right)\right\}\right]=o_{p}\left(n^{-1 / 2}\right) .
\end{aligned}
$$

It follows that

$$
\frac{1}{n} \sum_{i=1}^{n} \varphi\left(\mathbf{x}_{t i}, v_{2}^{*}\right)\left\{\hat{g}\left(\mathbf{x}_{t i} ; \hat{\theta}\right)-\hat{g}\left(\mathbf{x}_{t i} ; \theta_{0}\right)\right\}-\frac{1}{n} \sum_{i=1}^{n} \varphi\left(\mathbf{x}_{t i}, v_{2}^{*}\right)\left\{g\left(\mathbf{x}_{t i} ; \hat{\theta}\right)-g\left(\mathbf{x}_{t i} ; \theta_{0}\right)\right\}=o_{p}\left(n^{-1 / 2}\right) .
$$

Then for some $\bar{\theta} \in \mathcal{N}_{02 n}$, which lies between $\hat{\theta}$ and $\theta_{0}$, the mean value theorem (applied in the third equality) provides

$$
\begin{aligned}
& \frac{1}{n} \sum_{i=1}^{n} \varphi\left(\mathbf{x}_{t i}, v_{2}^{*}\right)\left\{\hat{g}\left(\mathbf{x}_{t i} ; \hat{\theta}\right)-\hat{g}\left(\mathbf{x}_{t i} ; \theta_{0}\right)\right\} \\
= & \frac{1}{n} \sum_{i=1}^{n} \varphi\left(\mathbf{x}_{t i}, v_{2}^{*}\right)\left\{g\left(\mathbf{x}_{t i} ; \hat{\theta}\right)-g\left(\mathbf{x}_{t i} ; \theta_{0}\right)\right\}+o_{p}\left(n^{-1 / 2}\right) \\
= & E\left[\varphi\left(\mathbf{x}_{t i}, v_{2}^{*}\right)\left\{g\left(\mathbf{x}_{t i} ; \hat{\theta}\right)-g\left(\mathbf{x}_{t i} ; \theta_{0}\right)\right\}\right]+o_{p}\left(n^{-1 / 2}\right) \\
= & \left\langle v_{2}^{*}, \hat{\theta}-\theta_{0}\right\rangle_{\theta}+E\left[\varphi\left(\mathbf{x}_{t i}, v_{2}^{*}\right)\left(\frac{d g\left(\mathbf{x}_{t i} ; \bar{\theta}\right)}{d \theta}\left[\hat{\theta}-\theta_{0}\right]-\frac{d g\left(\mathbf{x}_{t i} ; \theta_{0}\right)}{d \theta}\left[\hat{\theta}-\theta_{0}\right]\right)\right]+o_{p}\left(n^{-1 / 2}\right) \\
= & \left\langle v_{2}^{*}, \hat{\theta}-\theta_{0}\right\rangle_{\theta}+o_{p}\left(n^{-1 / 2}\right)
\end{aligned}
$$

where the second equality holds because the class of functions $\left\{\varphi\left(\mathbf{x}_{t i}, v_{2}^{*}\right)\left\{g\left(\mathbf{x}_{t i} ; \theta\right)-g\left(\mathbf{x}_{t i} ; \theta_{0}\right)\right\}\right.$ : $\left.\theta \in \mathcal{N}_{02 n}\right\}$ is a Donsker class and the last result holds by Assumption 6.10. Thus the result follows.

(c) By the definition of $\hat{\varphi}\left(\mathbf{x}_{t}, v_{2}^{*}\right)$, we obtain

$$
\begin{aligned}
& \frac{1}{n} \sum_{i=1}^{n} \int \varphi\left(\mathbf{x}_{t i}, v_{2}^{*}\right)\left\{\hat{g}\left(\mathbf{x}_{t i} ; \theta_{0}\right)-\rho\left(\mathbf{y}_{t, t-1} ; \theta_{0}\right)\right\} f_{k_{t}, k_{t-1} \mid \mathbf{s}_{t i}} d k_{t} d k_{t-1} \\
= & \frac{1}{n} \sum_{i=1}^{n} \int\left\{\hat{\varphi}\left(\mathbf{x}_{t i}, v_{2}^{*}\right)-\varphi\left(\mathbf{x}_{t i}, v_{2}^{*}\right)\right\} \rho\left(\mathbf{y}_{t, t-1} ; \theta_{0}\right) f_{k_{t}, k_{t-1} \mid \mathbf{s}_{t i}} d k_{t} d k_{t-1} .
\end{aligned}
$$

Thus the result follows by the Markov inequality since $\hat{\varphi}\left(\mathbf{x}_{t i}, v_{2}^{*}\right)-\varphi\left(\mathbf{x}_{t i}, v_{2}^{*}\right)=o_{p}(1)$ uniformly over $\mathbf{x}_{t i} \in \mathcal{X}_{t}$ and $g\left(\mathbf{x}_{t i} ; \theta_{0}\right)=0$, as in Ai and Chen (2003).

We now prove Theorem 6.12.

Proof. 1. The result follows from a similar argument to the proof of Theorem 3 in $\mathrm{Hu}$ and Schennach 
(2008). In the proof the bound for the pathwise second derivative is obtained by

$$
\begin{aligned}
& \left|\sup _{\alpha \in \mathcal{N}_{02 n}} \frac{d^{2}}{d \alpha d \alpha} \ln f_{r_{t}, k_{t}^{*}, k_{t-1}^{*} \mid \mathbf{z}_{t}^{*}}\left(r_{t}, k_{t}^{*}, k_{t-1}^{*} \mid \mathbf{z}_{t}^{*} ; \bar{\alpha}\right)\left[v_{1 n},\left(\alpha-\alpha_{0}\right)\right]\right| \\
\leq & \sup _{\alpha \in \mathcal{N}_{02 n}}\left[\left|\frac{f_{r_{t}, k_{t}^{*}, k_{t-1}^{*} \mid \mathbf{z}_{t}^{*}}^{|1|}\left(r_{t}, k_{t}^{*}, k_{t-1}^{*} \mid \mathbf{z}_{t}^{*} ; \bar{\alpha}, \nu\right)}{f_{r_{t}, k_{t}^{*}, k_{t-1}^{*} \mid \mathbf{z}_{t}^{*}}\left(r_{t}, k_{t}^{*}, k_{t-1}^{*} \mid \mathbf{z}_{t}^{*} ; \bar{\alpha}\right)}\right|^{2}+\left|\frac{\left.f_{r_{t}, k_{t}^{*}, k_{t-1}^{*} \mid \mathbf{z}_{t}^{*}}^{|2|} \cdot \mid \mathbf{z}_{t}^{*} ; \bar{\alpha}, \nu\right)}{f_{r_{t}, k_{t}^{*}, k_{t-1}^{*} \mid \mathbf{z}_{t}^{*}}\left(\cdot \mid \mathbf{z}_{t}^{*} ; \bar{\alpha}\right)}\right|\right]\left\|\alpha-\alpha_{0}\right\|_{s, \alpha}\left\|v_{1 n}\right\|_{s, \alpha}
\end{aligned}
$$

where $f_{r_{t}, k_{t}^{*}, k_{t-1}^{*} \mid \mathbf{z}_{t}^{*}}^{|2|}\left(r_{t}, k_{t}^{*}, k_{t-1}^{*} \mid \mathbf{z}_{t}^{*} ; \bar{\alpha}, \nu\right)$ is defined as $\left.\frac{d^{2}}{d \tau^{2}} f_{r_{t}, k_{t}^{*}, k_{t-1}^{*} \mid \mathbf{z}_{t}^{*}}\left(r_{t}, k_{t}^{*}, k_{t-1}^{*} \mid \mathbf{z}_{t}^{*} ; \bar{\alpha}+\tau \nu^{-1}\right)\right|_{\tau=0}$ with absolute values of $\bar{f}_{1}, \bar{f}_{2}, \frac{d}{d \psi} f_{r_{t} \mid \mathbf{z}_{t}}$, and $\frac{d^{2}}{d \psi^{2}} f_{r_{t} \mid \mathbf{z}_{t}}$.

2. Let $\varepsilon_{n}=o\left(n^{-1 / 2}\right)>0$ and $u_{2 n}^{*}= \pm v_{2 n}^{*}$. By a Tayor expansion around $\hat{\theta}$, we obtain that for $\theta_{s} \in\left(\hat{\theta}, \hat{\theta}+\varepsilon_{n} u_{2 n}^{*}\right)$,

$$
\begin{aligned}
& \widehat{Q}_{n}(\hat{\theta})-\widehat{Q}_{n}\left(\hat{\theta}+\varepsilon_{n} u_{2 n}^{*}\right) \\
= & \frac{d \widehat{Q}_{n}(\hat{\theta})}{d \theta}\left[\varepsilon_{n} u_{2 n}^{*}\right]+\frac{d^{2} \widehat{Q}_{1 n}\left(\theta_{s}\right)}{d \theta d \theta}\left[\varepsilon_{n} u_{2 n}^{*}, \varepsilon_{n} u_{2 n}^{*}\right]+\frac{d^{2} \widehat{Q}_{2 n}\left(\theta_{s}\right)}{d \theta d \theta}\left[\varepsilon_{n} u_{2 n}^{*}, \varepsilon_{n} u_{2 n}^{*}\right] .
\end{aligned}
$$

Then by Lemma C.3 and Lemma C.4, we have

$$
\sqrt{n}\left\langle v_{2 n}^{*}, \hat{\theta}-\theta_{0}\right\rangle_{\theta}=-\frac{1}{\sqrt{n}} \sum_{i=1}^{n} \int\left\{\frac{d g\left(\mathbf{x}_{t i} ; \theta_{0}\right)}{d \theta}\left[v_{2 n}^{*}\right]\right\}^{\prime} A\left(\mathbf{x}_{t i}\right) \rho\left(\mathbf{y}_{t, t-1} ; \theta_{0}\right) f_{k_{t}, k_{t-1} \mid \mathbf{s}_{t i}} d k_{t} d k_{t-1}+o_{p}(1) .
$$

Thus the result follows by the Lindeberg-Lévy central limit theorem. 


\section{References}

[1] Ackerberg, D., K. Caves, and G. Frazer (2006), "Structural Identification of Production Functions," working paper.

[2] Ai, C. and X. Chen (2003), "Efficient Estimation of Models with Conditional Moment Restrictions Containing Unknown Functions," Econometrica, 71, 1795-1843.

[3] Andrews, Donald W. K. (2011), "Examples of L2-Complete and Boundedly-Complete Distributions," working paper.

[4] Blundell, R., X. Chen, and D. Kristensen (2007), "Semi-nonparametric IV Estimation of Shape-invariant Engel Curves," Econometrica, 75, 1613-1669.

[5] Blundell, R. and S. Bond (2000), "GMM Estimation with Persistent Panel Data: An Application to Production Functions," Econometric Reviews, 19, 321-340.

[6] Chen, X. (2007), "Large Sample Sieve Estimation of Semi-Nonparametric Models," in J. J. Heckman and E. Leamer (eds.), Handbook of Econometrics, 6B, 5549-5632. Amsterdam: Elsevier.

[7] Chen, X., O. Linton, and I. van Keilegom (2003), "Estimation of Semiparametric Models when the Criterion Function is not Smooth," Econometrica, 71, 1591-1608.

[8] Darolles, S., Y. Fan, J. Florens, and E. Renault (2011), "Non-parametric Instrumental Regression," Econometrica, 79, 1541-1565.

[9] Griliches,Z. and J. Mairesse (1998), "Production Functions: The Search for Identification," in S. Strøm (ed.), The Ragnar Frisch Centennial Symposium, Cambridge University Press, 169-203.

[10] Hall, P. and J. Horowitz (2005), "Nonparametric Methods for Inference in the Presence of Instrumental Variables," Annals of Statistics, 33, 2904-2929.

[11] Horowitz, J. (2006), "Testing a Parametric Model Against a Nonparametric Alternative with Identification Through Instrumental Variables," Econometrica, 74, 521-538.

[12] Huang, G. and Y. Hu (2011), "Estimating Production Functions with Robustness Against Errors in Proxy Variables," working paper.

[13] Hu, Y and S. Schennach (2008), "Instrumental Variable Treatment of Nonclassical Measurement Error Models," Econometrica, 76, 195-216.

[14] Levinsohn, J. and A. Petrin (2003), "Estimating Production Functions Using Inputs to Control for Unobservables," Review of Economic Studies, 70, 317-342. 
[15] Marschak, J. and W. Andrews (1944), "Random Simultaneous Equations and the Theory of Production," Econometrica, 12, 143-205.

[16] Newey, W., and J. Powell (2003), "Instrumental Variable Estimation of Nonparametric Models," Econometrica, 71, 1565-1578.

[17] Robinson, P. (1988), "Root-n Consistent Semiparametric Regression," Econometrica, $55,931-954$.

[18] Olley, S. and A. Pakes (1996), "The Dynamics of Productivity in the Telecommunications Equipment Industry," Econometrica, 64, 1263-1298.

[19] Song, S. (2012), "Semiparametric Estimation of Models with Conditional Moment Restrictions in the Presence of Nonclassical Measurement Errors," working paper.

[20] Wooldridge, J. (2005), "On Estimating Firm-level Production Functions Using Proxy Variables to Control for Unobservables," working paper. 